Portland State University

PDXScholar

Fall 12-5-2016

\title{
Investigations into the Effects of Water Exchange and the Structure of Lanthanide Chelates
}

Katherine Marie Payne

Portland State University

Follow this and additional works at: https://pdxscholar.library.pdx.edu/open_access_etds

Part of the Chemistry Commons, and the Diagnosis Commons

Let us know how access to this document benefits you.

\section{Recommended Citation}

Payne, Katherine Marie, "Investigations into the Effects of Water Exchange and the Structure of Lanthanide Chelates" (2016). Dissertations and Theses. Paper 3284.

https://doi.org/10.15760/etd.3275

This Dissertation is brought to you for free and open access. It has been accepted for inclusion in Dissertations and Theses by an authorized administrator of PDXScholar. Please contact us if we can make this document more accessible: pdxscholar@pdx.edu. 
Investigations into the Effects of Water Exchange and the

Structure of Lanthanide Chelates

by

Katherine Marie Payne

A dissertation submitted in partial fulfillment of the requirements for the degree of

\author{
Doctor of Philosophy \\ in \\ Chemistry
}

\title{
Dissertation Committee: \\ Mark Woods, Chair \\ David R. Stuart \\ Robert M. Strongin \\ Jon J. Abramson
}

Portland State University

2016 
(C) 2016 Katherine Marie Payne 


\begin{abstract}
Lanthanide chelates are effective agents for improving contrast in MR images. Optimizing the relaxation of inner sphere water molecules is a common focus of research in this field. However, the efforts to design an optimal contrast agent have commonly over-looked the relationship of water position and water exchange kinetics. This work explores structural conformation, the impact of very fast water exchange kinetics on hydration, and differing tumbling rates for regioisomers of a number of lanthanide chelates. We have grown crystals of LnDOTMA and obtained structural data by X-ray diffraction that provide a picture of the chelate during water exchange and demonstrate that chelate conformation is associated with water position. We observe increased population of the major isomer with increased water exchange rates in variable temperature ${ }^{1} \mathrm{H}$ NMR studies of HoDOTMA. This suggests that water position and water exchange rates are linked. We therefore recommend that accurate water exchange data be included in the application of the SBM equations when interpreting experimental data. As further support of this recommendation, we measured water exchange kinetics with ${ }^{17} \mathrm{O}$ NMR for the rigid GdNB-DOTMA chelates. These results were used in the fitting of ${ }^{1} \mathrm{H}$ NMRD profiles to establish tumbling parameters. Similar results were also observed in the less rigid GdNB-DOTA, establishing the first identification of regioisomers in these chelates and their biphenyl derivatives. Binding studies of GdBP-DOTA indicate that the side isomer is a more effective agent, but it is the minor species in solution. Our work herein shows that predicting efficacy of contrast agents with SBM equations requires a more complete consideration of chelate hydration $\left(q / r^{6}\right)$.
\end{abstract}




\section{Acknowledgments}

I would like to express my gratitude for those who supported my endeavor for a Ph.D.:

Prof. Mark Woods for his enthusiasm for great science and educating students. Also for his vision behind the science described herein and for his continued encouragement to not waste potential.

Dr. Benjamin Webber, Lauren Rust, Jacqueline Slack, Guadalupe Camacho, Annah Farashishiko, Joseph Armstrong, Jennifer Wilds-Montgomery, Karley Maier, and Christina Miller for great science discussion, assistance of all kinds, listening to endless practice talks, critical feedback, and general development of a great office environment.

Prof. Mauro Botta for graciously hosting me and providing access to his laboratory facilities for relaxometric data acquisition, for many discussions with Mark and myself concerning NMRD profiles, ${ }^{17} \mathrm{O}$ NMR data, and hydration states, and for his invaluable insight into interpreting the aforementioned data.

Professors David Stuart, Robert Strongin, and Jon Abramson for their time, critical feedback, and support at various stages during the process of acquiring a Ph.D.

Prof. David Peyton, Dr. Robert Jensen, and Dr. Andrea DeBarber for their assistance in acquiring and interpreting various spectral data. 
Prof. Kevin Reynolds, Prof. Andrea Goforth, Prof. Dirk Iwata-Reuyl, and various members of their respective groups for generously allowing use of their laboratory facilities and instrumentation.

Prof. Edward Valente and Prof. Theresa McCormick for their thoughtful collaborations and educational guidance.

Prof. Gwen Shusterman and Prof. Eric Sheagley for their mentoring advice and educational discussions, as well as their general feedback and support.

My family, the Yeates family, and many other close friends for their overall support, patience, and sympathetic ears throughout my graduate education - without them my perseverance would have been insufficient.

Portland State University, Oregon Health \& Sciences University, National Institutes of Health, National Science Foundation, and Piedmonte University for financial support.

Finally, to the many members of the Department of Chemistry - particularly the incoming class of Fall 2010 - who provided camaraderie, collaborations, and fostered a welcoming science environment over the years. Thank you. 


\section{Table of Contents}

Abstract

$\begin{array}{ll}\text { Acknowledgments } & \text { ii }\end{array}$

List of Tables $\quad$ vii

List of Figures $\quad$ viii

List of Equations $\quad$ xii

Declaration $\quad$ xiii

CHAPTER 1. Introduction to lanthanide chelates and how they are employed to enhance

MRI contrast

1.1 General information 1

1.2 MR signal and relaxation 2

1.3 Paramagnetic contrast agents 4

1.4 Solomon Bloembergen Morgan Theory 6

1.4.1 Defining SBM Equations 6

1.4.2 Narrowing down the SBM parameters $\quad 8$

1.4.3 Relaxometric analysis of contrast agents 11

$\begin{array}{ll}1.5 \text { Contrast agent ligand design } & 13\end{array}$

1.6 Isomerism in LnDOTA chelates 16

1.7 Preventing helicity inversion in LnDOTA-type chelates 21

1.8 Hydration of the geometric isomers and its effect on relaxivity 23

1.9 Using other Ln3+ ions for probing GdDOTA-type systems 26

$\begin{array}{ll}1.10 \text { This work } & 27\end{array}$ 
CHAPTER 2. Structural variations in the crystal structures of DOTMA chelates across the lanthanide series may reflect conformational changes associated with water exchange in solution

2.1 Crystal structures: a window into solution state behavior

2.2 A survey of crystallographic data of LnDOTA-type chelates

2.3 Structural variation in LnDOTMA crystals

2.4 The early LnDOTMA series

2.5 The late LnDOTMA series

2.6 The complete LnDOTMA series

2.6.1 Considering the series as a whole

2.6.2 Other structural changes associated with hydration

2.7 Crystal structure and solution state of HoDOTMA

2.8 Conclusions

CHAPTER 3. Investigations into the relaxometric properties of various isomers of

3.1 Background

3.2 Determination of $\tau_{M}$ in the isomers of GdNB-DOTMA

$3.3{ }^{1} \mathrm{H}$ Relaxometric studies of the isomers of the GdNB-DOTMA

CHAPTER 4. Effects of regioisomerism in the LnNB-DOTA system 
4.2 Synthesis and purification of chelates: does regioisomerism occur in the LnNB-DOTA system?

4.3 Structural isomers of LnNB-DOTA

4.3.1 Isomer identification

4.3.2 Orientation of the benzylic group

4.4 Interconversion of regioisomers

4.5 GdNB-DOTA: How does regioisomerism effect NB-DOTA as a contrast agent?

4.6 Slowing rotation of GdBP-DOTA

4.6.1 GdBP-DOTA: can regioisomerism influence chelate binding?

4.6.2 Self-association of the biphenyl moiety

4.6.3 Biphenyl moiety interaction with poly- $\beta$-cyclodextrin

CHAPTER 5. Experimental

5.1 Synthesis of thesis complexes 


\section{List of Tables}

Table $1.1 K_{\text {GdL }}$ values for $\mathrm{Gd}^{3+}$ chelates of DOTA, DOTMA, and NB-DOTA

Table 1.2 $\tau_{\mathrm{M}}$ and $r_{1}$ values for GdNB-DO3A-1A biphenyl conjugates bound to $\beta$-CD 25

Table 2.1 Select parameters of the three $\mathrm{Y}^{3+}$ chelates from Lukeš and Hermann 33

Table 2.2 Select crystallographic parameters for LnDOTMA

Table 2.3 Select bond lengths and angles for LnDOTMA in the early series 51

Table 2.4 Select bond lengths and angles for all chelates in $\mathrm{Tb}^{3+}$ and DyDOTMA 56

Table 2.5 Select bond lengths and angles for $\mathrm{Ho}^{3+}, \mathrm{Er}^{3+}, \mathrm{Tm}^{3+}$, and YbDOTMA 57

Table 3.1 Fitting parameters for ${ }^{17} \mathrm{O}$ transverse relaxation data $\quad 84$

Table 3.2 Fitting parameters for ${ }^{1} \mathrm{H}$ NMRD profiles $\quad 87$

Table 4.1 Distribution of structural isomers in LnNB-DOTA crude solutions 98

Table 4.2 Distribution of geometric isomers in purified LnNB-DOTA solutions $\quad 100$

Table 4.3 Fitting parameters for ${ }^{1} \mathrm{H}$ NMRD profiles of the GdNB-DOTA isomers 117

Table 4.4 CMC and micelle size of GDBP-DOTA regioisomers 120

Table 4.5 Binding affinity constants for GdBP-DOTA regioisomers with poly- $\beta$-CD 124

Table 4.6 Fitting parameters for ${ }^{1} \mathrm{H}$ NMRD profiles of GdBP-DOTA regioisomers free and bound to poly- $\beta-\mathrm{CD}$

Table 5.1 Purification of LnNB-DOTMA chelates (from Ben Webber)

Table 5.2 Purification of LnNB-DOTA chelates

Table 5.3 Purification of LnBP-DOTA chelates 


\section{List of Figures}

Figure 1.1 Displaying 3

Figure 1.2 Interactions of $\mathrm{Ln}^{3+}$ chelates in solution 5

Figure 1.3 Connectivity of the GdDOTP chelate 9

Figure 1.4 The SBM curve showing the dependence of $r_{1}{ }^{\mathrm{IS}}$ on $\tau_{\mathrm{R}}$ and $\tau_{\mathrm{M}}$

Figure 1.5 An example of a typical NMRD profile for chelates with fast and slow $\tau_{\mathrm{R}} \quad 12$

Figure 1.6 Ligands of clinically approved contrast agents (DOTA and DTPA) 14

Figure 1.7 Crystal structure of EuDOTA 15

Figure 1.8 Interconversion of diastereoisomers in DOTA-type chelates 17

Figure $1.9{ }^{1} \mathrm{H}$ NMR spectrum of EuDOTA 18

Figure 1.10 Geometric isomer distribution in LnDOTA chelate solutions 19

Figure 1.11 Simulation of ${ }^{1} \mathrm{H}$ NMR spectrum for $\mathrm{YbDO} 3 \mathrm{AP}(\mathrm{ABn})$

Figure 1.12 The pro-S protons on the corner and side carbons of the [3333] ring 23

Figure 1.13 Connectivity of LnNB-DO3A-1A and its biphenyl conjugates 25

Figure 2.1 Connectivity structure of YBO3A-P(ABn) 30

Figure 2.2 Crystal structures of YBO3A-P $(\mathrm{ABn})$ complexes 31

Figure 2.3 Coordination cage of LnDOTA-type complexes 32

Figure 2.4 Connectivity structures of the LnDOTA-like chelates surveyed 35

Figure $2.5 \mathrm{~d} / \mathrm{c}$ ratios for the LnDOTA-type chelates surveyed 36

Figure 2.6 Crystal structures of $\mathrm{Eu}^{3+}, \mathrm{Gd}^{3+}$, and $\mathrm{Tb}^{3+}$ chelates of TCE-DOTA 39

Figure 2.7 Displaying the parallel polar sheets typical of the LnDOTMA crystals 41

Figure 2.8 Relationship of the side-centered orthorhombic and primitive cells 42 
Figure 2.9 Crystal packing of PrDOTMA

Figure 2.10 The additional crystal parameters used in our analysis

Figure 2.11 Chelates of the $\mathrm{Ce}^{3+}, \mathrm{Pr}^{3+}, \mathrm{Nd}^{3+}, \mathrm{Sm}^{3+}, \mathrm{Eu}^{3+}$, and GdDOTMA unit cells

Figure 2.12 Metal-water distances for crystals of the early $\mathrm{Ln}^{3+}$ chelates of DOTMA 48

Figure 2.13 Influence of ionic contraction in metal-donor atom distances 49

Figure $2.14 \mathrm{~d} / \mathrm{c}$ ratio for crystals of the early $\mathrm{Ln}^{3+}$ chelates of DOTMA 50

Figure 2.15 Chelates of the $\mathrm{Tb}^{3+}, \mathrm{Dy}^{3+}, \mathrm{Ho}^{3+}, \mathrm{Er}^{3+}, \mathrm{Tm}^{3+}$, and YbDOTMA unit cells 53

Figure 2.16 Metal-water distances for crystals of LnDOTMA

Figure 2.17 A cross-section of the LnDOTMA crystals 60

Figure 2.18 Relating $q$ to ionic contraction $\quad 61$

Figure 2.19 Relating $q / r^{6}$ to ionic contraction $\quad 62$

Figure $2.20 \mathrm{~d} / \mathrm{c}$ ratios for the LnDOTMA and the LnDOTA-type chelates surveyed 64

Figure 2.21 Relating the d/c ratio to metal-water distances 65

Figure 2.22 Relating the $\mathrm{O}_{4}$ area to ionic contraction 66

Figure 2.23 Relating coordination cage height to ionic contraction 67

Figure 2.24 Relating chelate torsion angles to ionic contraction $\quad 68$

Figure 2.25 Relating chelate torsion angles to metal-water distances for LnDOTMA 69

Figure 2.26 Relating chelate torsion angles to metal-water distances for LnDOTA-type

$\begin{array}{ll}\text { chelates surveyed } & 70\end{array}$

$\begin{array}{ll}\text { Figure 2.27 HoDOTMA crystal structure } & 71\end{array}$

Figure 2.28 Variable temperature ${ }^{1} \mathrm{H}$ NMR of HoDOTA and HoDOTMA 73

Figure 2.29 Variable temperature isomeric distribution of HoDOTA and HoDOTMA 74 
Figure $2.30{ }^{17} \mathrm{O}$ transverse relaxation and ${ }^{1} \mathrm{H}$ NMRD for $\mathrm{Ho}^{3+}$ chelates of DOTA, $\begin{array}{ll}\text { DOTMA, and TETA } & 76\end{array}$

Figure 3.1 Connectivity of the $S$-SSSS- and $S$-RRRR-LnNB-DOMTA chelates $\quad 81$

Figure $3.2{ }^{17} \mathrm{O}$ transverse relaxation of the regioisomers of $S-R R R R$ -

and S-SSSS-GdNB-DOTMA chelate $\quad 83$

Figure $3.3{ }^{1} \mathrm{H}$ NMRD profiles of the four isomers of GdNB-DOTMA 86

Figure 3.4 Significance of $\tau_{\text {off }}$ on hydration $\quad 90$

Figure 4.1 Connectivity of LnIB-DOTA and LnNB-DOTA chelates 95

Figure $4.2{ }^{1} \mathrm{H}$ NMR of the most shifted axial protons of crude EuNB-DOTA 96

Figure 4.3 HPLC chromatograms of $\mathrm{Yb}^{3+}$ and EuNB-DOTA 98

Figure $4.4{ }^{1} \mathrm{H}$ NMR of the most shifted axial protons of purified EuNB-DOTA

and YbNB-DOTA 99

Figure 4.5 Relative positions of the LnNB-DOTA chelate protons 101

Figure 4.6 ${ }^{1} \mathrm{H}-{ }^{1} \mathrm{H}$ COSY spectrum of the corner EuNB-DOTA isomer 103

Figure $4.7{ }^{1} \mathrm{H}-{ }^{1} \mathrm{H}$ COSY spectrum of the side EuNB-DOTA isomer 105

Figure 4.8 ${ }^{1} \mathrm{H}-{ }^{1} \mathrm{H}$ EXSY spectrum of the side EuNB-DOTA isomer 106

Figure 4.9 Simulated 1H NMR spectra for SAP and TSAP isomers of the side

$\begin{array}{ll}\text { EuNB-DOTA isomer } & 107\end{array}$

Figure $4.10{ }^{1} \mathrm{H}-{ }^{1} \mathrm{H}$ EXSY spectrum of the corner EuNB-DOTA isomer 108

Figure 4.11 VT ${ }^{1} \mathrm{H}$ NMR spectra of the most shifted axial protons of YbNB-DOTA 111

Figure 4.12 The whole bell curve possible from VT ${ }^{17} \mathrm{O}$ transverse relaxation plots 112

Figure $4.13{ }^{17} \mathrm{O}$ transverse relaxation of the regioisomers of GdNB-DOTA 113 
Figure $4.14{ }^{1} \mathrm{H}$ NMRD profiles of the GdNB-DOTA isomers 115

Figure 4.15 Titration of regioisomers of GdBP-DOTA to determine CMC 120

Figure $4.16{ }^{1} \mathrm{H}$ NMRD profiles of the GdBP-DOTA regioisomers, below and above the CMC

Figure 4.17 Titration of poly- $\beta$-CD into the regioisomers of GdBP-DOTA

Figure $4.18{ }^{1} \mathrm{H}$ NMRD profiles of the GdBP-DOTA regioisomers, free and bound to poly- $\beta-C D$

Figure 4.19 Benzylic region of ${ }^{1} \mathrm{H}$ NMR spectra of EuNB-DOTA regioisomers 


\section{List of Equations}

Equation 1.1 The Boltzmann distribution of spins 2

$\begin{array}{ll}\text { Equation 1.2 The observed longitudinal relaxivity } & 6\end{array}$

Equation 1.3 The inner sphere relaxation paramagnetic dependence $\quad 7$

Equation 1.4 The longitudinal relaxation time constant of a bound water proton 7

Equation 1.5 Defining the correlation time constant 8

Equation 3.1 Defining water exchange $\quad 89$ 


\section{Declaration}

The work herein was produced by the author between December 2010 and June 2016 in the Department of Chemistry at Portland State University, at the Advanced Imaging Research Center at Oregon Health \& Sciences University, and in the Department of Chemistry at Piedmonte, Italy. Work completed by collaborating researchers was accomplished in their laboratories at their respective institutes, as noted. All of this work is my own, unless otherwise stated, and no part of it has been submitted for a different degree at this or any other university. 


\section{CHAPTER 1: INTRODUCTION TO LANTHANIDE CHELATES AND HOW THEY ARE EMPLOYED TO ENHANCE MRI CONTRAST}

\subsection{General information}

Medical imaging was introduced over 120 years ago with the advent of X-ray technology. The ability to visualize anatomy without surgery has enabled great advances in medical diagnosis. Magnetic Resonance Imaging (MRI) has advantages over other imaging modalities: it is non-invasive, does not involve the use of ionizing radiation or radionuclides, and affords images of high spatial resolution. Since the first scan of a human wrist in $1977,{ }^{1}$ MRI has become one of the most commonly used techniques for obtaining physiological information.

Magnetic resonance of atomic nuclei was discovered by Rabi in the 1930s. ${ }^{2,3}$ The development of instrumentation to quantitatively analyze nuclear resonance was pioneered by Bloch and Purcell in the 1940s. ${ }^{4-8}$ The application of NMR theory to acquiring anatomical information via MRI started with the research of Damadian, Mansfield, and Lauterbur during the 1960s and into the 1970s. Damadian showed that the hydrogen signals in healthy and cancerous tissue are different; ${ }^{9}$ Mansfield developed mathematical analysis and advanced magnetic field gradients for improved imaging; ${ }^{10,11}$ and Lauterbur published the first 2D map of hydrogen distribution in a sample. ${ }^{12}$ Their competitive research led to the application of magnetic resonance from single nuclei signal detection to 2D and 3D anatomical images. Since the first human scan in 1977, MRI has been a fast growing field in clinical diagnosis. ${ }^{1,} 13$ For their discoveries 
contributing to the modern MRI many of these scientists were awarded Nobel prizes in 1944,1952 , and 2003.

\subsection{MR signal and relaxation}

Protons are the primary focus for MRI, as they are the most abundant nuclei in the body. MRI uses a strong magnet and radiofrequency radiation to probe the intrinsic nuclear spin ( $\mathrm{I}=1 / 2)$ of protons. MRI signal is generated much the same way as for NMR; both instruments utilize radiofrequency pulses to manipulate signal generated when nuclei with a magnetic moment are placed inside a strong magnet. When exposed to a magnetic field, nuclei in the sample with a magnetic moment will align to certain allowed axes with respect to the field, in accordance with the Zeeman effect (Figure 1.1A). For protons, this is either with $\left(\mathrm{N}_{\alpha}\right)$ or counter to $\left(\mathrm{N}_{\beta}\right)$ the external field $\left(\mathrm{B}_{0}\right)$. The distribution of protons between these relative populations is defined by the Boltzmann equation (Equation 1.1) and is dependent on $\mathrm{B}_{0}$, absolute temperature (T), the gyromagnetic constant $(\gamma)$, and Planck's and Boltzmann's constants ( $h$ and $k$ ).

$$
\frac{N_{\beta}}{N_{\alpha}}=e^{-B_{0} h \gamma / k T}
$$

\section{Equation 1.1: The Boltzmann distribution of spins}

The slight excess of protons found in the lower energy state creates a net magnetic moment aligned with $\mathrm{B}_{0}$. This small net magnetic moment is impossible to discern while parallel to $\mathrm{B}_{0}$; it must be rotated perpendicular to $\mathrm{B}_{0}$ to be detectible. This net magnetic moment has a constant oscillation related to the strength of $\mathrm{B}_{0}$. Radiofrequency pulses of appropriate quantum energy (related to the speed of oscillation for the net magnetic 
moment) are used to create a second magnetic field $\left(\mathrm{B}_{1}\right)$ perpendicular to $\mathrm{B}_{0}$ in order to manipulate the net magnetic moment into the detectible orientation (Figure 1.1B). After the net magnetic moment has been transferred into the perpendicular plane by $\mathrm{B}_{1}$ it then undergoes relaxation back to the Zeeman alignment. This relaxation occurs both by reestablishing the Boltzmann distribution ( $\mathrm{T}_{1}$ or longitudinal) and by losing coherence in the perpendicular frame ( $T_{2}$ or transverse), see Figure 1.1C. The time constants $T_{1}$ and $T_{2}$ (ms) describe these relaxation processes.

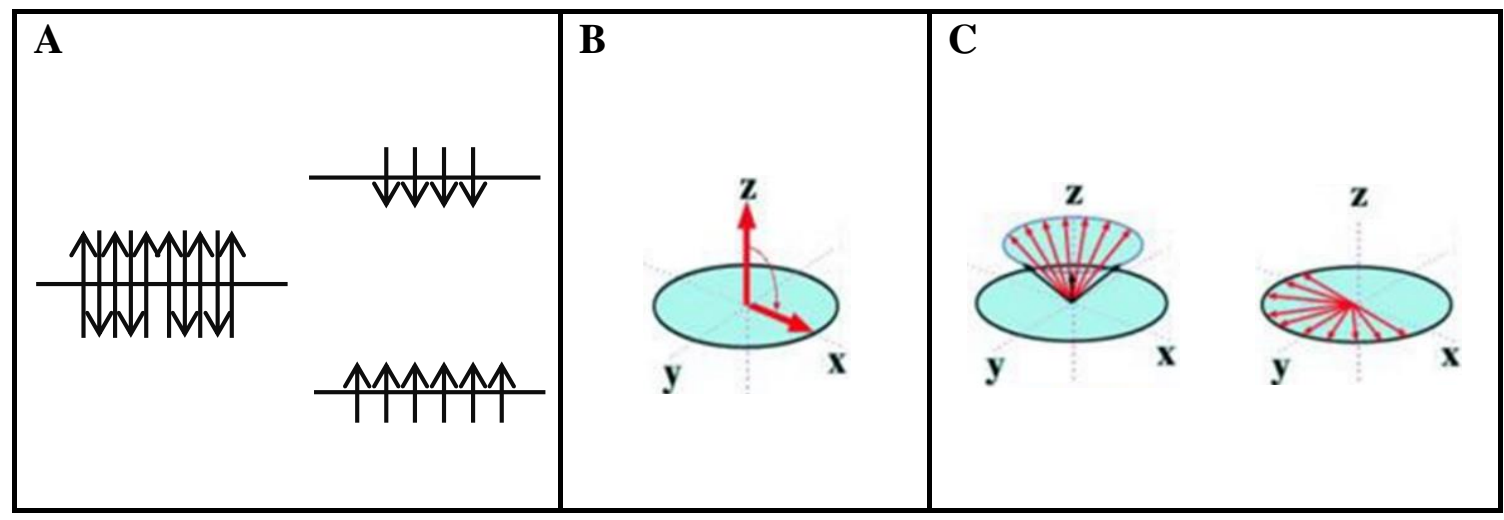

Figure 1.1: Displaying $(A)$ the effect of a strong $B_{0}$ field on degenerate protons, resulting in energy states populated according to the Boltzmann distribution, the effects of $(B)$ a $90^{\circ} B_{1}$ pulse on the net magnetic moment and $(C) T_{1}$ and $T_{2}$ relaxation on the ensemble of protons.

$\mathrm{T}_{1}$ refers to the time constant for returning $63 \%$ of the signal to the Boltzmann distribution; $\mathrm{T}_{2}$ refers to the time constant for $37 \%$ of the signal generated to decay as a result of coherence loss. These relaxation processes occur independently of each other to return the signal generated in the $\mathrm{B}_{1}$ plane back to its equilibrium alignment in $\mathrm{B}_{0}$. The relaxation time constants $\left(T_{1}\right.$ and $\left.T_{2}\right)$ of a proton depend on its freedom of rotation and the local magnetic field. To create an MR image the different relaxation time constants 
are assigned relative brightness - differences in the time constants in tandem with a gradient field $\left(\mathrm{B}_{1}\right)$ allow for this signal to be spatially located, generating a threedimensional image. Modulations in the local field of a proton can speed the relaxation process, shortening the time constants. This action can be facilitated by dipole-dipole interactions. Differences in relaxation time constants afford the contrast seen in MR images. To enhance this contrast and enable faster scans, MR images are commonly weighted to increase sensitivity for $\mathrm{T}_{1}$ or $\mathrm{T}_{2}$ by adjusting the time delays between various radiofrequency pulses.

\subsection{Paramagnetic contrast agents}

Before it was realized that ${ }^{1} \mathrm{H}$ NMR spectra of common organic molecules could be collected in a reasonable time frame, paramagnetic species were used to shorten $\mathrm{T}_{1}$ and $\mathrm{T}_{2}$ values. ${ }^{14-16}$ Referred to as paramagnetic catalysts in the early literature, these species are used to quickly establish thermal equilibrium to drastically shorten "inconveniently" long natural relaxation times. ${ }^{6}$ While it is now known that the transverse relaxation of protons occurs on a time-scale that does not require the presence of paramagnetic species for collection of ${ }^{1} \mathrm{H}$ NMR spectra, they are still routinely employed in MR to shorten relaxation time constants of protons proximal to the paramagnetic center - enabling the enhancement of contrast in anatomical images. Specifically, paramagnetic species with long electronic relaxation and anisotropic shells, such as $\mathrm{Gd}^{3+}$, shorten the spin relaxation of proximal nuclei, further enhancing contrast in MR images.

The functionality of contrast agents arises from dipole-dipole interactions between water protons and the unpaired electrons of the paramagnetic metal ion. The large dipole 
moments of the unpaired electrons of $\mathrm{Gd}^{3+}$ create a modulating local magnetic field, which catalyzes both the longitudinal and transverse relaxation of water protons. This results in increased contrast in $T_{1}$-weighted images between regions in which the contrast agent accumulates and those where it does not. The ability of a contrast agent to shorten the relaxation time constant of water protons (its effectiveness) is defined in terms of relaxivity, which has the units of $\mathrm{mM}^{-1} \mathrm{~s}^{-1}$.

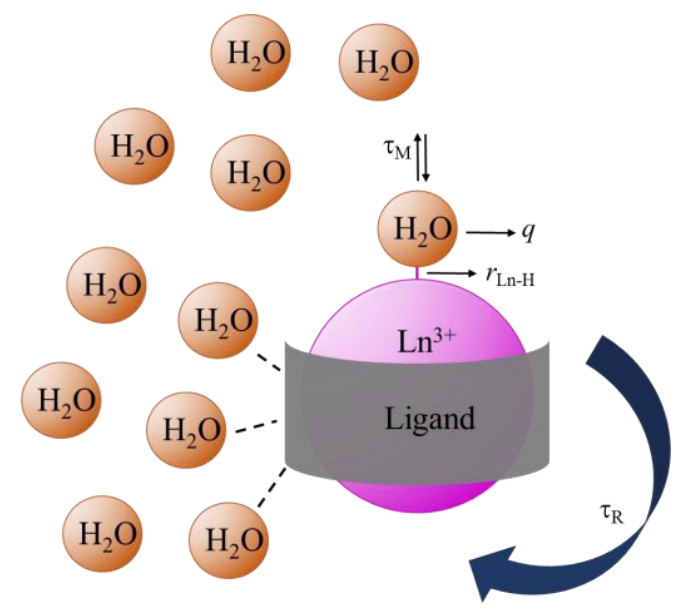

Figure 1.2: Interactions of $\mathrm{Ln}^{3+}$ chelates in solutions include water residence lifetime $\left(\tau_{\mathrm{M}}\right)$, distance to the inner sphere water $\left(\tau_{\llcorner\mathrm{LH}}\right)$, rotational correlation time $\left(\tau_{\mathrm{R}}\right)$.

The dipole-dipole interaction between the water proton and the paramagnetic ion is strongly distance dependent. For the $\mathrm{Ln}^{3+}$ chelates considered herein, there are three hydration spheres that describe the interaction of the chelate with water: the inner, second, and outer spheres (Figure 1.2). Both inner and secondary spheres have water exchanging between interacting with the contrast agent and being part of the bulk water in solution: inner sphere water is directly coordinated with the metal ion - the shortest distance of interaction; second sphere water is hydrogen-bonded to the ligand - typically 
a much longer distance than coordinated water. Outer sphere water is not in exchange with or coordinated to the contrast agent; this water is relaxed as it diffuses past the metal ion at a distance of over $4 \AA$ away. The segregation of inner, second, and outer sphere water as described above are simply qualifications commonly ascribed in our field, but there is no clear distinction between them in solution; water is free to move about where it will and, being a molecule, has no precise delineation of location relative to the contrast agent.

$$
\frac{1 / T_{1}^{o b s}-1 / T_{1 W}}{[\mathrm{Gd}]}=r_{1}=r_{1}^{I S}+r_{1}^{o S}+r_{1}^{S S}
$$

\section{Equation 1.2: The observed longitudinal relaxivity}

The observed relaxivity $\left(r_{1}\right)$ is the sum of relaxation in all the hydration spheres (Equation 1.2). The contribution from outer sphere relaxation is about $1.8 \mathrm{mM}^{-1} \mathrm{~s}^{-1}$ for all contrast agents. ${ }^{17-19}$ The contribution from second sphere relaxation is dependent on the ability of the ligand to closely associate via hydrogen bonding to water, but for discrete carboxylate-based chelates is typically around $0.8 \mathrm{mM}^{-1} \mathrm{~s}^{-1}$ (at $20 \mathrm{MHz}$ and $298 \mathrm{~K}$ ). ${ }^{19-21}$ The contribution from inner sphere relaxation in current contrast agents is about $2.0 \mathrm{mM}^{-1} \mathrm{~s}^{-1}$, but has the potential for optimization to much higher relaxivities. ${ }^{17}$

\subsection{Solomon Bloembergen Morgan Theory}

\subsubsection{Defining SBM Equations}

Solomon, Bloembergen, and Morgan (SBM) theory describes the inner and second sphere contributions. ${ }^{22-26}$ As inner sphere relaxation has the potential to be optimized in discrete chelates to provide highly effective agents, the parameters defined in SBM 
theory are the primary focus of this research. The equations of SBM theory (Equations $1.3-1.5)$ are routinely used for fitting relaxometric data to extract specific values for parameters effecting relaxivity. ${ }^{20,} 21,27,28$ Inner sphere relaxivity $\left(r_{1}{ }^{\mathrm{IS}}\right)$ is dependent upon the number of bound waters $(q)$, the rate of water exchange $\left(1 / \tau_{\mathrm{M}}\right)$, and the longitudinal inner-sphere water proton relaxation time constant $\left(\mathrm{T}_{1 \mathrm{M}}\right)$ as given by Equation 1.3.

$$
r_{1}^{I S}=\frac{q}{\left[H_{2} O\right]} \frac{1}{T_{1 M}+\tau_{M}}
$$

Equation 1.3: The inner sphere relaxation paramagnetic dependence The parameters that govern $\mathrm{T}_{1 \mathrm{M}}$, as shown by Equation 1.4, are the spin state $(S)$, the proton gyromagnetic ratio $\left(\gamma_{\mathrm{H}}\right)$, an electronic factor $(g)$, the Bohr magneton $(\beta)$, the proton and electronic Larmor frequencies $\left(\omega_{\mathrm{H}}\right.$ and $\left.\omega_{\mathrm{S}}\right)$, and the distance between the ion center and the proton $\left(r_{\mathrm{GdH}}\right)$.

$$
\frac{1}{T_{1 M}}=\frac{2}{15} \frac{{\gamma_{H}}^{2} g^{2} S(S+1) \beta^{2}}{r_{\mathrm{GdH}}{ }^{6}}\left(\frac{7 \tau_{c 2}}{1+\omega_{S}{ }^{2} \tau_{c 2}{ }^{2}}+\frac{3 \tau_{c 1}}{1+\omega_{H}{ }^{2} \tau_{c 1}{ }^{2}}\right)
$$

Equation 1.4: The longitudinal relaxation time constant of a bound water proton However, to simplify the discussion, $\mathrm{T}_{1 \mathrm{M}}$ is generally considered governed by the characteristic correlation time constant $\left(\tau_{\mathrm{C}}\right)$. The value of $\tau_{\mathrm{C}}$ is given by Equation 1.5 and is found to depend upon the electronic spin relaxation $\left(\mathrm{T}_{\mathrm{ie}}\right.$, where $i=1$ or 2 for longitudinal or transverse, respectively), the water residence lifetime $\left(\tau_{\mathrm{M}}\right)$, and the rotational correlation time $\left(\tau_{\mathrm{R}}\right)$. 


$$
\frac{1}{\tau_{c i}}=\frac{1}{T_{i e}}+\frac{1}{\tau_{M}}+\frac{1}{\tau_{R}}
$$

Equation1.5: Defining the correlation time constant associated with interactions at the Larmor frequency

\subsubsection{Narrowing down the SBM parameters}

There are many parameters in the SBM equations that are not controllable by ligand design, such as the Larmor frequencies (related to $\mathrm{B}_{0}$ field strength). There are other parameters for which control is not well understood, such as $\mathrm{T}_{1 \mathrm{e}}$ (influencing $\tau_{\mathrm{C}}$ based on interaction $\left(\Delta^{2}\right)$ and modulation $\left(\tau_{\mathrm{V}}\right)$ of zero-field splitting $)^{29}$ which has only been suggested to relate to chelate symmetry and rigidity. ${ }^{30-32}$ However, a few parameters in the above equations $\left(q, \tau_{\mathrm{M}}\right.$, and $\left.\tau_{\mathrm{R}}\right)$ have been identified as highly influential and easiest to control, therefore they have been well-researched in an effort to optimize contrast agent relaxivity.

The hydration number $(q)$ is most appropriately considered to be the number of vacant coordination sites available for occupation by water molecules. This parameter is readily controlled by altering ligand denticity. ${ }^{33}$ Increasing the value of $q$ increases relaxivity, but doing so requires a reduction in the denticity of the ligand, resulting in unacceptable levels of chelate stability. Therefore, the denticity of potential ligands for contrast agents is restricted to meet stability requirements, for this reason this work is focused on $q=1$ chelates. In nine-coordinate $(q=1) \mathrm{Gd}^{3+}$ chelates, water exchange occurs through a dissociative mechanism and is characterized by a large positive volume of activation $\left(\Delta \mathrm{V}^{\ddagger}\right) .{ }^{34}$ Due to the dissociative mechanism of exchange in these chelates, the hydration state is often considered to be a discrete value (typically $q=1$ or 0 ) as a water molecule is 
either coordinated or it is not. However, partial values of $q$ are commonly reported. Noninteger values of $q$ might suggest a time averaged water coordination value and reflect the time water spends on $v s$. off the chelate. Non-integer values of $q$ might also reflect a strong second sphere contribution; SBM theory does not differentiate one closely associated water molecule from two distantly associated water molecules. An example of this is the GdDOTP chelate that was thought to be $q=1$ for many years due to its high relaxivity values. ${ }^{35,}{ }^{36}$ However, the relaxivity of the $\mathrm{Gd}^{3+}$ chelate of $1,4,7,10$ tetraazacyclododecane-1, 4, 7, 10-tetrakis(methylenephosphonate) (DOTP) is in fact a result of its large second sphere contribution - caused by the increased hydrogen bonding to the phosphonate pendant arms of the complex (Figure 1.3). ${ }^{37,38}$

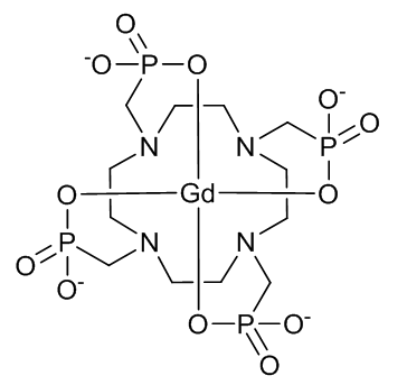

Figure 1.3: Connectivity of the GdDOTP chelate

Water residence lifetime $\left(\tau_{\mathrm{M}}\right)$ is the inverse of the water exchange rate constant $\left(1 / k_{\mathrm{ex}}\right)$. To optimize relaxivity it is important for the value of $\tau_{M}$ to have a balance such that protons need to reside on the metal ion long enough to be fully relaxed. For polyaminocarboxylate gadolinium chelates the optimum value of $\tau_{M}$ for $T_{1}$ contrast lies in the $10-40$ ns range and will depend on the $\mathrm{B}_{0}$ field. Clinically approved contrast agents currently have $\tau_{M}$ values on the order of $200-1000 \mathrm{~ns}$. Despite the slower than optimal 
water exchange rates these chelates remain in the fast exchange regime owing to the rapid rate of molecular tumbling which has a limiting effect on relaxivity. Water exchange rates can be controlled with various structural changes to the ligand which alter the environment around the interaction of the water with the metal. ${ }^{39,}{ }^{40}$ For instance, changing the ligand donor atoms of 1,4,7, 10-tetraazacycleododecane-1,4,7,10tetraacetate (DOTA) from acetate oxygens to atoms providing less electronic donation can have a drastic slowing effect on water exchange. ${ }^{39}$ Steric crowding of the water binding site has been shown to prevent the close approach of the water to the metal ion, resulting in an increase in water exchange rates. ${ }^{41}$ Altering the chelation ring size was studied in both macrocyclic and linear systems; it was found that substituting a propylene bridge for the ethylene bridge or a propionate group for an acetate donor can increase water exchange rates. ${ }^{42-44}$

Low molecular weight contrast agents tumble in solution at a much faster rate (on the order of $100-200 \mathrm{ps})$ than water exchanges. This causes the rotational correlation time ( $\left.\tau_{R}\right)$ to limit relaxivity. Slowing $\tau_{R}$ is possible with a variety of modifications to an agent. Two examples are increasing the size of the agent and binding it to a large, slowly moving (or static) substrate. ${ }^{38,45-48}$ As $\tau_{\mathrm{R}}$ slows, relaxivity increases and so does the influence of $\tau_{\mathrm{M}}{ }^{49}$ 


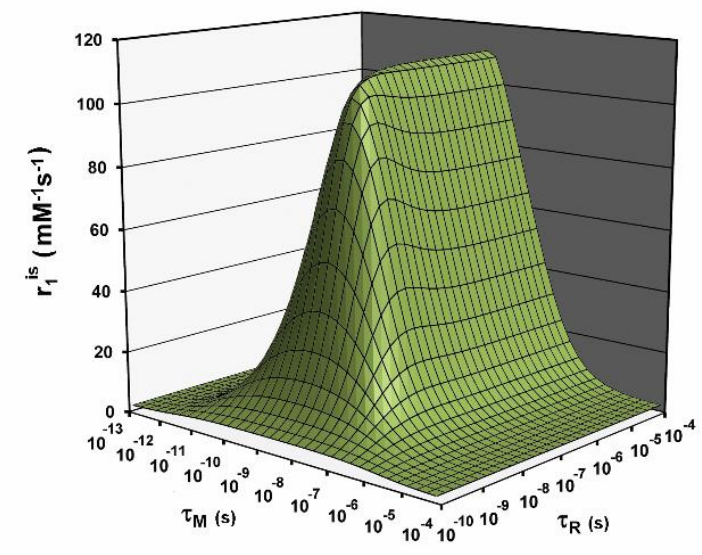

Figure 1.4: The SBM curve showing the dependence of inner sphere relaxation on $\tau_{\mathrm{R}}$ and $\tau_{\mathrm{M}}$ at $0.5 \mathrm{~T}$ imaging field.

By considering the effect of changing $\tau_{\mathrm{R}}$ and $\tau_{\mathrm{M}}$ on inner sphere relaxivity (Figure 1.4), it is it clear that slowing tumbling increases relaxivity and that there exists an optimal water exchange rate for maximum relaxivity. The optimal values of these parameters will vary with field. The relaxivity of current clinical contrast agents is limited by $\tau_{\mathrm{R}}$ values at are too fast. However, even when the rotational correlation times are sufficiently slowed for these agents, their $\tau_{\mathrm{M}}$ values are too slow to obtain the maximum relaxivity values predicted using SBM theory. ${ }^{40,50}$

\subsubsection{Relaxometric analyses of contrast agents}

The relaxivity of a contrast agent recorded across a range of external fields is known as a Nuclear Magnetic Resonance Dispersion (NMRD) profile. Fitting this profile to SBM theory allows certain parameters to be determined. This is possible through the field dependent parameters of the SBM equations: the correlation time constants impact relaxivity in proportion to the Larmor frequencies of the proton and the electron 
(Equations 1.4 and 1.5). A brief look at ${ }^{1} \mathrm{H}$ NMRD profiles can provide qualitative information about characteristics of the species being probed (Figure 1.5). A "hump" at high fields indicates a slowly tumbling species with long $\tau_{\mathrm{R}}$. Given that it is possible to speed water exchange beyond optimal kinetics, the effect of $\tau_{M}$ on the shape of the ${ }^{1} \mathrm{H}$ NMRD profile is not proportional. When comparing species with equal hydration the one with higher relaxivity across all fields is likely to have closer to optimal water exchange kinetics. Electronic relaxation also influences relaxivity, particularly at low fields, causing an offset of relaxation values with a greater effect at low fields.

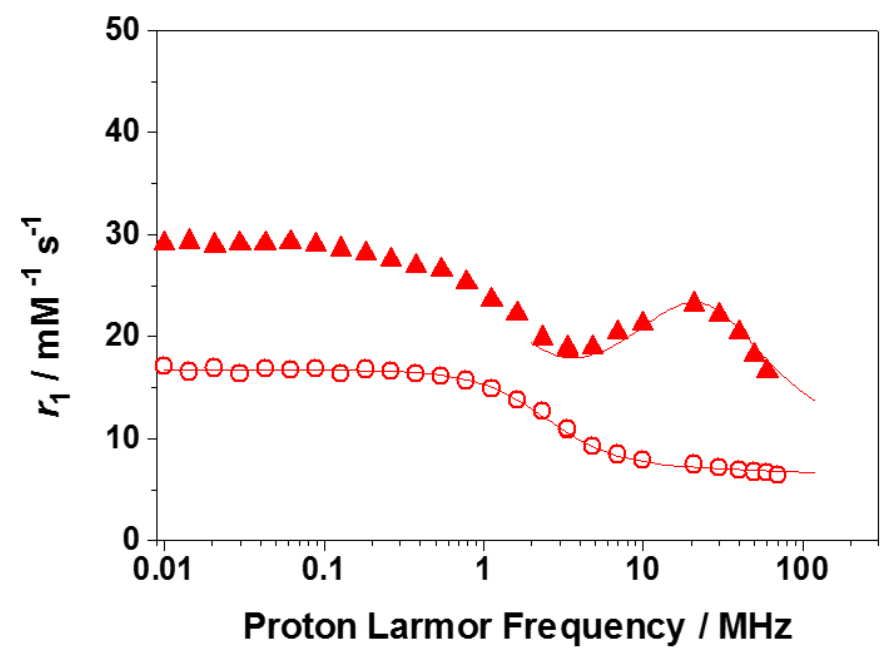

Figure 1.5: An example of a typical NMRD profile for chelates where $\tau_{R}$ is fast (open) and slow (solid). This profile, specifically, is for the corner regioisomer of GdBP-DOTA when the free and when bound to poly- $\beta$-cyclodextrin.

Qualitative analysis of these data can yield $\tau_{R}, \tau_{M}$, and $T_{1 e}$ values; the shortest of these values at a given field will both dominate and limit the relaxivity. The early literature includes discussion of how dramatic and unrealistic variations in parameter values can 
occur while still creating curves that appear to be good fits of the relaxometric data. For example, values of $r$ that are too long will result in a decrease in $q$ or a lengthening of $\tau_{\mathrm{R} .}{ }^{51}$ However, the fitting of these profiles has become routine as research in this field has progressed, and the discussion on possible ambiguity in the SBM fit has ceased. To minimize ambiguity in the SBM variables it is necessary to include empirical values for as many parameters as possible (for instance $\tau_{\mathrm{M}}$ is readily and independently measured by ${ }^{17} \mathrm{O}$ relaxometry). To minimize the number of variables during the fitting of the SBM equations some parameters are held constant. In current literature, $q$ and $r$ are often included in these "constants." 17,50 However, the parameter $q / r^{6}$ has been used previously as a single variable for hydration in fitting NMRD profiles..$^{20,52}$ We consider this to be a more accurate portrayal of hydration, as a result of the inherently joined relationship between $q$ and $r$.

\subsection{Contrast agent ligand design}

Contrast agents have been shown to be particularly effective at differentiating between tissues with similar relaxation characteristics, such as identifying cancerous regions and diseases of the central nervous system. ${ }^{53}$ Gadolinium is chosen for MR agents because it has the most unpaired electrons available on the periodic table, and it has the longest electronic spin relaxation of the lanthanides. ${ }^{54}$ Its anisotropic electronic shell and large electronic dipole are what make $\mathrm{Gd}^{3+}$ highly attractive as the paramagnetic metal in MR agents. However, the radius of gadolinium is similar to calcium and it will non-reversibly bind in calcium transport channels (such as those which enable the contraction of muscles - i.e., the heart). This makes $\mathrm{Gd}^{3+}$ toxic as a free ion in the body; therefore, ensuring the 
stability of these chelates is highly important. Although there are MRI contrast agents that are not based on $\mathrm{Gd}^{3+}$ (for example Feridex I.V. and Teslascan containing iron and manganese, respectively), all of the current clinically used MR agents contain $\mathrm{Gd}^{3+}$. The $\mathrm{Gd}^{3+}$ ion is chelated by octadentate polyaminocarboxylate ligands. These ligand structures are based on either (diethylenetriaminopentaacetic acid) DTPA or DOTA. GdDTPA (Magnevist) was the first to be approved for clinical use in 1988, followed by GdDOTA (Dotarem) in 1989. In the US however, GdDOTA is the most recent of the 7 agents to be approved for MRI of the central nervous system (March 2013). The most notable difference between these two gadolinium-based polyaminocarboxylate octadentate chelates is the linear backbone $v s$. the cage-like structure of the ligand (Figure 1.6). The cage-like structure of GdDOTA affords it a higher thermodynamic stability constant than that of GdDTPA: $\log \mathrm{K}_{\mathrm{ML}}=24.7$ and 22.3 , respectively. ${ }^{55}$ It is due to the higher stability of DOTA-type chelates that they are the common focus of research for extended functionality of contrast agents, such as potential targeting agents. With stability being the primary motivator, our research is focused on GdDOTA-type complexes.
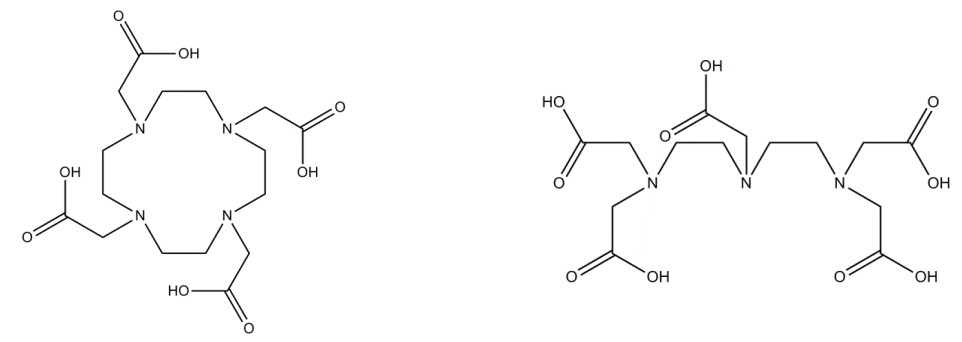

Figure 1.6: Ligands of clinically approved contrast agents (DOTA left; DTPA right). 
$\mathrm{H}_{4}$ DOTA was first published as a ligand for calcium in 1976, and was first considered as a chelating agent for rare earths by Desreux in $1980 .{ }^{56,57}$ LnDOTA has been noted in literature as being exceedingly stable across a broad $\mathrm{pH}$ range. ${ }^{56}$ The first crystal structure of a LnDOTA chelate was EuDOTA in $1984 .{ }^{56,58}$ It takes on the general structure of all LnDOTA-type chelates (Figure 1.7); when these complexes are viewed in a parallel plane the amino nitrogens of the macrocycle form the aza face of the chelate "below" the metal ion and the coordinating carboxylate oxygens form the negative face "above" the metal ion. The coordinated water in exchange with solvent is found in the axial position above the $\mathrm{O}_{4}$ plane. In the crystal, EuDOTA exists as a single species. However, in solution it has two species in exchange, as observed in the early work characterizing the geometry of LnDOTA-type systems. ${ }^{59}$

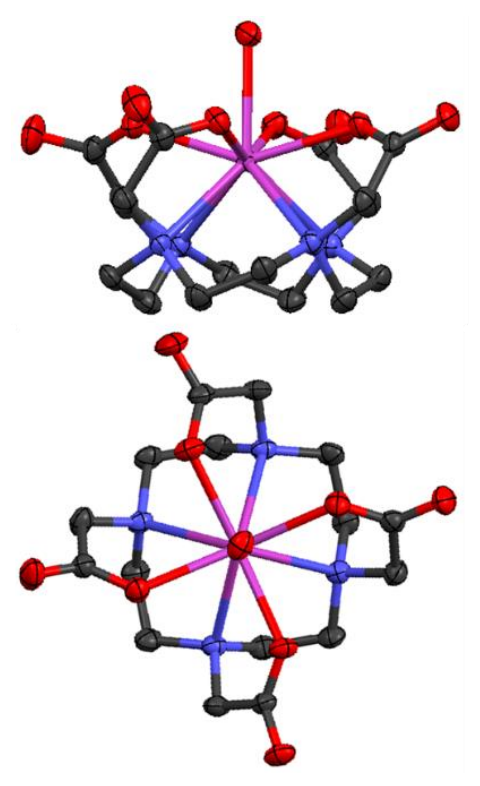

Figure 1.7: Side view (top) of EuDOTA crystal structure shows the relative positions of the coordinating nitrogens and oxygens and the apically located water. The view down the primary axis (bottom) shows square antiprism geometry. First published in $1984 .^{58}$ 


\subsection{Isomerism in LnDOTA chelates}

The chelation of LnDOTA results in five elements of helicity for the ethylene bridges and pendant arms: the pendant arms bind cooperatively ( $\Lambda$ or $\Delta)$ and independently of the orientation of each ethylene bridge ( $\lambda$ or $\delta$ ). In order to adapt for the size of lanthanide ions, the macrocycle always forms as a [3333] ring resulting in the same helicity for all four ethylene bridges. ${ }^{60}$ This affords LnDOTA chelates four conformations: two diastereoisomers and their enantiomeric pairs $(\Delta(\lambda \lambda \lambda \lambda)$ and $\Lambda(\delta \delta \delta \delta))$ and $(\Lambda(\lambda \lambda \lambda \lambda)$ and $\Delta(\delta \delta \delta \delta))$. When the chelate rings of the ethylene bridges share the same helicity as the pendant arms, it results in twisted square antiprism (TSAP) geometry; when they have opposite helicity, square antiprism (SAP) geometry results (Figure 1.8).

The structures of the diastereoisomers are distinguished by the twist angle of the $\mathrm{N}_{4}$ coordination plane and the $\mathrm{O}_{4}$ plane. A perfect square antiprism would have a $45^{\circ}$ torsion angle; the TSAP geometry describes a chelate whose two planes are rotated away from SAP geometry. The chelate torsion angles for hydrated LnDOTA-type chelates with coordinating carboxylate functional groups typically are found in the range of $35-41^{\circ}$ and $24-28^{\circ}$ for SAP and TSAP chelates, respectively. In order to adapt to these coordination geometries, the pendant arms take different positions relative to the $\mathrm{N}_{4}$ and $\mathrm{O}_{4}$ planes. In TSAP geometry the arms rest almost perpendicular to the $\mathrm{N}_{4}$ and $\mathrm{O}_{4}$ planes, which results in a taller coordination cage in TSAP geometry than seen in the SAP geometry. 

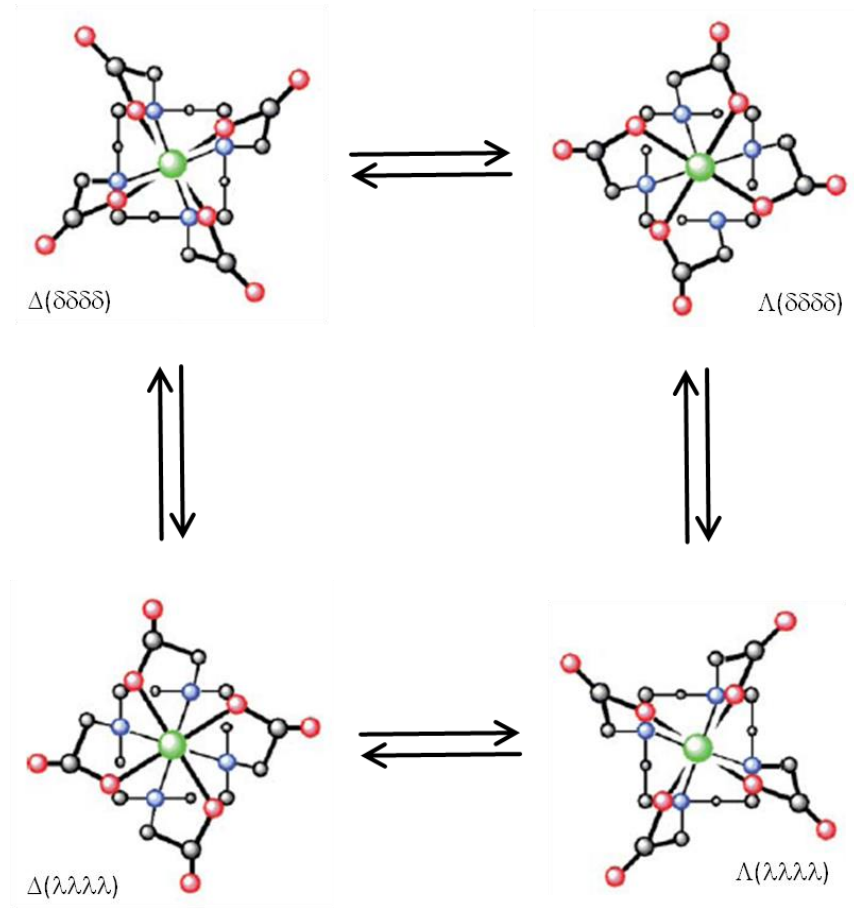

Figure 1.8: Interconversion of diastereoisomers in DOTA-type chelates via pendant arm rotation and ethylene bridge inversion.

With no steric hindrance on the macrocyclic ring or at the $\alpha$-position of the pendant arm, the helicity of these elements can invert through either a flip of the macrocyclic ring or rotation of the pendant arms. LnDOTA in solution readily converts between conformations (Figure 1.8). The interconversion between diastereoisomers is slow on the NMR timescale: ${ }^{1} \mathrm{H}$ and ${ }^{13} \mathrm{C}$ NMR spectra show a set of peaks for both the SAP and TSAP conformations. ${ }^{61}$ This allows the diastereoisomers in solution to be distinguished by their NMR spectra (Figure 1.9). 


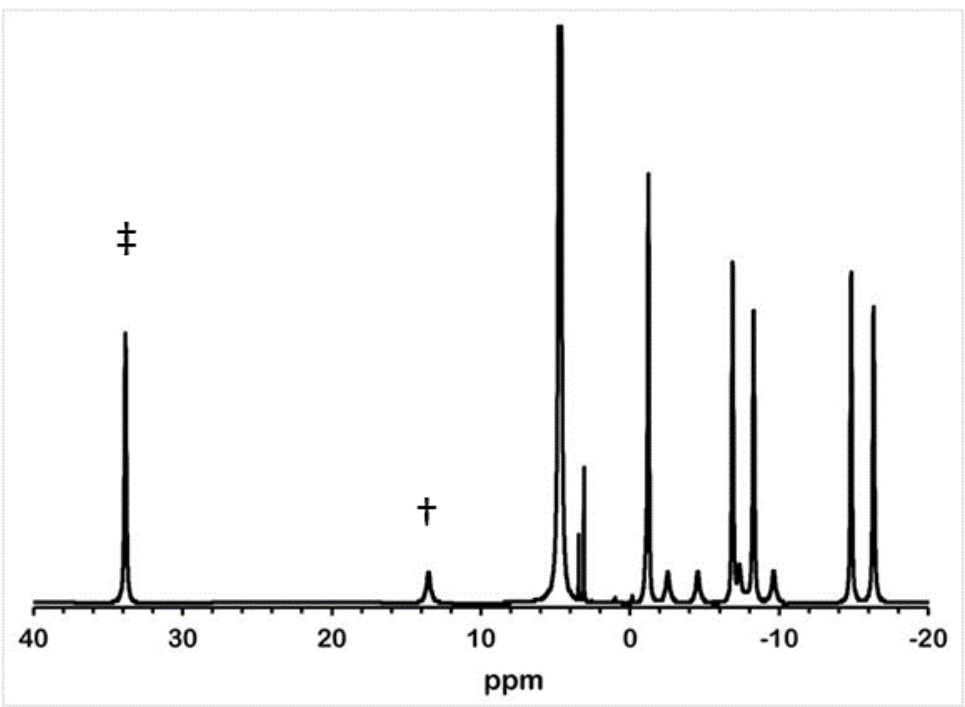

Figure 1.9: ${ }^{1} \mathrm{H}$ NMR spectrum of EuDOTA showing two geometric isomers in solution; these are readily identified by the most shifted axial proton (in the range of 10-20 ppm for TSAP geometry $(\dagger)$ and 30-40 ppm for SAP geometry $(\ddagger))$.

The TSAP coordination isomer exchanges water 50 - 100 times faster than the SAP isomer. This difference in water kinetics is a result of differences in the coordination cage provided by the ligand; the cage of the TSAP isomer is much taller than that of the SAP isomer. The taller coordination cage of the TSAP isomer is a result of the more perpendicular orientation of the pendant arms to the $\mathrm{O}_{4}$ and $\mathrm{N}_{4}$ planes. This causes an increased electrostatic repulsion between the carboxylate donors of the $\mathrm{O}_{4}$ plane and the oxygen of the water molecule, and prevents the close approach of water in the TSAP isomer. In solution the TSAP and SAP species of LnDOTA chelates are in exchange. The difference in size of the coordination cage of the two geometric isomers causes the major isomer in solution to be dependent on the size of the $\mathrm{Ln}^{3+}$ ion (Figure 1.10). ${ }^{46}$ 


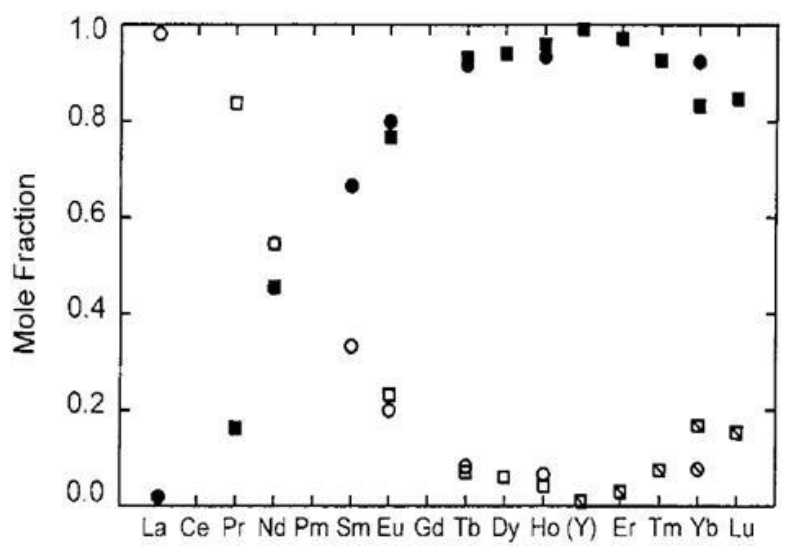

Figure 1.10: Geometric isomer distribution in solution of LnDOTA complexes. TSAP isomers (open) and SAP isomers (closed).

As seen in Figure 1.10, LnDOTA chelates at the beginning of the series display predominantly TSAP geometry. With ionic contraction across the series the SAP geometry becomes more prevalent starting at $\mathrm{Nd}^{3+}$ and reaching $95 \%$ in solution with $\mathrm{Gd}^{3+}$ chelates. This trend begins to reverse at $\mathrm{Ho}^{3+}$ : the TSAP population increases, but stays the minor species in solution. ${ }^{62,63}$ The SAP geometry is hydrated for all $\mathrm{Ln}^{3+}$ ions. Unlike the SAP geometry the TSAP geometry changes coordination number across the lanthanide series with ionic contraction. Early in the series the LnDOTA chelates in TSAP geometry are primarily hydrated $(\mathrm{CN}=9)$, but later in the series these chelates become increasingly dehydrated $(\mathrm{CN}=8)$. The dehydrated TSAP geometry is often denoted as TSAP' in the literature. ${ }^{62}$ However, considering TSAP' (the dehydrated TSAP isomer) as a separate species is inaccurate. Were there to be a TSAP' species in solution that is not in fast exchange with the TSAP species it would be visible in the ${ }^{1} \mathrm{H}$ NMR spectrum. 


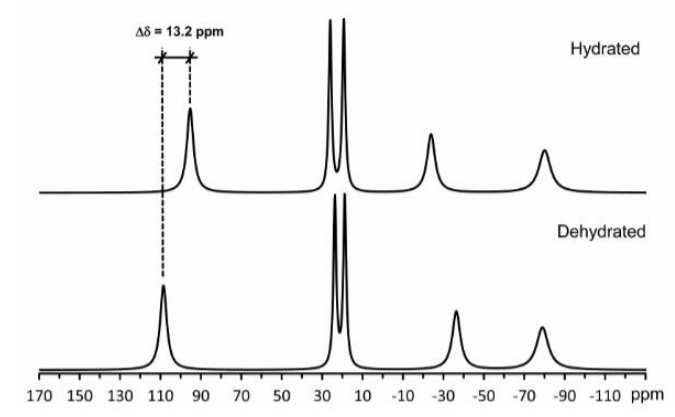

Figure 1.11: ${ }^{1} \mathrm{H}$ NMR simulated spectra of $\mathrm{YbDO} A \mathrm{AP}(\mathrm{ABn})$ based on the proton distances found in the hydrated and dehydrated chelates of the YDOTA-type crystal structure. ${ }^{64}$

${ }^{1} \mathrm{H}$ NMR spectra for a DOTA-type $\mathrm{Yb}^{3+}$ chelate protons can be simulated for both hydrated and dehydrated species from the different chelate structures found in the crystal structure of YDO3A(ABn) from Lukeš (Figure 1.11). On the basis of the ligand field parameter used in this simulation, the axial side protons would have a $13.2 \mathrm{ppm}$ difference in chemical shift between the hydrated and dehydrated chelates. If there were a TSAP' species either in slow exchange or not exchanging with the TSAP species, it would be readily apparent in ${ }^{1} \mathrm{H}$ NMR spectra by the presence of a second set of proton peaks. YbDOTA would be an ideal system in which to see if the hydrated SAP isomer would be in exchange with the hydrated TSAP species, but most of the TSAP species is expected to be dehydrated due to the size of the $\mathrm{Yb}^{3+}$ ion. Thus a sizable population of TSAP' should be visible in the NMR spectrum. However, the ${ }^{1} \mathrm{H}$ NMR spectrum of YbDOTA shows only a single set of peaks for TSAP geometry and a single set of peaks for the SAP geometry. This suggests that the idea of a separate species of TSAP' being in solution is incorrect. 
Clearly, some of the chelate molecules are dehydrated in solution, but they represent the intermediate of an exchange process. The prevailing theory in the field divides water in the system as either closely or distantly associated with the chelate. That the two species ("hydrated" and "dehydrated") are always in exchange suggests that a new theory, which can apply to water on a continuum, is necessary. This is not a new idea: Parker and coworkers previously identified that the distinction between hydration spheres is strictly arbitrary and that there is a need for one theory which incorporates all water contributions seamlessly ${ }^{65}$ When the water is very far away (greater than $4 \AA$ ), the relaxation theory defined by diffusion works well. And when water is very close and in exchange, the SBM equations also apply well. It is when the water is located at the edges of these spheres that the theoretical models start to break down. Accurately measuring the value of $r$ in solution is difficult and an approximation is often described by the solid state values for fitting solution data.

\subsection{Preventing helicity inversion in LnDOTA-type chelates}

In LnDOTA chelates the two geometric isomers (SAP and TSAP) readily exchange by inversion of the ethylene bridges and rotation of the pendant arms. ${ }^{59,61,66}$ The exchange between the geometric isomers results in large conformational changes in the chelate. These obscure any minor structural differences that may occur with water exchange in these chelates in solution. It is possible with steric hindrance to prevent inversion of helicity of both the pendant arms and the macrocycle.

Substitution at the $\alpha$-carbon of the pendant arm will lock-out the arm rotation, but still allow for the inversion of the ethylene bridges of the macrocyclic ring. Similarly, bulky 
groups substituted on the macrocyclic ring will lock-out bridge inversion, but still allow for rotation of the pendant arms. When both of these types of ligand substitutions are employed in ligand design we are able to lock-in a specific coordination geometry in the chelate. ${ }^{67-70}$ Preference for a specific coordination geometry can be established with as little as one methyl substitution on one acetate pendant arm or one ethylene bridge of the macrocycle. Additional (as well as larger) substitutions to the other pendant arms or ethylene bridges increase the preference for a specific coordination geometry. ${ }^{71-75}$ For example, some have used methyl substitutions on each ethylene bridge of the macrocycle to prevent chirality inversion, but we employ a single nitrobenzyl substituent to lock-out inversion of helicity of the macrocyclic ring. ${ }^{70,71,76}$

The nitrobenzyl substituent is used as a stable moiety for study that can be readily modified to interact with macromolecules. The addition of the benzylic moiety to the macrocycle introduces a third element of helicity that is created with the chelation of $\mathrm{Ln}^{3+}$ ions. The bulky nature of the benzylic substituent requires a pseudo-equatorial position in relation to the chelate. The equatorial protons of the corner and side carbons on the macrocycle are pro- $S$ - the benzylic group can be located in the equatorial position on either carbon. ${ }^{68}$ With inversion of the [3333] macrocyclic ring prevented, the corner and side carbons are nonequivalent (Figure 1.12). This gives rise to two sets of structural isomers: the corner and side regioisomers. 
A

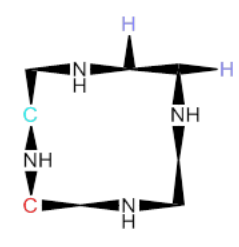

B

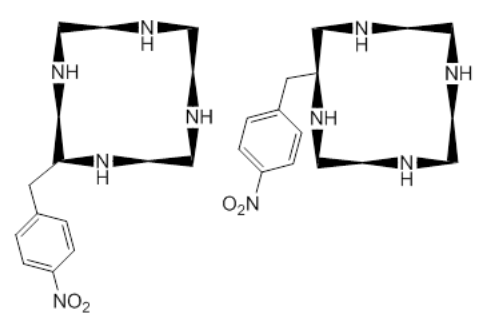

Figure 1.12: On the [3333] macrocyclic ring orientation the $(A)$ pro- $S$ protons (purple) and the locations of corner (red) and side (blue) carbons and (B) position of the nitrobenzyl substituent on corner or side carbons.

$\mathrm{Ln}^{3+}$ chelates of ligands that have methyl substitutions on the pendant arms or addition of the benzylic substituent to the macrocycle maintain comparable values to $\mathrm{Ln}^{3+}$ chelates of the parent DOTA ligand (Table 1.1).

Table 1.1: Thermodynamic stability constants for $\mathrm{Gd}^{3+}$ chelates of DOTA, ${ }^{55}$ DOTMA, ${ }^{63,77}$ and NB-DOTA. ${ }^{72}$

\begin{tabular}{cccc}
\hline & DOTA & DOTMA & NB-DOTA \\
\hline$K_{\mathrm{GdL}}$ & 24.7 & 23.6 & 24.2 \\
\hline
\end{tabular}

\subsection{Hydration of the geometric isomers and its effect on relaxivity}

The lack of geometric isomer exchange in our rigid chelate systems allows us to control the stereochemistry of the ligand in order to isolate specific chelate coordination chemistry. This enables the novel and detailed investigation into parameters of water exchange in these systems. From these rigid chelates it has been determined that chelates 
of TSAP geometry, with its very fast water exchange kinetics, have a lower hydration state $\left(q / r^{6}\right)$ than comparable chelates in SAP geometry. Study of both solid and solution states supports this deduction. The crystals of TSAP and SAP geometries have clearly different water-lanthanide distances: the $r_{\mathrm{GdO}}$ distances in the crystal structures of $\mathrm{Gd}^{3+}$ chelates of 1, 4, 7, 10-tetrakis(carboxyethyl)-1, 4, 7, 10-tetraazacyclododecane (TCEDOTA) and 1, 4, 7, 10-tetraazacycleododecane-1, 4, 7, 10-( $\alpha$-methyl)tetraacetate (DOTMA) are 2.343 and $2.500 \AA$, respectively. ${ }^{57,65}$ This is a $2.76 \%$ difference between the SAP and TSAP isomers. Differences in hydration of TSAP and SAP isomers in the solution state can be seen in the luminescent quenching experiments of the $\mathrm{Eu}^{3+}$ chelates of (S)-2-(4-nitrobenzyl)-1, 4, 7, 10-tetraazacycleododecane-1, 4, 7, 10-( $\alpha$ methyl)tetraacetate (NB-DOTMA) chelates which resulted in $q=0.74$ and 0.97 for the TSAP and SAP isomers, respectively, using a modification to Horrocks' method. ${ }^{64,69}$ This is a $31.1 \%$ difference in hydration of the TSAP and SAP geometries (for comparison to the solid state values, this is equivalent to a $4.5 \%$ increase in $r_{\mathrm{LnH}}$ ). This empirically determines that the hydration state for the EuNB-DOTMA TSAP isomer is lower than the SAP isomer. As a result of the complexity of the SBM equations, we represent this difference in hydration states by utilizing different $r_{\mathrm{GdH}}$ values in the process of fitting ${ }^{1} \mathrm{H}$ NMRD profiles. 

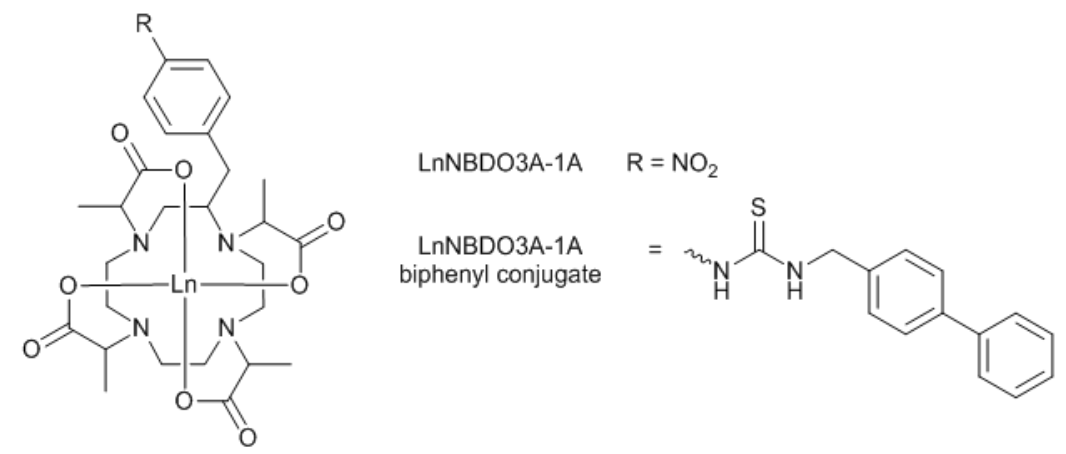

Figure 1.13: Connectivity of LnNB-DO3A-1A and its biphenyl conjugates

The loss of hydration with very fast water exchange has an impact on the relaxivity of these contrast agents in systems where $\tau_{R}$ can be slowed sufficiently for $\tau_{M}$ to limit relaxivity. For instance in the biphenyl conjugates of GdNB-DO3A-1A (Figure 1.13), despite having a $\tau_{M}$ value closer to the predicted optimal value (Table 1.2), the TSAP isomer of the biphenyl conjugate of GdNB-DO3A-1A has lower relaxivity than the SAP isomer. This identifies a discrepancy between the optimal parameters predicted from theory and empirical practice.

Table 1.2: Water residence lifetime $\left(\tau_{M}\right)$ and relaxivities $\left(r_{1}\right)$ for the corner isomers of GdNB-DO3A-1A biphenyl conjugates when bound to poly- $\beta$-cyclodextrin at $298 \mathrm{~K} .{ }^{28}$

\begin{tabular}{ccc}
\hline & SAP & TSAP \\
\hline$\tau_{\mathrm{M}}(\mathrm{ns})$ & 70 & 8 \\
$r_{1}$ at $20 \mathrm{MHz}\left(\mathrm{mM}^{-1} \mathrm{~s}^{-1}\right)$ & 46 & 30 \\
\hline
\end{tabular}


We have suggested that this unexpected result is a product of reduced hydration state $\left(q / r^{6}\right)$ in chelates with very fast water exchange. ${ }^{28}$ The very fast water exchange of these TSAP chelates increases the amount of time the water spends off the chelate - effectively decreasing hydration in these chelates by lengthening the average distance of the water from the metal in the distribution of chelates in solution at any given moment. With the relaxivity dependence of $r^{-6}$ a difference in as little as $0.2 \AA$ would result in a $50 \%$ increase in relaxivity. ${ }^{17}$ This indicates that the optimal $\tau_{M}$ value in practice in will be slower than predicted by theory.

\subsection{Using other $\mathrm{Ln}^{3+}$ ions for probing GdDOTA-type systems}

It is the same characteristics of gadolinium that make it ideal for $\mathrm{T}_{1}$ and $\mathrm{T}_{2}$ relaxation that also make it poor for the determination of structural characteristics by NMR. Fortunately for research involving lanthanides, despite the ionic contraction across the series, the lanthanides all have similar coordination chemistry and neighboring ions are of similar size. With the distinct physiochemical properties of the various lanthanides, it is advantageous to be able to change the metal ion center, as this opens many options for probing the properties of these chelates. The coordination similarities resulting from the buried nature and delocalization of the f-orbital electrons allow for the ligand characteristics of one complex to be reliably applied to the complex of the neighboring lanthanide. For this reason, it is common for researchers in this field to make more than one $\mathrm{Ln}^{3+}$ complex of a ligand in order to probe the other relevant properties of the complex. For example, $\mathrm{Eu}^{3+}$ and $\mathrm{Tb}^{3+}$ have visible luminescence with long (ms) lifetimes. ${ }^{78}$ Hydration of their chelates can be determined by a modified Horrocks' 
method, ${ }^{58,67}$ and these values are frequently attributed to the comparable $\mathrm{Gd}^{3+}$ chelates, as $\mathrm{Eu}^{3+}$ and $\mathrm{Tb}^{3+}$ are neighboring ions to $\mathrm{Gd}^{3+}$ with similar sizes. ${ }^{64,69}$ In addition, many of the $\mathrm{Ln}^{3+}$ ions provide large paramagnetic ${ }^{1} \mathrm{H}$ proton shifts ${ }^{79}$ that aid in the identification of structural relationships within the ligand; the structures of $\mathrm{Gd}^{3+}$ chelates are often assumed to be comparable to those of the $\mathrm{Eu}^{3+}$ chelates.

Despite the common analogy drawn in literature of billiard balls when referencing the lanthanide series, there are slight differences expected between their chelates. As the lanthanide ions contract across the series, their coordination preferences switch from nona- to octadentate. This has impact both on their coordination geometries and their water exchange rates. It is projected that the middle lanthanide ions will have the fastest water exchange rates because of their more flexible coordination preferences. When both nine and eight coordination spheres are acceptable, the energy barrier to exchange is at its smallest and exchange is fast. This suggests that for $\mathrm{Gd}^{3+}$ ions, dissociation is the major contributor to the interchange mechanism of water exchange. ${ }^{80}$ The ionic contraction across the series is also expected to slightly shorten bond distances as the effective nuclear pull increases, but the relative positions of the donor atoms should be consistent across the series.

\subsection{This Work}

Current clinical contrast agents primarily image water in blood; the future of MRI contrast envisages agents with sufficient relaxivities to enable imaging macromolecules associated with specific pathologies. Clinical MRI employs comparatively high fields starting at $1.5 \mathrm{~T}(64 \mathrm{MHz})$, at these high fields $\tau_{\mathrm{R}}$ is limiting for the low molecular weight 
agents such as those of currently clinically approved agents. This means that the relaxivity of clinical contrast agents is quite low. There are many relatively facile methods for lifting this limiting parameter. ${ }^{38,45-48}$ Study of these slowly tumbling BFCs have indicated that there is still much to be gained in the understanding of how some SBM parameters are influenced by water exchange. In order to achieve sufficiently improved relaxivity desired for BFCs, there is a need for improved understanding of the full influence of water exchange.

There is currently a discrepancy between theory and empirical practice when predicting the values for parameters that will yield the most efficient contrast agent. Our group has proposed that this stems from a lack of prioritizing the importance of water proton distance $\left(r_{\mathrm{LnH}}\right)$ as a representation of hydration $\left(q / r^{6}\right)$ in the process of fitting the SBM equations. Overlooking $r_{\mathrm{LnH}}$ as a dynamic parameter is a result of the complexity of the SBM equations. It is necessary to hold some of these variables constant in order to fit relaxometric data. Despite having some precedence in the literature, using $q / r^{6}$ as a single parameter in the SBM equations is in direct contrast to the current interpretation of SBM theory. ${ }^{54,81}$ Common practice includes the parameters $q$ and $r$ as "constants" with the rationale that the hydration of the system is constant. Indeed, an optimal water exchange rate of about $10 \mathrm{~ns}$ can be calculated using $q=1$ and $r_{\mathrm{GdH}}=3.1 \AA .{ }^{17}$ However, hydration of the chelate is dynamic and we have proposed that it decreases with very fast water exchange.

There has been relatively little examination into the situation where water-lanthanide distance becomes intermediate to the $q=1$ (fully hydrated) chelate and the $q=0$ (fully 
dehydrated) chelate. To investigate the relationship of structure and water exchange, we will explore $\mathrm{Ln}^{3+}$ complexes with $\mathrm{H}_{4}$ DOTMA, $\mathrm{H}_{4} \mathrm{NB}$-DOTA and $\mathrm{H}_{4} \mathrm{NB}$-DOTMA. LnDOTMA complexes have a methyl substituent at each of the $\alpha$-carbons of all four pendant arms. LnNB-DOTA and LnNB-DOTMA complexes have the same benzylic substituent: the LnNB-DOTA has the benzylic substituent on one ethylene bridge, but no substitutions to the pendant arms; the LnNB-DOTMA has a benzylic substituent on one ethylene bridge and methyl groups at each of the $\alpha$-carbons of all four pendant arms. 
CHAPTER 2: STRUCTURAL VARIATIONS IN THE CRYSTAL STRUCTURES OF DOTMA CHELATES ACROSS THE LANTHANIDE SERIES MAY REFLECT CONFORMATIONAL CHANGES ASSOCIATED WITH WATER EXCHANGE IN SOLUTION

\subsection{Crystal structures: a window into solution state behavior}

Crystal structures do not necessarily reflect the structures of molecules in solution. Furthermore, the static nature of a single crystal structure is unable to reflect the dynamic nature of the solution state. It is critical that analysis of crystal structure data be judiciously considered if it is to be applied to developing an understanding of solution state chemistry. Solid state data allows for analysis of the molecular structure without obfuscation of motions associated with solution. In 2006 Lukeš and Hermann published the YDO3A-P(ABn) crystal structure (Figure 2.1). This structure is intriguing because it contains - in a single unit cell - the same chelate in different conformations. Of particular interest were two chelate structures that adopted the same coordination geometry (TSAP) but have different values of metal hydration (Figure 2.2). ${ }^{82}$

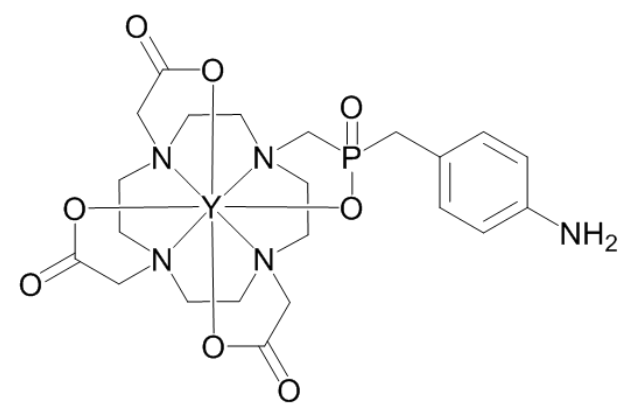

Figure 2.1: Connectivity structure of YDO3A-P(ABn) 


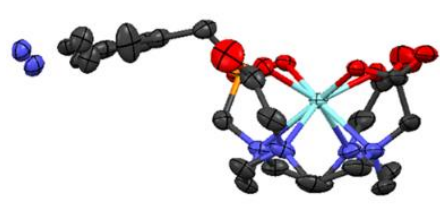

Dehydrated TSAP

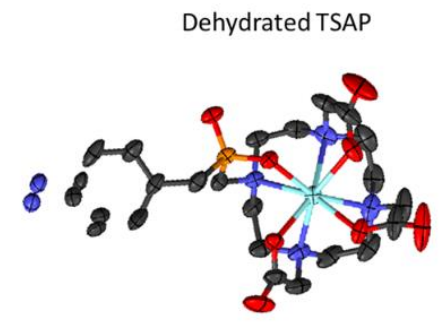

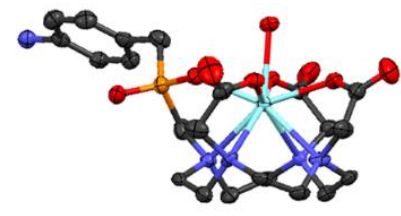

Hydrated TSAP

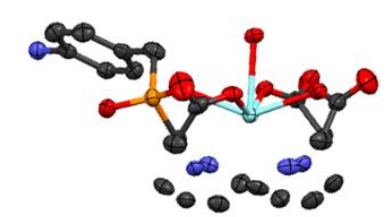

Hydrated SAP

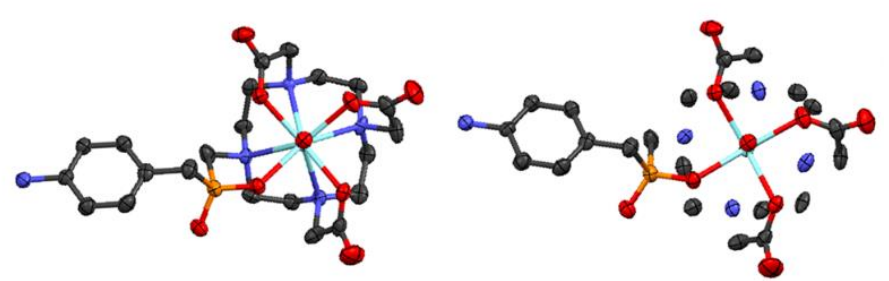

Figure 2.2: Structures of the YDO3A-P(ABn) complexes in the crystal showing three chelates: TSAP', TSAP, and SAP (left, middle, and right, respectively).

It is apparent at first glance that these three complexes vary in the number and position of donor atoms in the coordination cage. Closer inspection of the chelate parameters reveals structural differences between the hydrated and dehydrated species of the same geometry (the TSAP and TSAP' chelates, respectively). Of particular note is the location of the metal in the coordination cage. Hermann and Lukeš described this using an angle between the metal and two coordinating oxygens; Sherry, et al. have used the $\mathrm{d} / \mathrm{c}$ ratio to indicate the relative position of the metal in the coordination cage. ${ }^{19}$ The $\mathrm{d} / \mathrm{c}$ ratio indicates the location of the metal relative to the $\mathrm{N}_{4}$ and $\mathrm{O}_{4}$ planes (Figure 2.3): the distance from the $\mathrm{N}_{4}$ plane to the lanthanide ion (d) and the $\mathrm{N}_{4}$ plane to the $\mathrm{O}_{4}$ plane (c). We find this a more direct method for describing the location of the metal than the angle between the metal and the coordinating oxygens, as that angle is a result of both the drop in the cage and the decrease in size of the $\mathrm{O}_{4}$ area. 


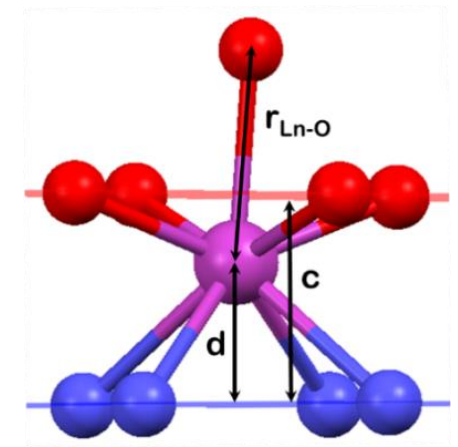

Figure 2.3: The coordination cage of LnDOTA-type chelates. Crystal structure parameters of the complex relevant to hydration values

It is in the Lukeš and Hermann structures that the relationship between the position of the metal in the coordination cage and the presence of an apical water molecule was first observed. Structural assessment of these complexes indicates that not only does the metal move up and down in the chelate during water exchange, but that there are other conformational changes in the chelate associated with the motion of the metal (Table 2.1). These observations ignited the idea that water exchange in LnDOTA chelates causes a "breathing" motion in the ligand. 
Table 2.1: Select parameters for the three $\mathrm{Y}^{3+}$ chelates in the Lukeš and Hermann structure (seen in Figure 2.2).

\begin{tabular}{cccc}
\hline Chelate & SAP & TSAP & TSAP' \\
\hline rYo $[\mathrm{Y}-\mathrm{O}(\mathrm{W})]$ & 2.485 & 2.485 & N/A \\
Y-Q $(\mathrm{O})$ & 0.761 & 0.761 & 1.028 \\
$\mathrm{Y}-\mathrm{Q}(\mathrm{N})$ & 1.582 & 1.660 & 1.531 \\
$\mathrm{~d} / \mathrm{c}$ & 0.675 & 0.686 & 0.598 \\
\hline
\end{tabular}

The "breathing" motion is a result of the metal ion moving within the coordination cage and can be understood in terms of the Lewis acidic nature of the $\mathrm{Y}^{3+}$ ion; after the loss of electronic density resulting from water dissociation, the ion seeks to regain electronic density by moving closer to the $\mathrm{N}_{4}$ plane. As the metal ion drops toward the $\mathrm{N}_{4}$ plane, the carboxylate oxygens pull together above the metal ion - this motion of the pendant arms resembles "breathing" in the chelate. Distances between the metal and the centroids of the $\mathrm{N}_{4}$ and $\mathrm{O}_{4}$ planes (referred to as $\mathrm{Q}(\mathrm{N})$ and $\mathrm{Q}(\mathrm{O})$, respectively) are used to determine the relative position of the metal in the coordination cage.

Conformational changes associated with water exchange have the potential to influence how we approach the parameter of hydration of contrast agents. In particular, these motions suggest the distance between the $\mathrm{Ln}^{3+}$ metal ion and the apical associated water 
molecule $\left(r_{\mathrm{LnO}}\right)$ may not be constant for all chelates: this parameter proportionally influences the relaxivity by $r^{-6}$.

In most DOTA-type chelates with rare earth metals, such as those commonly studied in the development of new MRI contrast agents, these comparatively small "breathing" motions are obscured by the larger conformational motions associated with exchange between geometric isomers. Our lab is able to synthesize chelates with no isomer exchange. This allowed us to recently investigate these conformational "breathing" motions in LnDOTA-type systems without obfuscation of geometric isomer exchange, and compare it with a DOTP chelate that has no inner sphere water. ${ }^{64}$ For this analysis variable temperature ${ }^{1} \mathrm{H}$ NMR spectra were collected and the dipolar shifts of the proton resonances for our isolated geometric isomers and a DOTP complex were analyzed to confirm the presence of this "breathing" motion. The resonances for protons on the chelate without water exchange (DOTP) had different shift patterns than the chelates with water exchange. This analysis indicates that there are structural changes in the chelates associated with the increase of water exchange as result of increased temperature.

\subsection{A survey of crystallographic data of LnDOTA-type chelates}

Further support for the idea that LnDOTA-type chelates undergo a "breathing" motion in solution can be found in solid state structures. Published crystallographic data of LnDOTA-type chelates were assessed in terms of the position of the metal ion within the coordination cage as a function of hydration number. ${ }^{21}$, 27, 37, 39, 40, 73, 83-89 Only LnDOTA-type ligands incorporating acetate, amide, alcohol, phosphinate, phosphonates, and pyridyl donor atoms on the pendant arms were included (Figure 2.4). 

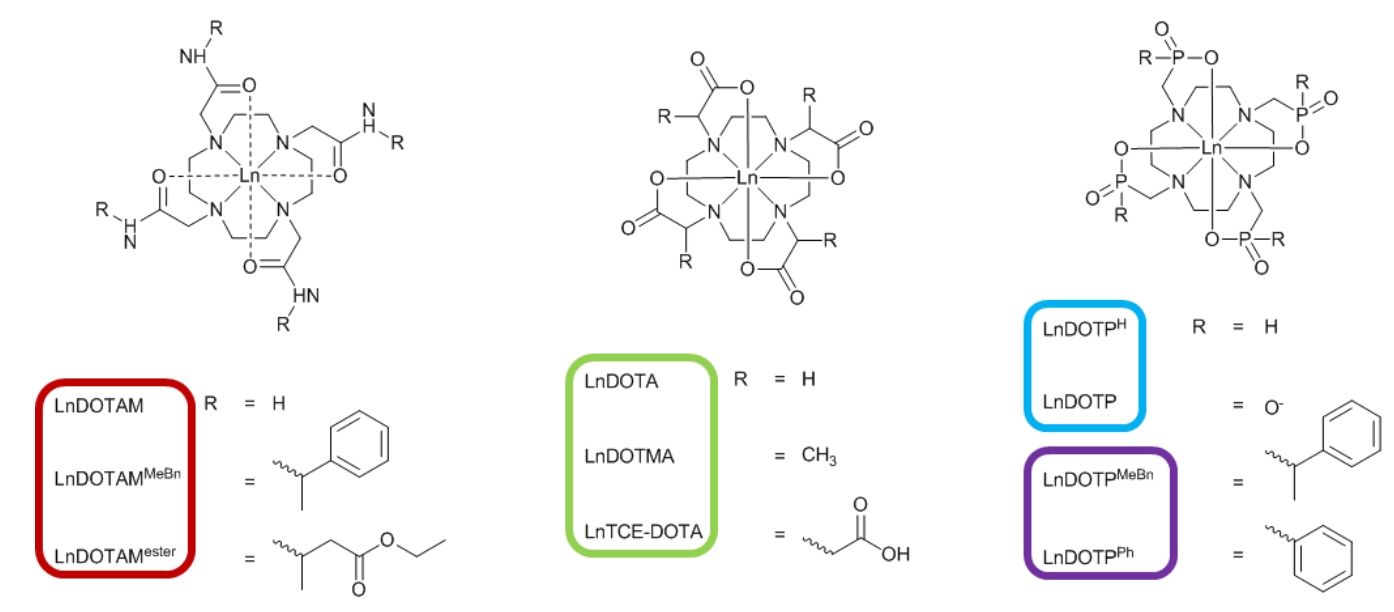

Figure 2.4: Connectivity structures of LnDOTA-like chelates associated with the crystal structures of $\mathrm{Ln}^{3+}$ chelates in literature survey.

Colored boxes are coordinated with Figure 2.5.

The LnDOTA-type literature survey of crystal structures revealed different structural trends in SAP and TSAP chelates (Figure 2.5) - the size of metal ion and the geometric isomer reflect the hydration of the complex. The larger lanthanides prefer a coordination number $(\mathrm{CN})$ of 9 ; the smaller lanthanides prefer a $\mathrm{CN}$ of 8 . Across the series chelates with SAP geometry are $\mathrm{CN}=9$, while the LnDOTA-type chelates in SAP geometry in the crystal are all hydrated $(q=1)$ for the entire lanthanide series. The TSAP geometry affords a larger coordination cage, so in the early series it is $\mathrm{CN}=9$ for the larger lanthanides and in the late series it is $\mathrm{CN}=8$ for the smaller lanthanides. This means that complexes in TSAP geometry are hydrated earlier in the lanthanide series and become dehydrated later in the series. This dehydration is a result of the ionic contraction which causes steric crowding of the apical binding site. 


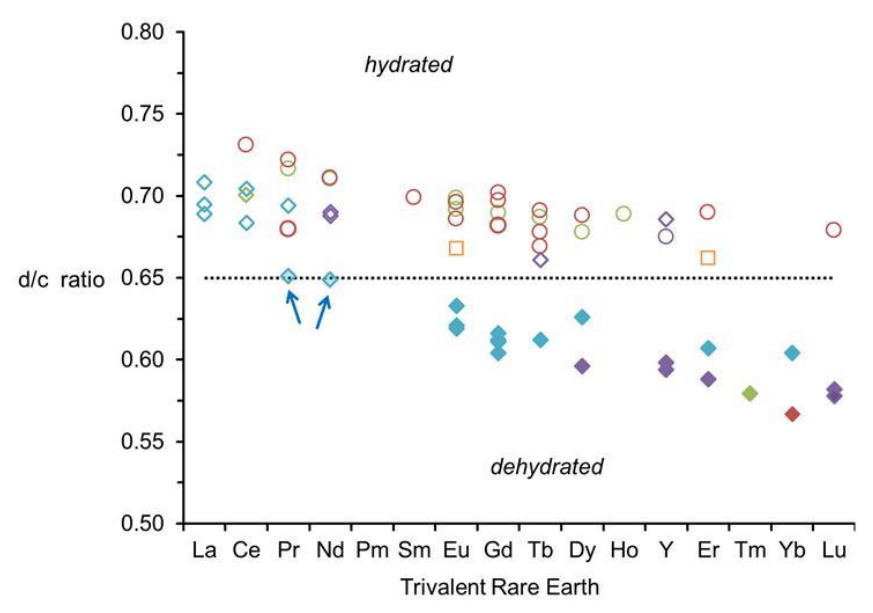

Figure 2.5: $21,27,37,39,40,73,83-89$ Compiled d/c ratios from LnDOTA-type chelates.

Diamonds $=$ TSAP, squares $=$ SAP. Open and closed data points are $q=1$ and 0 , respectively. Green, red, orange, blue, and purple represent complexes with pendant arms containing acetates, amides, pyridyls, phosphinates and phosphonates, respectively. Arrows indicate the few structures that provide insight for partially hydrated chelates. Linear correlation for TSAP data $y=-0.0094 x+0.7002\left(R^{2}=0.9071\right)$, for SAP data $y=-0.004 x+0.7244\left(R^{2}=0.6808\right)$.

The position of the ion in the ligand cage for LnDOTA-type chelates is a function of the effective nuclear charge/size of the lanthanide ion. The relative positioning of the metal in the coordination cage (represented by the $\mathrm{d} / \mathrm{c}$ ratio) seems to indicate hydration. At some point the metal ion drops sufficiently far into the coordination cage, causing the contraction of the donor atoms above the metal ion, and providing sufficient steric crowding to push the apical water molecule out of the coordination cage. We chose to analyze the literature structures by $\mathrm{d} / \mathrm{c}$ ratios as a result of the conformational changes seen in the Lukeš and Hermann crystal (Figure 2.2). The d/c ratio appears to be a potential indicator of the hydration in these chelates - the ability for the $\mathrm{d} / \mathrm{c}$ ratio to 
accept a range of values allows the discussion of hydration to broaden. Discussions of hydration are a tricky subject with some of these crystals, as a few have remarkably long metal-water distances. It is clear that the water molecules associated with these chelates are interacting with the metal ion, otherwise they would not be located proximally in the crystal structure. But, how do we define a "bound water"? How close to the metal ion does the water molecule need to reside for it to be considered "bound"? These questions are impossible to answer definitely. As a result we make the choice to describe the "bound" water as the apically located water in the following data analysis; if the complex has an apically located water molecule it is considered to be $q=1$. For our purposes this makes $q$ a dichotic parameter: there is either an apically located water molecule or there is not. We will account for a spectrum of hydration by considering both the value $q$ and the distance $r$ between the apical water and the lanthanide ion.

In our survey of the literature crystals those chelates with a $\mathrm{d} / \mathrm{c}$ ratio above 0.66 are indisputably hydrated and those with a d/c ratio below 0.64 are indisputably not hydrated. The chelates with a $d / c$ ratio in between these two values (close to 0.65 ) seem to have some ambiguity in their hydration states. In Figure 2.4 there are two crystals which are at or near this 0.65 line and also have apically associated water molecules $(q=1)$ with notably long $r_{\text {LnO }}$ distances $(3.171$ and $2.819 \AA) .{ }^{86,89}$. These structures, with their long $r_{\mathrm{LnO}}$ distances, push the boundaries of what can reasonably be considered as "bound." This supports our initial arbitrarily drawn line at $\mathrm{d} / \mathrm{c}=0.65$ for determining the hydration of LnDOTA-type chelates. 
In this survey there are few complete sets of crystals for one ligand type across the lanthanide series. Of those few complete crystal sets there is no single carboxylate complex that remains in TSAP geometry across the lanthanide series. However, there are a variety of different phosphinate and phosphonate complexes that stay in TSAP geometry across the series and show hydrated species early in the series and dehydrated species late in the series; these chelates have complicated relationships with hydration due to steric crowding and second sphere effects. To be more applicable to the water exchange of clinical contrast agents, we are more interested in what happens in complexes with acetate donors.

Crystal structures of lanthanide chelates with acetate donor ligands either remain entirely in SAP geometry or alternate between SAP and TSAP geometries across the series. For example, the LnDOTA system has varying geometric isomer preferences in solution and in the crystal state depending on the size of the metal in the complex. ${ }^{90}$ The LnDOTA crystals generally reflect the solutions state preferences: in the early series the crystals typically exist as hydrated TSAP isomers, in the mid-series a preference for the hydrated SAP geometry emerges, and after the slight reversal of trend in the solution state allowing a re-emergence of TSAP geometry in the late series, the LnDOTA chelates crystallize as dehydrated TSAP isomers. It has been suggested that the TSAP species early in the series are generally hydrated, while the TSAP species present in the late series are predominately dehydrated. ${ }^{62}$

We are interested in crystal structures of LnDOTA-type chelates with carboxylate donors that remain in TSAP geometry across the series. The preference of the TSAP geometry to 
go from $\mathrm{CN}=9$ to $\mathrm{CN}=8$ with ionic contraction gives rise to the expectation that a single set of complexes that crystallizes in TSAP geometry across the series would show this dehydration process and provide a clear picture of the structural changes associated with dehydration.

Substitutions of the $\alpha$-carbon of the pendant arm in LnDOTA-type complexes have been shown to promote TSAP as the major species in solution. ${ }^{73,}{ }^{75}$ It is often true that the major species in the solution state, being the lower energy species, is the one found in the unit cell of LnDOTA-type chelates. However, this is not always true; the LnTCE-DOTA complexes are an example of this. ${ }^{73,74}$ Due to the ethylene-carboxylate substitutions of the $\alpha$-carbon on the pendant arm, these complexes prefer TSAP geometry in solution. However, in the three published crystal structures $\left(\mathrm{Eu}^{3+}, \mathrm{Gd}^{3+}\right.$, and $\left.\mathrm{Tb}^{3+}\right)$ the chelates are all found in SAP geometry (Figure 2.6).

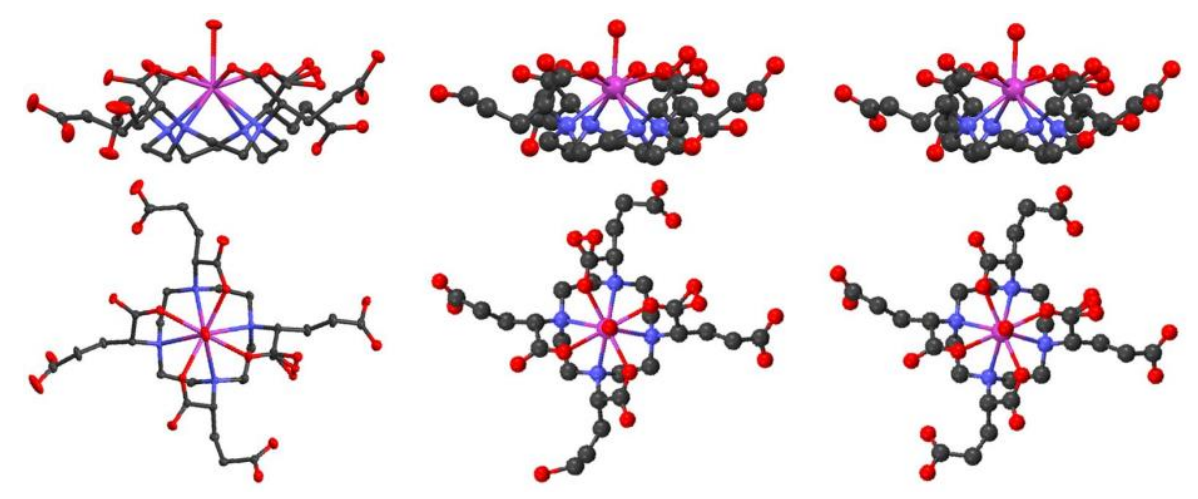

Figure 2.6: $\mathrm{Eu}^{3+}, \mathrm{Gd}^{3+}, \mathrm{Tb}^{3+}$ chelates of TCE-DOTA (left to right) crystal structures, all showing SAP geometry.

In the LnDOTMA system the methyl substitution of the $\alpha$-carbon on the pendant arm allows for the TSAP geometry to be the predominating species across the series in 
solution. While there is a slight increase in the relative abundance of the SAP geometry in the mid-series in solution (reaching a maximum of approximately $30 \%$ at TbDOTMA), it never becomes the dominant species. ${ }^{63}$ LnDOTMA was first reported by Brittan and Desreux in 1984, although it was little remarked upon at the time. ${ }^{59}$ It was over a decade after this initial publication until published research on chelation and characterization of LnDOTMA complexes was seen. ${ }^{71,73,75,76,91,92}$ It was nearly three decades after the first publication that the first crystal structure of these chelates was published.$^{63}$ The published crystal structure of GdDOTMA by Woods et al. had a facile crystallization process and was found to have TSAP geometry, and we hoped that this would be true of the corresponding early and late $\mathrm{Ln}^{3+}$ chelates of DOTMA. ${ }^{63}$

\subsection{Structural variation in LnDOTMA crystals}

The DOTMA chelates of the following $\mathrm{Ln}^{3+}$ ions were prepared: $\mathrm{Ce}^{3+}, \mathrm{Pr}^{3+}, \mathrm{Nd}^{3+}, \mathrm{Sm}^{3+}$, $\mathrm{Eu}^{3+}, \mathrm{Gd}^{3+}, \mathrm{Tb}^{3+}, \mathrm{Dy}^{3+}, \mathrm{Ho}^{3+}, \mathrm{Er}^{3+}, \mathrm{Tm}^{3+}$, and $\mathrm{Yb}^{3+}$. All of our LnDOTMA chelates were prepared from the respective lanthanide trichloride in water at $\mathrm{pH} 5.5 .{ }^{63,93}$ Chelation was confirmed in each case (except $\mathrm{Gd}^{3+}$ ) by ${ }^{1} \mathrm{H}$ NMR spectroscopy. X-ray quality crystals were grown from aqueous solution ( $\mathrm{pH} \sim 5.5$ ) through either slow evaporation of solvent or slow diffusion of ethanol into the mother liquor. Crystallographic data for the chelates of $\mathrm{Pr}^{3+}, \mathrm{Eu}^{3+}, \mathrm{Tb}^{3+}, \mathrm{Dy}^{3+}, \mathrm{Ho}^{3+}, \mathrm{Er}^{3+}$, and $\mathrm{Tm}^{3+}$ were collected and solved at the University of Portland by Prof. Valente. The crystallographic data for the chelates of $\mathrm{Ce}^{3+}, \mathrm{Nd}^{3+}, \mathrm{Sm}^{3+}, \mathrm{Gd}^{3+}, \mathrm{Tm}^{3+}$ and $\mathrm{Yb}^{3+}$ were collected and solved at the University of Minnesota by Prof. Young and his group. 


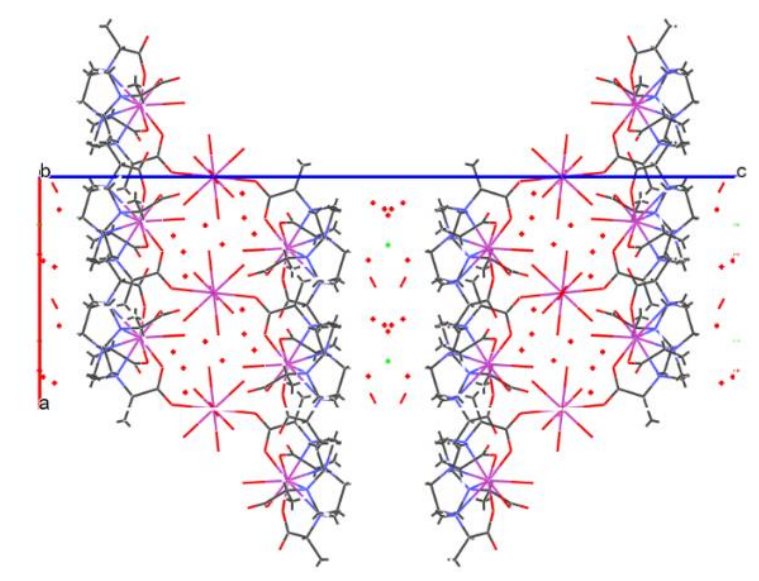

Figure 2.7: The parallel polar sheets typical of LnDOTMA crystals.

Seen here is the PrDOTMA crystal looking down the $b$ axis.

As is common for LnDOTA-type crystal structures, the LnDOTMA complexes form polar sheets with translation relatives (Figure 2.7). In solution the LnDOTMA complexes have time averaged $\mathrm{C}_{4}$ symmetry, while the symmetry observed in the crystal structures is pseudo four-fold geometry with the axes going through the center of the complex (along the complexed $\mathrm{Ln}^{3+}$ ion and apically located water, if present). The reduction of symmetry in the crystal is easily explained by the absence of fluxional effects. Hydrated chloride ions are present in all of the LnDOTMA crystals. To charge balance the anionic chelates and chloride ions, cations in the form of hydrated metal ions (either $\left[\mathrm{Ln}\left(\mathrm{H}_{2} \mathrm{O}\right)_{n}\right]^{3+}$ or $\left.\left[\mathrm{Na}\left(\mathrm{H}_{2} \mathrm{O}\right)_{n}\right]^{+}\right)$are located on the opposite the side of the chelate from the chloride ion. The hydration channels between the sheets of complexes contain disordered water with either the hydrated cations or chloride ions. The hydrated cations are found in the channel between the negatively charged carboxylate groups, while the hydrated anions are found in the channel located between the nitrogen-sides of the complexes. 


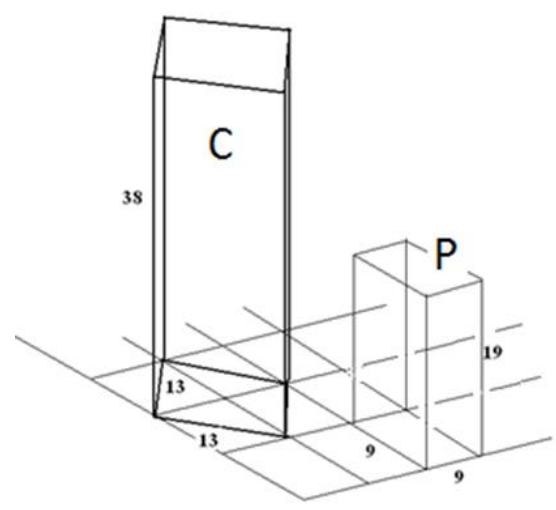

Figure 2.8: Relationship between the side-centered orthorhombic cell (C) and the primitive cell $(\mathrm{P})$.

The resulting basic unit and chelate structure seem relatively unaffected by the identity of the counter cation. The $\mathrm{Pr}^{3+}, \mathrm{Nd}^{3+}, \mathrm{Sm}^{3+}$, and DyDOTMA crystals have hydrated $\mathrm{Ln}^{3+}$ ions between translation sheets. $\mathrm{Ce}^{3+}, \mathrm{Eu}^{3+}, \mathrm{Gd}^{3+}, \mathrm{Ho}^{3+}, \mathrm{Er}^{3+}, \mathrm{Tm}^{3+}$, and YbDOTMA crystals show hydrated $\mathrm{Na}^{+}$ions instead of $\mathrm{Ln}^{3+}$ ions. ${ }^{63}$ TbDOTMA has both $\mathrm{Na}^{+}$and $\mathrm{Tb}^{3+}$ ions in the hydration channel. Despite this difference in hydrated cation species, the structures of the chelates in these crystals are similar and readily compared.

LnDOTMA crystals form a family of related structures with common packing features (Table 2.2). The basic cell is a side-centered orthorhombic parallelepiped $(13 \times 13 \times 38 \AA$ - the volume varies with hydration) with 8 complexes in the unit cell (space group C2221), see Figure 2.8. Derived from this basic cell are three possible primitive pseudo-orthorhombic sub-cells. One sub-cell is $9.5 \times 9.5 \times 19 \AA$, and typically mono- or tri-clinic (P121 or P1), with only 2 complexes in the unit cell. The second subcell is of similar dimensions and volume, but with monoclinic (P112 $)$ symmetry. The third sub-cell is $13 \times 13 \times 19 \AA$, and mono- or tri-clinic (P112 or P1), with 4 complexes 
in the unit cell. The unit cells of LnDOTMA structures can be viewed down any of the three axial directions of the orthorhombic parallelepiped crystal. The difference in these axial views for the LnDOTMA crystals can be seen in Figure 2.9. 


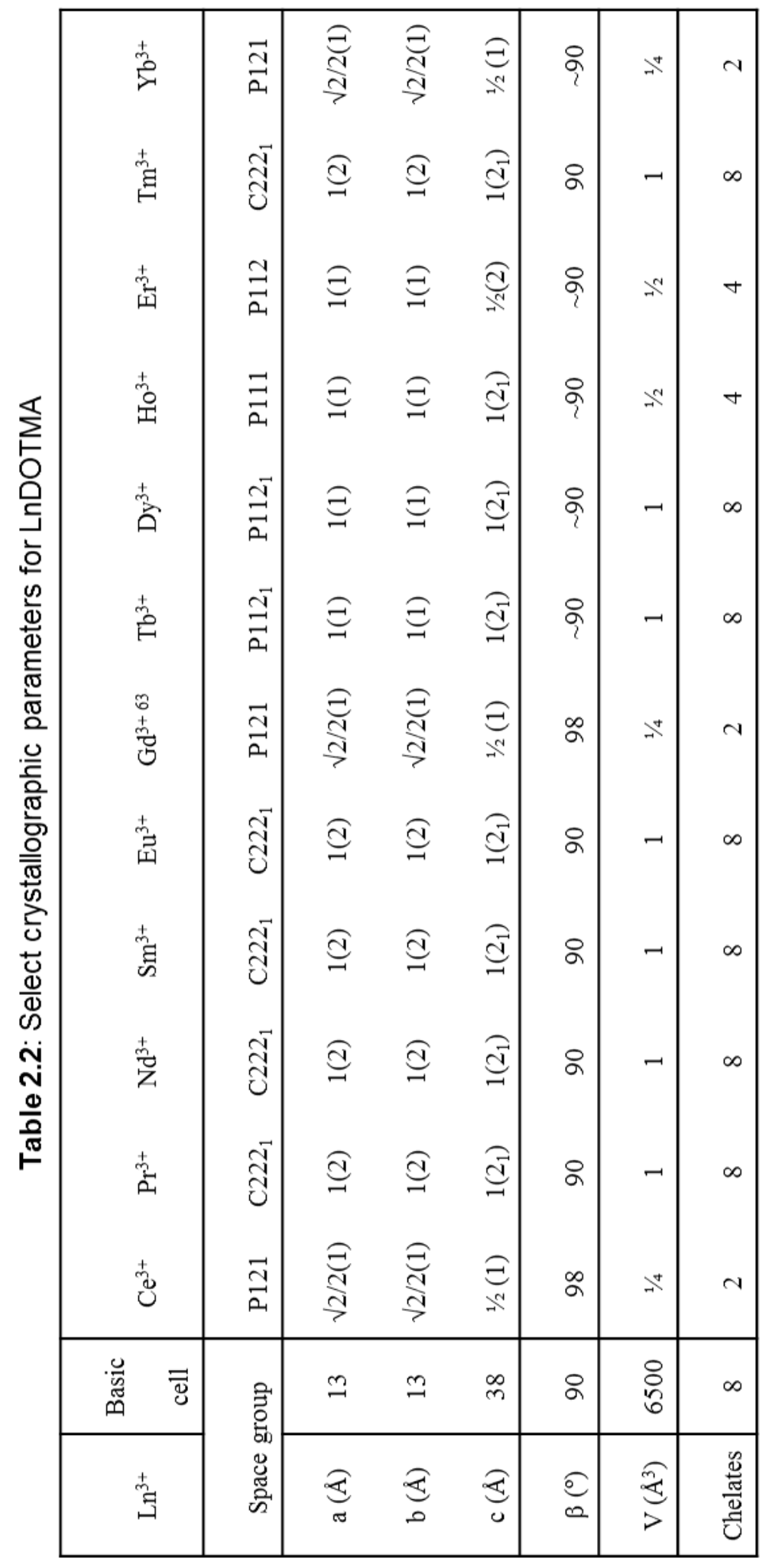




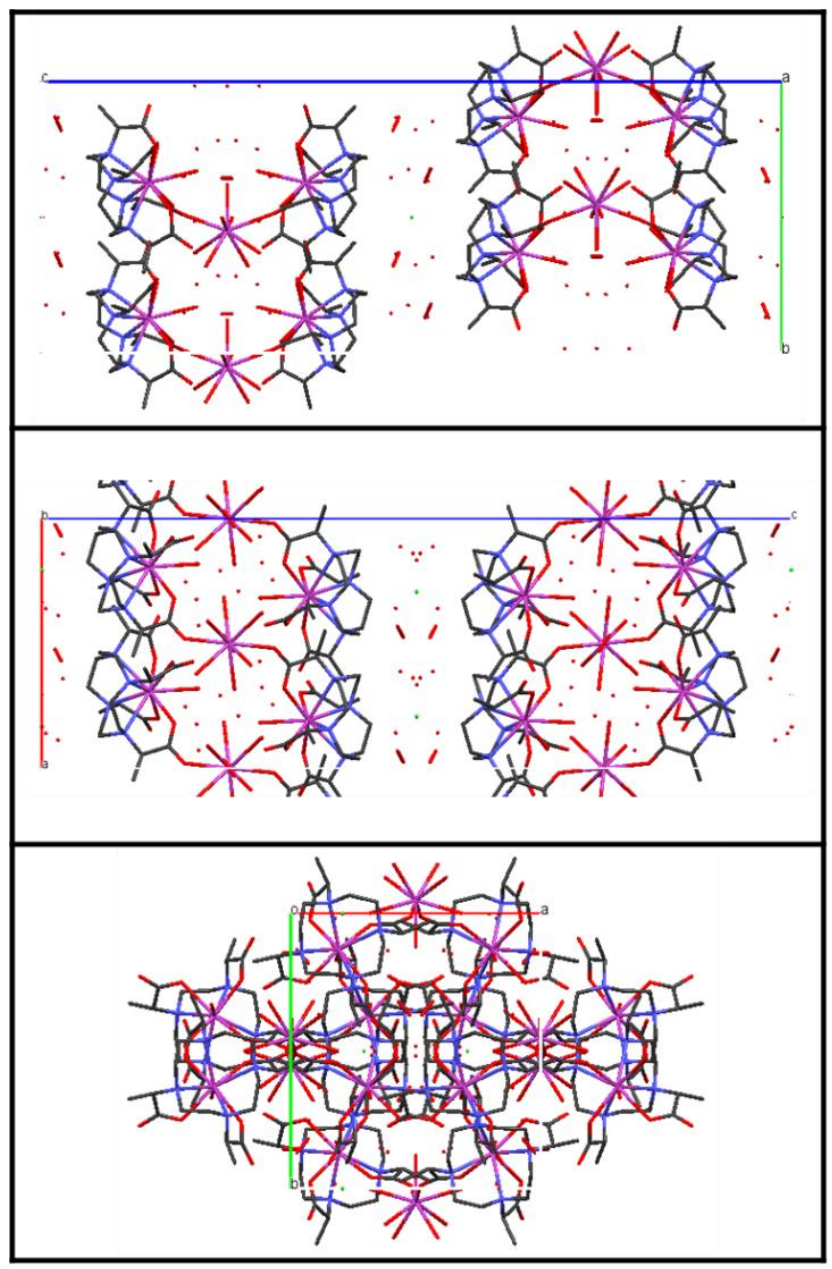

Figure 2.9: Crystal packing of PrDOTMA viewed from the three cell axes, hydrogens excluded for clarity.

Despite the similarities in the larger framework of all the unit cells for LnDOTMA crystals, the complexes of the crystals have clear differences across the series. It is in the trends across the series that the minor structural conformation changes associated with the loss of water can be seen. In order to do a complete analysis of these structures, additional parameters to the water-metal distance and the $d / c$ ratio were surveyed in these complexes. These include the $\mathrm{O}_{4}$ plane area and the chelate, as well as the arm, torsion 
angles (Figure 2.10). ${ }^{19,60,82}$ The $\mathrm{O}_{4}$ plane area is defined by the coordinated oxygens and the area inside them. The relative positions of the coordinated oxygens influence the ability of the apical water to approach the $\mathrm{Ln}^{3+}$ ion and smaller areas can be representative of loss of hydration in LnDOTA-type chelates. The chelate and arm torsion angles are related by geometry. The arm torsion is defined by the twist in the $\mathrm{N}-\mathrm{C}-\mathrm{C}-\mathrm{O}$ bonds, and the chelate torsion is defined by the twist between $\mathrm{O}_{4}$ and $\mathrm{N}_{4}$ planes. There is potential for the chelate torsion angle to be representative of the relative energy differences between SAP and TSAP geometries.

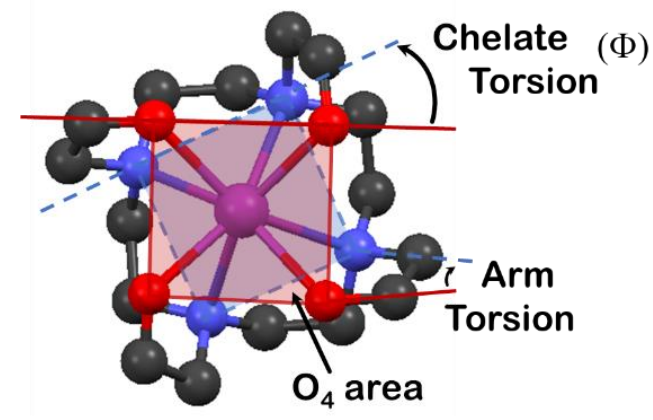

Figure 2.10: The additional crystal parameters used in our analysis.

The discussion of these structures is divided between the early and late series at GdDOTMA. Prior to GdDOTMA all the complexes in each unit cell are identical, while after there exists more than one conformation for the chelate in some unit cells of the LnDOTMA crystals. The hydration of the crystals increases from CeDOTMA to GdDOTMA, then decreases to the dehydrated YbDOTMA. This follows what the literature occasionally refers to as the gadolinium break. The so-called gadolinium break is commonly referenced when the complexes of the early and late lanthanides display different properties. ${ }^{65,94}$ 


\subsection{The early LnDOTMA series}

At first glance the chelate structures of the early lanthanides all look very similar: all chelates within a unit cell are identical, all are hydrated, and all have TSAP geometry (Figure 2.11). However, upon closer inspection there are minor differences that distinguish the chelate conformations of the various LnDOTMA crystal structures.

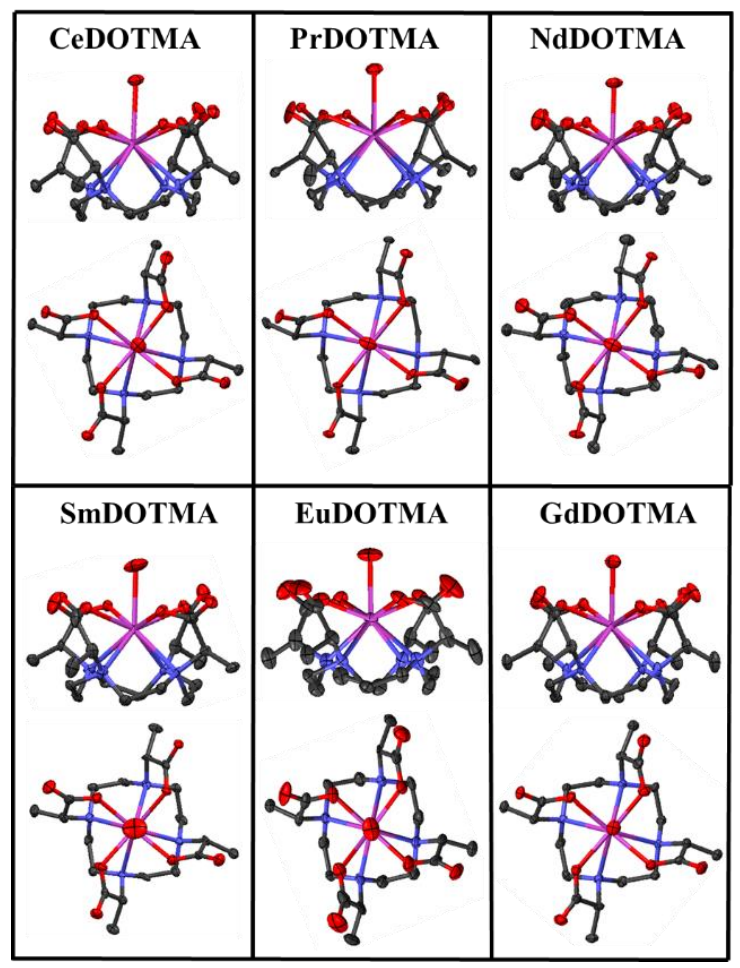

Figure 2.11: Discrete chelates of the $\mathrm{Ce}^{3+}, \mathrm{Pr}^{3+}, \mathrm{Nd}^{3+}, \mathrm{Sm}^{3+}, \mathrm{Eu}^{3+}$, and GdDOTMA unit cells. The hydrogen atoms, counter ions, and water molecules not in the apical positions are omitted for clarity.

Similar to the LnDOTA chelates, there is a general trend for a decrease in $r_{\mathrm{LnO}}$ distances across the early $\mathrm{Ln}^{3+}$ chelates of DOTMA. The $r_{\mathrm{LnO}}$ distance starts with $2.565 \AA$ in CeDOTMA and moves to $2.500 \AA$ in GdDOTMA (Figure 2.12). While in the longer 
range for Ln-water bonds, these $r_{\mathrm{LnO}}$ distances of LnDOTMA fall inside the "normal" range of metal-water bond distances that were determined from the lanthanide nona-aqua ions. ${ }^{33}$

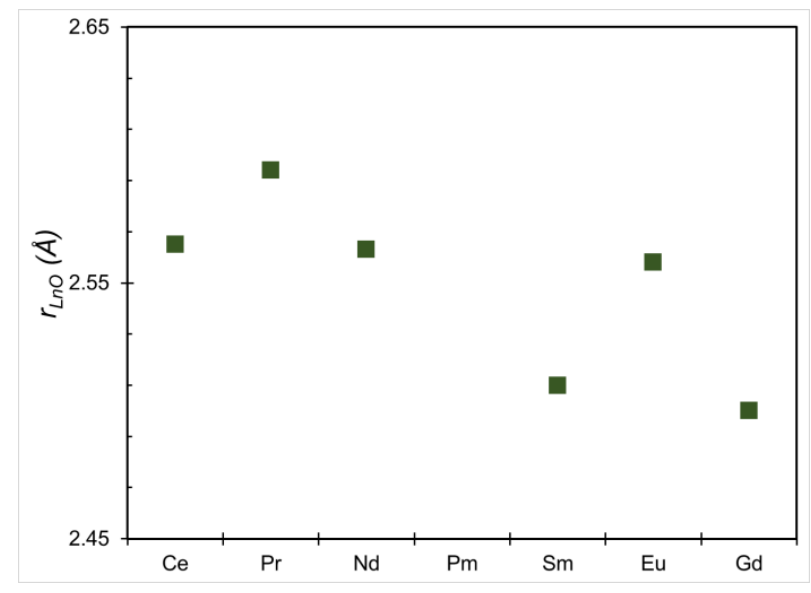

Figure 2.12: Metal-water $\left(r_{\mathrm{LnO}}\right)$ distances for the crystals of early $\operatorname{Ln}^{3+}$ chelates of DOTMA.

It is not only the apically positioned water molecule of the LnDOTMA chelates that is pulled closer to the $\mathrm{Ln}^{3+}$ ion with contraction of the early series, as nuclear pull increases with ionic contraction across the series, all the coordinated donor atoms are pulled tighter to the ion (Figure 2.13). 


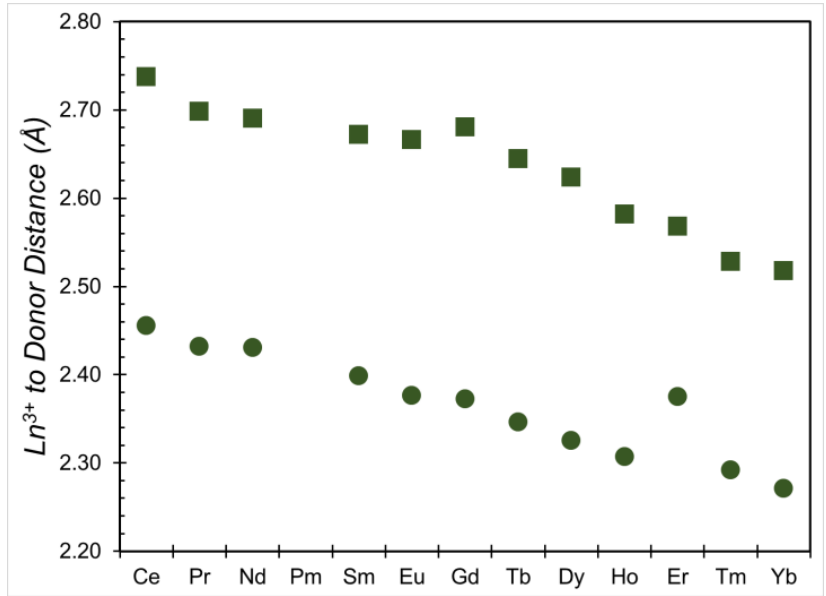

Figure 2.13: The influence of $\mathrm{Ln}^{3+}$ ion contraction on the distances between the metal ion and the donor atom (carboxylate oxygen = circle; nitrogen = square) for the crystals of LnDOTMA. (Best-fit linear slopes of the Ln-N bonds, the Ln-O bonds and $\mathrm{Ln}^{3+}$ size: $\left.\mathrm{m}=-0.017,-0.014,-0.013\right)$

The rate of ionic contraction is similar to the rate of the Ln-O bond contraction across the series. However, the rate of the Ln-N bond contraction is faster than the contraction of metal ion across the series. This is indicative of a decrease in the $\mathrm{d} / \mathrm{c}$ ratio (Figure 2.14). Some of this is attributable to the decrease in ionic size (the smaller ions fit better into the macrocycle); some of this is attributable to increased nuclear pull of the smaller ions (the rate of the $\mathrm{Ln}^{3+}$-donor contraction is faster than the ionic contraction). 


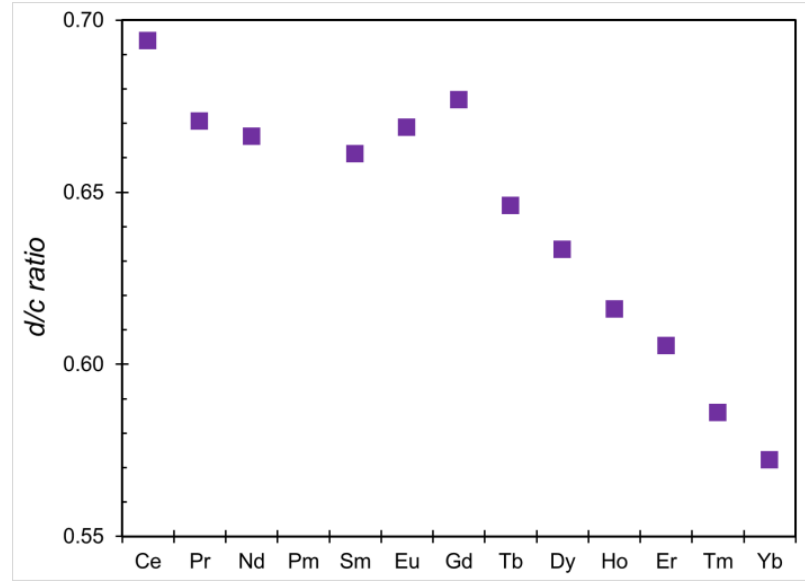

Figure 2.14: $d / c$ ratio for the crystals of early $\mathrm{Ln}^{3+}$ chelates of DOTMA.

The drop of the lanthanide in the coordination cage, despite the increase in hydration from CeDOTMA to GdDOTMA, is notable. The expectation developed from the Lukeš and Hermann structure is that an increase in hydration would raise the lanthanide in the cage. That this is not seen in the early LnDOTMA chelates is explained by the change in size and the associated contraction of the donor atoms of the coordination cage. In addition, as the $\mathrm{Ln}^{3+}$ ions contract, their point of dehydration (moving from $\mathrm{CN}=9$ to $\mathrm{CN}=8$ ) is likely to change; the steric crowding of the water binding site that determines the capacity of the chelate for hydration is dependent on the size of the ion. The common crystal structure parameters and our structural parameters for these early LnDOTMA complexes can be found in Table 2.3. 
CHAPTER 2

Table 2.3: Select bond lengths and angles for LnDOTMA in the early series

\begin{tabular}{|c|c|c|c|c|c|c|}
\hline $\mathrm{Ln}^{3+}$ & $\mathrm{Ce}^{3+}$ & $\operatorname{Pr}^{3+}$ & $\mathrm{Nd}^{3+}$ & $\mathrm{Sm}^{3+}$ & $\mathrm{Eu}^{3+}$ & $\mathrm{Gd}^{3+}$ \\
\hline Ln-O $(\AA)$ & 2.454 & 2.394 & 2.407 & 2.411 & 2.395 & 2.373 \\
\hline Ln-O' $(\AA)$ & 2.477 & 2.461 & 2.463 & 2.361 & 2.347 & 2.358 \\
\hline Ln-O" (§) & 2.454 & 2.446 & 2.436 & 2.426 & 2.402 & 2.391 \\
\hline Ln-O"' (丹) & 2.437 & 2.428 & 2.416 & 2.396 & 2.362 & 2.369 \\
\hline Ln-Q $(O)(\AA)$ & 0.758 & 0.817 & 0.827 & 0.837 & 0.809 & 0.794 \\
\hline$r_{\mathrm{LnO}}(\AA)$ & 2.565 & 2.594 & 2.478 & 2.510 & 2.558 & 2.500 \\
\hline $\mathrm{O}_{4}$ Area $\left(\AA^{2}\right)$ & 10.906 & 10.484 & 10.438 & 10.099 & 9.937 & 10.149 \\
\hline Ln-N $(\AA)$ & 2.765 & 2.683 & 2.703 & 2.673 & 2.732 & 2.704 \\
\hline Ln-N' (̊) & 2.719 & 2.728 & 2.690 & 2.661 & 2.724 & 2.686 \\
\hline Ln-N" (§) & 2.740 & 2.681 & 2.696 & 2.668 & 2.582 & 2.659 \\
\hline Ln-N"' (§) & 2.725 & 2.700 & 2.673 & 2.687 & 2.626 & 2.674 \\
\hline $\operatorname{Ln}-\mathrm{Q}(\mathrm{N})(\AA)$ & 1.719 & 1.663 & 1.651 & 1.633 & 1.634 & 1.663 \\
\hline $\mathrm{Q}(\mathrm{O})-\mathrm{Ln}-\mathrm{Q}(\mathrm{N})\left(^{\circ}\right)$ & 178.45 & 178.91 & 179.48 & 179.63 & 179.44 & 178.40 \\
\hline Chelate torsion angle $\left({ }^{\circ}\right)$ & -25.49 & -25.78 & -25.87 & -26.02 & -26.45 & -26.65 \\
\hline Arm torsion angle $\left(^{\circ}\right)$ & -20.05 & -22.67 & -21.23 & -22.11 & -20.04 & -20.46 \\
\hline $\mathrm{d} / \mathrm{c}$ & 0.69 & 0.67 & 0.67 & 0.66 & 0.67 & 0.68 \\
\hline
\end{tabular}

Where $Q(O)$ and $Q(N)$ are the centroids for the $\mathrm{O}_{4}$ and $\mathrm{N}_{4}$ planes. 


\subsection{The late LnDOTMA series}

The basic cell organization of the LnDOTMA crystals in the second half of the series is similar to that seen in the early series: all the LnDOTMA chelates in the second half of the lanthanide series have TSAP geometry (Figure 2.15). Some of the late series crystals are found to have more than one unique chelate structure in the unit cell. This is a result of contraction across the lanthanide series. As the $\mathrm{Ln}^{3+}$ ion becomes smaller the apically located water is pushed out of the coordination sphere of the lanthanide as the chelate goes from $\mathrm{CN}=9$ to $\mathrm{CN}=8$. This change in hydration is not observed in the crystal structures of LnDOTA since those chelates generally crystallize in hydrated forms: the early lanthanides as TSAP isomers and the late lanthanides as SAP isomers. 


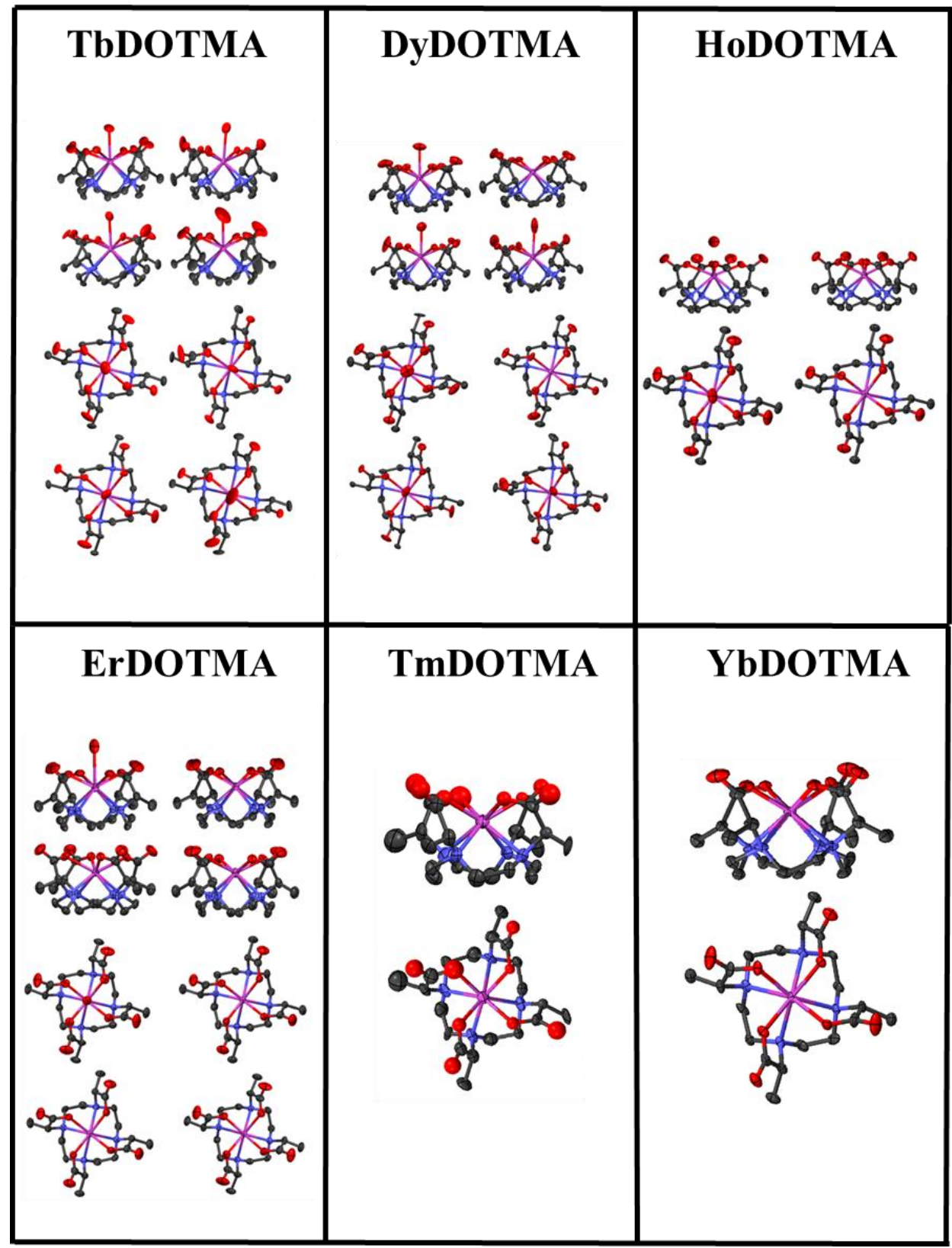

Figure 2.15: Discrete chelates of $\mathrm{Tb}^{3+}, \mathrm{Dy}^{3+}, \mathrm{Ho}^{3+}, \mathrm{Er}^{3+}, \mathrm{Tm}^{3+}$, and YbDOTMA unit cells.

The hydrogen atoms, counter ions, and water molecules not in the apical positions are omitted for clarity.

The division of the early and late LnDOTMA series is based on the gadolinium break. Before this break the apical water is drawn closer to the lanthanide ion but is pushed 
away after the break (Figure 2.16), with GdDOTMA showing the shortest $r_{\text {Lno }}$ distance. The first crystal structure in the LnDOTMA series to show more than one unique structure in the unit cell is TbDOTMA, directly to the right of $\mathrm{Gd}^{3+}$ in the series. The TbDOTMA crystal has four unique chelates in the unit cell; each has an apically located water molecule, but all the $r_{\mathrm{LnO}}$ distances are different (ranging from $2.572-2.637 \AA$ ). The average of the $r_{\mathrm{LnO}}$ distances for TbDOTMA is longer than the $r_{\mathrm{LnO}}$ distance seen in GdDOTMA (Tables 2.3 and 2.4). The $r_{\mathrm{LnO}}$ distances continue to increase with each step to the right in the series. One might argue that the impact of a $0.16 \AA$ difference in $r_{\mathrm{LnO}}$ (as between the $\mathrm{Gd}^{3+}$ and $\mathrm{Ho}^{3+}$ chelates of DOTMA) is not significant. However, relaxivity is dependent on $r^{-6}$; this means that even small changes of $0.15 \AA$ in $r_{\mathrm{GdH}}$ distances can have a large (16\% at low fields) impact on relaxivity. ${ }^{50}$

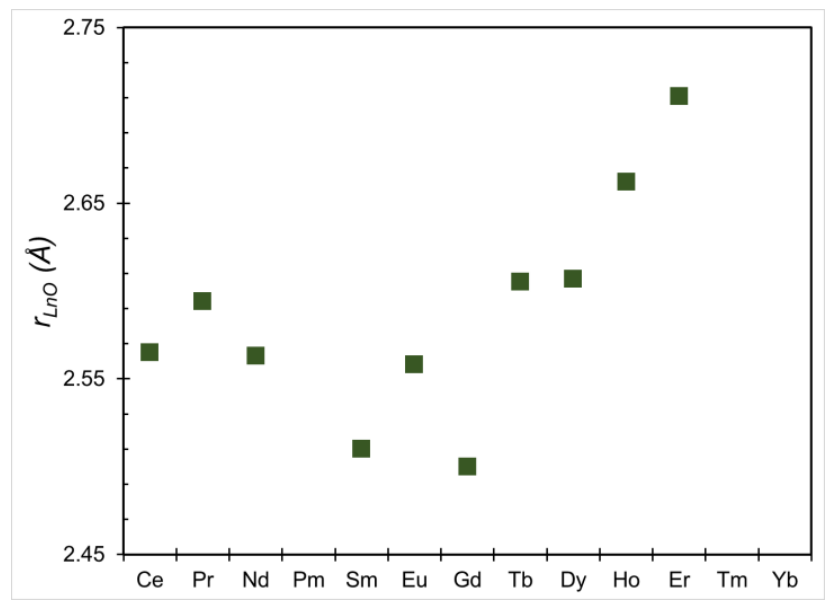

Figure 2.16: Metal-water $\left(r_{\llcorner n O}\right)$ distances for the crystals of LnDOTMA.

The DyDOTMA crystal is the first of the series to show a chelate in the unit cell without a water molecule in the apical position "bound" or "unbound" (Figure 2.15). If we consider only the presence of a water molecule in the apical position (regardless of 
binding), the pattern of reducing hydration in LnDOTMA starts after the $\mathrm{Tb}^{3+}$ chelate in the $\mathrm{Dy}^{3+}$ chelate and continues linearly to the fully dehydrated $\mathrm{Tm}^{3+}$ chelate. In the middle of this decreasing series is the $\mathrm{Ho}^{3+}$ chelate with two chelates per unit cell, yet only one possesses an apical water molecule.

If the distance of the apical water from the lanthanide is also taken into account (as it is the dipole-dipole interaction that governs relaxivity), then the drop in hydration starts earlier at $\mathrm{Tb}^{3+}$. GdDOTMA has the shortest $r_{\mathrm{Lno}}$ distance of the series (2.500 $\mathrm{\AA}$ ) affording it the highest hydration state $\left(q / r^{6}\right)$ in the series (Figure 2.16). All four chelates in the $\mathrm{Tb}^{3+}$ unit cell have apical waters, but the water $r_{\mathrm{LnO}}$ distances are long - on average 2.605 $\AA$ (Table 2.4) - and are longer than the $r_{\mathrm{LnO}}$ distance in the $\mathrm{Gd}^{3+}$ chelate. The three DyDOTMA chelates with apical waters also have long $r_{\mathrm{LnO}}$ distances (average of 2.607 $\AA$ ). This feature continues until water is completely displaced at $\mathrm{Tm}^{3+}$ : the single hydrated chelate in the unit cells of the $\mathrm{Ho}^{3+}$ and $\mathrm{Er}^{3+}$ chelates have distances of $2.662 \AA$ and $2.711 \AA$ A, respectively (Figure 2.16, Table 2.5). Interestingly, even the unambiguously dehydrated TmDOTMA and YbDOTMA crystals show this trend. The $\mathrm{Tm}^{3+}$ chelate has a water molecule located equidistant (5.021 $\mathrm{A}$ ) from both chelates and one of the $\mathrm{Yb}^{3+}$ chelates has a water molecule even further away $(5.228 \AA$ ). These waters are not included in Figure 2.15 because they lie at the extreme end of the range of distances over which dipole-dipole interactions occur. In fact they are not only well beyond an inner sphere hydration position, but they are hydrogen bonded to solvated $\mathrm{Na}^{+}$ions in the unit cell. 


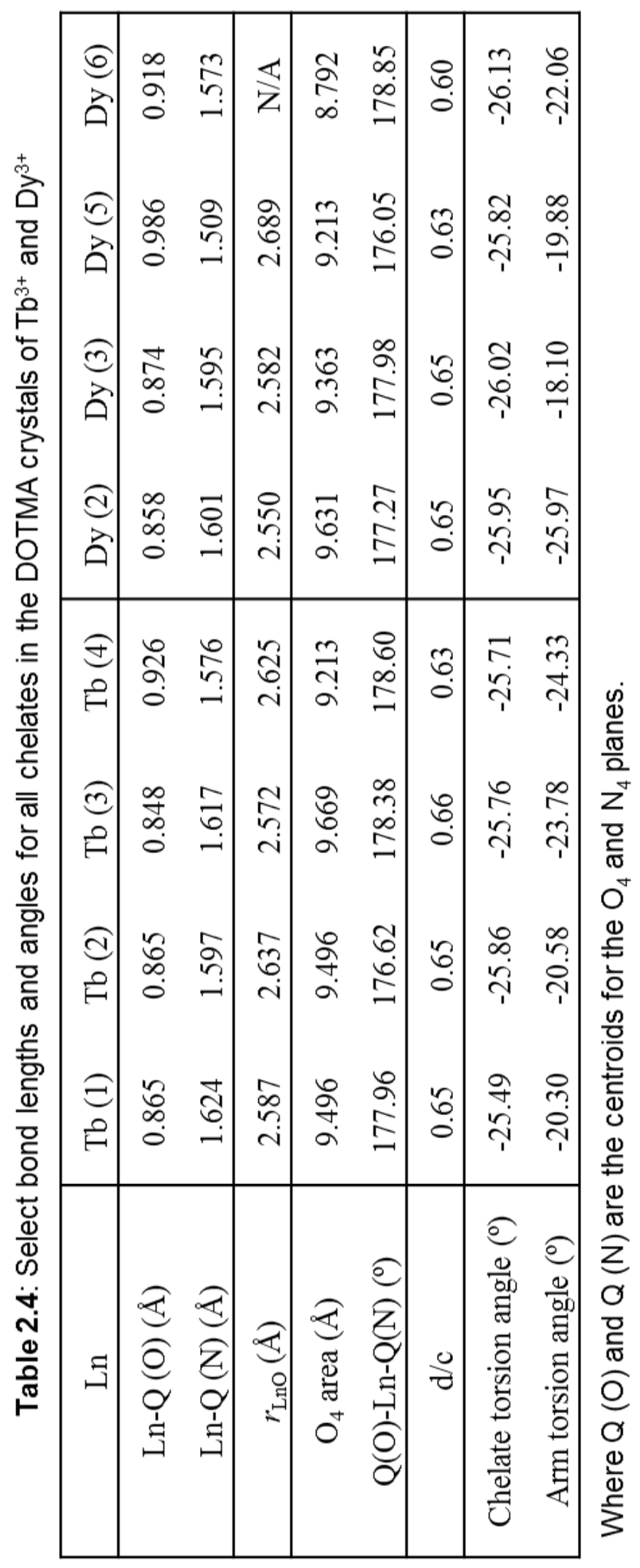




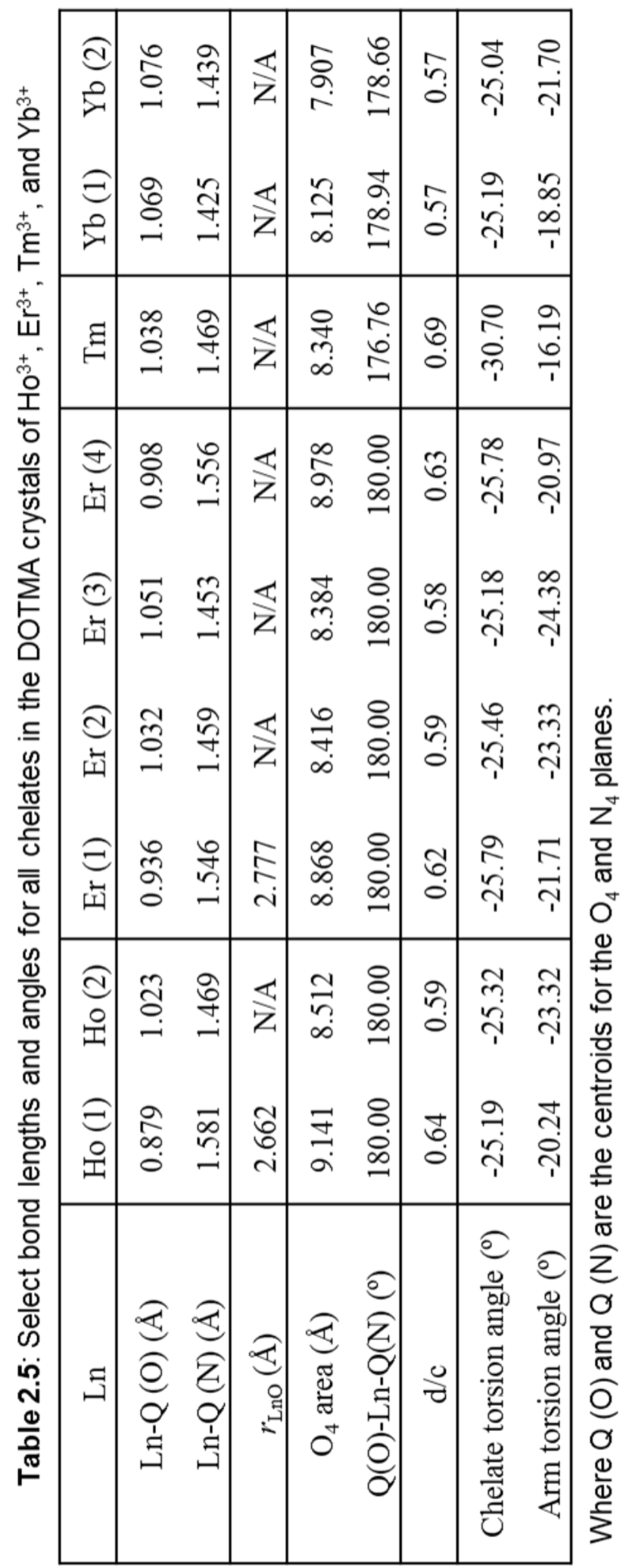




\subsection{The complete LnDOTMA series}

\subsubsection{Considering the series as a whole}

Direct comparison of our LnDOTMA structures that have all crystallized in TSAP geometry allows for a picture of water exchange and the structural changes associated with exchange to be developed. Earlier in the $\mathrm{Ln}^{3+}$ series the chelates of DOTMA all have a water molecule that is unambiguously bound in the apical position and duplicated chelates within the unit cell (Figure 2.13). Later in the series the crystal structures have more than one chelate structure in the unit cell - the number of chelates without a bound water molecule increases by one for each lanthanide from $\mathrm{Tb}^{3+}$ to $\mathrm{Tm}^{3+}$. This results in the unusual occurrence of unit cells that have more than one distinct chelate (Figure 2.15). At the end of the series, when the $\mathrm{Ln}^{3+}$ ion is sufficiently small so as to exclude water in the coordination cage (indisputably $q=0$ chelates), the chelate structures within the crystal are again duplicates or near-duplicates. The changes in coordination geometry that occur on passing from CeDOTMA to YbDOTMA provide insight into the structural changes in these chelates associated with water exchange.

These structural changes are associated with the effect of the ligand accommodating smaller metal ions. Eventually the crowding associated with ionic contraction pushes water off the metal ion completely. Probing these structural changes using solution techniques, such as NMR, is difficult as the structural changes associated with changing between coordination geometries in solution are larger and slower when compared to the relatively small and very rapid structural changes one might see from water exchange. The crystal structures of the LnDOTMA chelates allow us to visualize these small 
conformational changes associated with water exchange in the solid state. These structural changes can be understood by analyzing different structural parameters: $r_{\mathrm{LnO}}$, $\mathrm{O}_{4}$ area, $\mathrm{d} / \mathrm{c}$ ratio, c values, and chelate torsion (Figures 2.3 and 2.10).

Figure 2.17 shows the relationship between the $\mathrm{N}_{4}$ and $\mathrm{O}_{4}$ planes, the position of the metal ion and any apically bound water molecule. This perspective shows that the apical water molecule is initially pulled closer to the lanthanide ion with ionic contraction and then, after the gadolinium break, is pushed away as the lanthanide ion size becomes insufficient to accommodate $\mathrm{CN}=9$. Maintaining the middle of the $\mathrm{Ln}^{3+}$ ion in a consistent position across the series reveals the differences in the position of the ligand cage as the $\mathrm{Ln}^{3+}$ ions contract. These changes also seem to represent the changes that occur in the chelate structure as the bound water molecule comes and goes. As the water molecule dissociates the $\mathrm{Ln}^{3+}$ ion gets closer to the $\mathrm{N}_{4}$ plane and further from the $\mathrm{O}_{4}$ plane. 

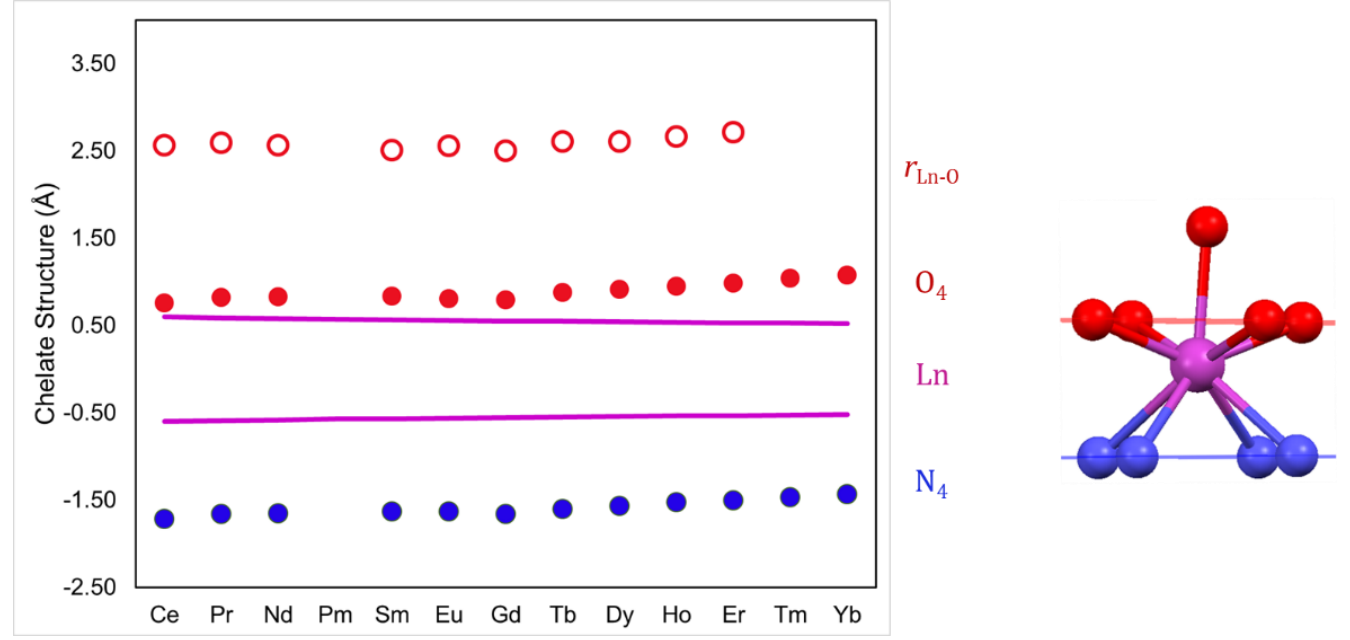

Figure 2.17: A cross-section of the LnDOTMA crystals. By holding the center of the lanthanide steady (purple lines), the distances to the axial water (open red circles), $\mathrm{O}_{4}$ plane (red circles), and $\mathrm{N}_{4}$ plane (blue circles) can be seen.

The changes in ligand conformation as the water molecule is pushed out of the coordination cage across the series of LnDOTMA crystal structures could be interpreted as demonstrating the conformational changes associated with water exchange in solution. With decreased coordination sphere size in the later $\mathrm{Ln}^{3+}$ ions, the balance of hydration moves toward "dehydrated" which resulted in the unusual occurrence of some chelates in a single crystal to have variation in apical water presence (Figure 2.18). 


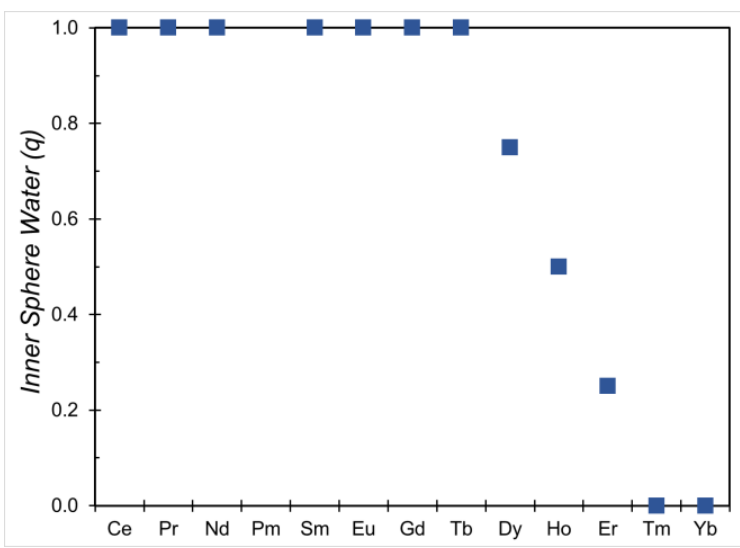

Figure 2.18: The relationship of inner sphere water $(q)$ associated with the chelate as the lanthanide contracts across the series in the LnDOTMA crystals

The presence of an apical water molecule is not a dichotomous description of hydration: water is not either on or off. The distance between the metal and the water must be considered, as it is an integral parameter for the dipole-dipole interaction of proton relaxation by the $\mathrm{Gd}^{3+}$ chelate. The interaction of the inner sphere water with the $\mathrm{Ln}^{3+}$ ion is commonly defined as "bound water," but how far from the metal ion can a water molecule be before it is no longer considered bound? Apical water placement is controlled by electrostatic forces; the proximity of the electron rich carboxylate groups affects how closely the electron rich water oxygen can approach the electron deficient metal ion. The shortest $r_{\mathrm{LnO}}$ distance of the series is found for GdDOTMA (Figure 2.16). The chelates of the later $\mathrm{Ln}^{3+}$ ions that have apically located water molecules show longer water-lanthanide distances that are difficult to consider "bound." However, that there exists an apically located water molecule in these complexes suggests some level of interaction between the lanthanide ion and the water molecule. The long water-lanthanide distances of these late-series LnDOTMA chelates are indicative of a reduction in the 
hydration state $\left(q / r^{6}\right)$ of these molecules, even though water is still present in the apical position (Figure 2.19).

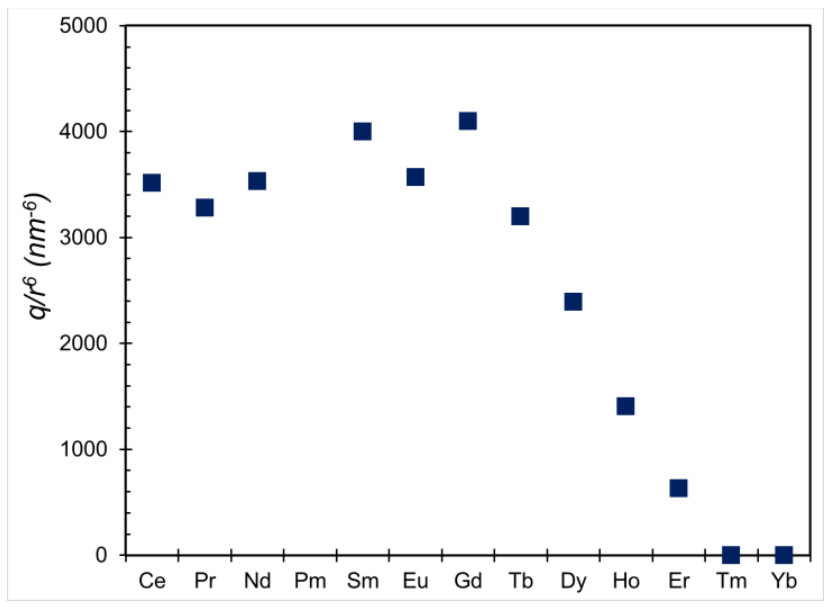

Figure 2.19: The relationship of hydration (considered as a function of $q / r^{6}$ ) with lanthanide contraction in the LnDOTMA crystals.

The way that the lanthanide ion interacts with the solution is imperative to the application of these agents. In the equations describing relaxivity there is no clear difference between one nearby water and two distant waters. For ease of analysis in the LnDOTMA system, we consider all chelates with an apically positioned water molecule within $3 \AA$ to be " $q=1$ " and the hydration of the chelate to be a construct of $q / r^{6}$. By doing this we begin to approach a better theoretical model of hydration, as $q / r^{6}$ this is representative of how these parameters directly impact relaxivity in the SBM equations. With this consideration of hydration GdDOTMA is the most hydrated of the LnDOTMA series (Figure 2.19).

\subsubsection{Other structural changes associated with hydration}

There are conformational changes to the ligand structure associated with the increasing $r_{\mathrm{LnO}}$ distances of the later $\mathrm{Ln}^{3+}$ chelates: tightening of the $\mathrm{O}_{4}$ area, a drop of the 
lanthanide ion in the coordination sphere, and altering twist angles between the $\mathrm{O}_{4}$ and $\mathrm{N}_{4}$ planes. We discuss these conformational changes by starting with the relationship of the apical water to the $\mathrm{Ln}^{3+}$ ion. The distance of the water from the metal ion correlates with the position of the metal ion in the coordination cage. This is attributed to the metal ion seeking more electronic density from the aza-donor atoms, and pulling the oxygen-donor atoms closer together over the lanthanide ion.

The drop of the $\mathrm{Ln}^{3+}$ ion in the coordination cage is represented in a decrease in the $\mathrm{d} / \mathrm{c}$ ratio. There is a steady decrease in the $\mathrm{d} / \mathrm{c}$ ratio across the $\mathrm{Ln}^{3+}$ series for the DOTMA chelates that agrees with the trend seen in other LnDOTA-like chelates (Figure 2.20). The literature survey suggests a division between hydrated and dehydrated complexes at a d/c value of 0.65 (Figure 2.5). The LnDOTMA chelates have a change in hydration around $\mathrm{d} / \mathrm{c}=0.65$, but the chelates of the late series suggest a spectrum of hydration rather than a dividing line (Figure 2.20). This is supported by the mix of hydration in the chelates of the unit cells in the late series LnDOTMA. These chelates suggest that the preliminary idea that a $\mathrm{d} / \mathrm{c}$ ratio of 0.65 might be indicative of apical water dissociation was too simplistic. Just in the two unit cells of the $\mathrm{Tb}^{3+}$ and $\mathrm{Dy}^{3+}$ chelates there are four distinct complexes with $\mathrm{d} / \mathrm{c}$ values of 0.65 - all have different $r_{\mathrm{LnO}}$ values that range from 2.550 to $2.637 \AA$. 


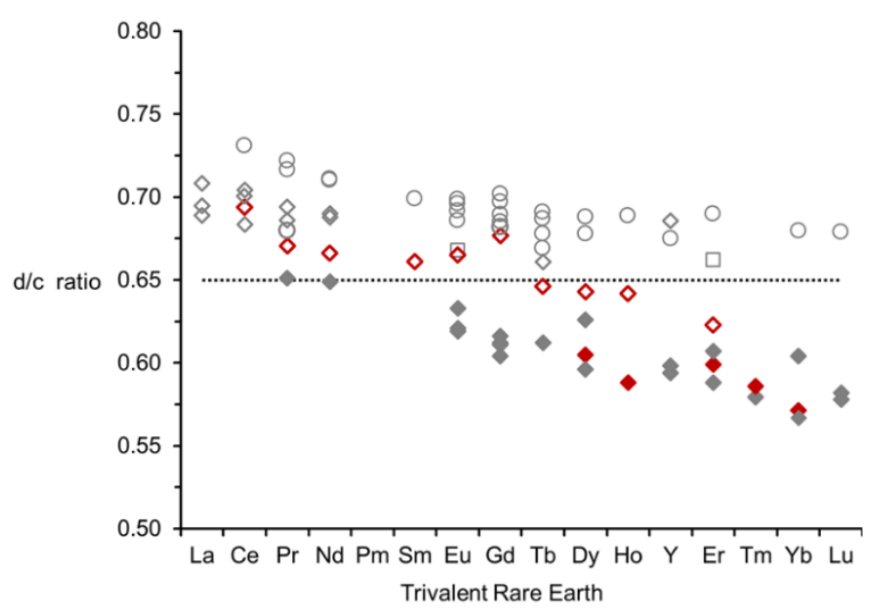

Figure 2.20: $21,27,37,39,40,73,83-89$ Compiled d/c ratios from LnDOTA-type chelates.

Diamonds $=$ TSAP, squares $=$ SAP. Open and closed data points are $q=1$ and 0 , respectively. Gray data represent the literature complexes with pendant arms containing acetates, amides, pyridyls, phosphonates with and without aromatic groups, respectively

(as seen in Figure 2.5). Red data represent the LnDOTMA chelates from this work.

While the $\mathrm{d} / \mathrm{c}$ ratio in the late lanthanides has a close relationship with $r_{\mathrm{LnO}}$ distances, there is some ambiguity in correlating a given $\mathrm{d} / \mathrm{c}$ ratio with specific $r_{\mathrm{LnO}}$ distance: in the late series of LnDOTMA, the $\mathrm{d} / \mathrm{c}$ ratios of the complexes with apical waters ranges from $0.62-0.66$ with corresponding $r_{\mathrm{LnO}}$ distances of $2.711-2.550 \AA$. Using the $\mathrm{d} / \mathrm{c}$ ratio as an indicator of hydration in these late-lanthanide crystals has some imprecision (Figure 2.21). In the $\mathrm{Tb}^{3+}$ cell 4 of 4 chelates have apically located waters and a range of $\mathrm{d} / \mathrm{c}$ ratios of $0.63-0.66$; in the $\mathrm{Dy}^{3+}$ cell 1 of the 4 chelates is missing an apically located water $(d / c=0.60)$ and the 3 chelates with apical waters have a $d / c$ range of $0.63-0.65$; in the $\mathrm{Ho}^{3+}$ cell one of the two has an apically located water $(\mathrm{d} / \mathrm{c}=0.64)$ while the other does not $(\mathrm{d} / \mathrm{c}=0.59)$; and in the $\mathrm{Er}^{3+}$ cell only one has an apical water molecule 
$(d / c=0.62)$ while the other three chelates are dehydrated and have a $d / c$ ratio range of $0.58-0.64$ (Tables 2.4 and 2.5).

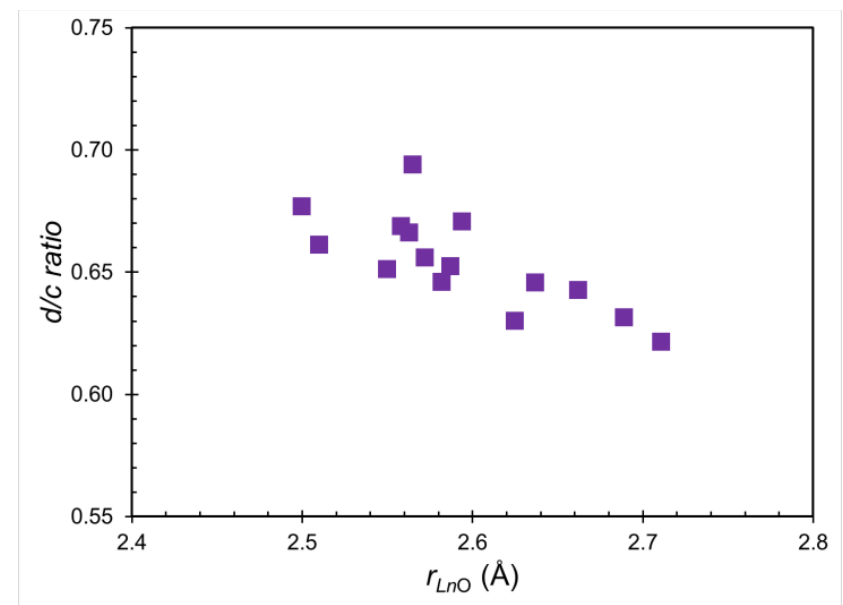

Figure 2.21: The relationship of the $\mathrm{d} / \mathrm{c}$ ratio and apical water distances in the LnDOTMA crystals

It is apparent from our LnDOTMA crystal structures that chelates with $\mathrm{d} / \mathrm{c}<0.6$ do not have an apically located water molecule, but it is difficult to definitively say when dehydration occurs. The $\mathrm{d} / \mathrm{c}$ ratio ranges from 0.69 to 0.64 for the LnDOTMA chelates with an apical water, and from 0.60 to 0.57 for the chelates without an apical water. What we consider the most hydrated LnDOTMA crystal in the series (GdDOTMA) has a d/c ratio of 0.68 and a $r_{\mathrm{LnO}}$ distance of $2.500 \AA$. This results in a spectrum of $\mathrm{d} / \mathrm{c}$ ratios and $r_{\mathrm{LnO}}$ distances between this most hydrated chelate and those chelates without inner sphere waters.

Associated with the decrease in the $\mathrm{d} / \mathrm{c}$ ratio across the series is a change in the position of the coordinating oxygen donors of the $\mathrm{O}_{4}$ plane. These donor atoms pull closer together around the apical position as the $\mathrm{Ln}^{3+}$ ion drops in the coordination cage. This 
movement of the oxygen donors is seen across the lanthanide series (Figure 2.22): the $\mathrm{O}_{4}$ area steadily contracts from $10.9 \AA^{2}$ at $\mathrm{Ce}^{3+}$ to $8.0 \AA^{2}$ at $\mathrm{Tb}^{3+}$.

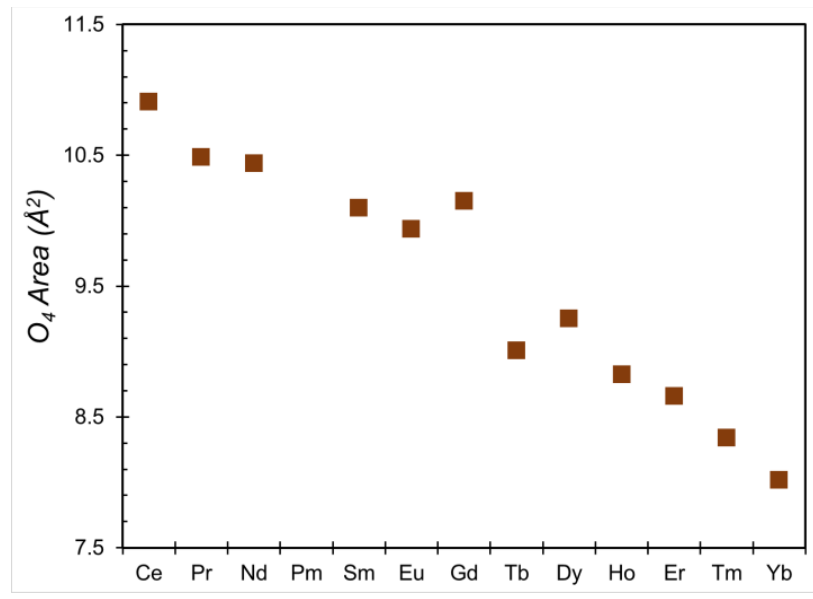

Figure 2.22: Relationship of the $\mathrm{O}_{4}$ area with lanthanide contraction in the LnDOTMA crystals

Interestingly, the height of the $\mathrm{Ln}^{3+}$ coordination cage (the distance between the $\mathrm{O}_{4}$ and $\mathrm{N}_{4}$ planes) is not directly related to the decrease of the $\mathrm{d} / \mathrm{c}$ ratio across the series. The gadolinium break is again visible in this parameter; the height of the coordination cage decreases along the first half of the series and increases across the second half of the series (Figure 2.23). This means that while the metal is moving consistently towards the $\mathrm{N}_{4}$ plane (and away from the $\mathrm{O}_{4}$ plane), the $\mathrm{N}_{4}$ and $\mathrm{O}_{4}$ planes are also moving relative to each other. 


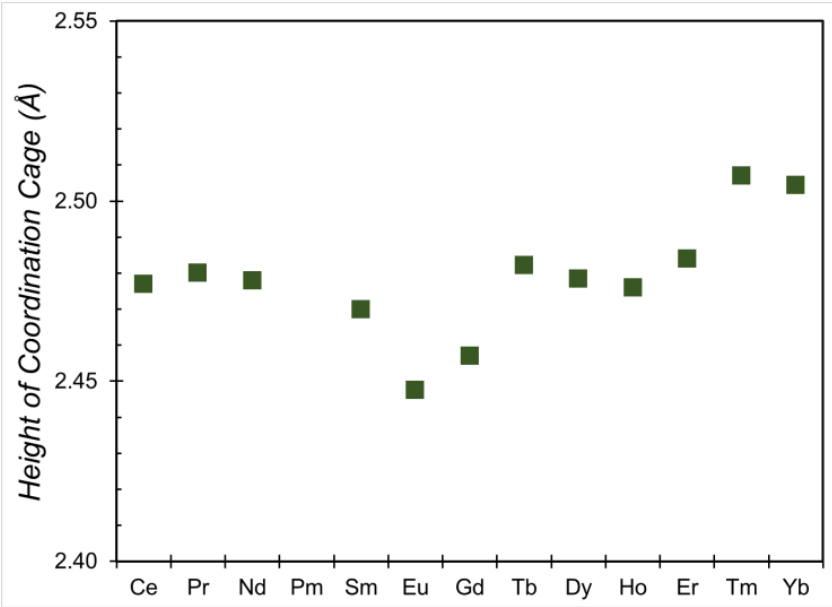

Figure 2.23: The relationship of the coordination cage height with ionic contraction across the series of LnDOTMA chelates.

In order to achieve these structural adjustments to the coordination cage height the ligand structure requires conformational changes to the orientation of the pendant arms (the $\mathrm{N}-\mathrm{C}-\mathrm{C}-\mathrm{O}$ torsion angle) that are reflected in changes in the chelate torsion angles (the twist between the $\mathrm{N}_{4}$ and $\mathrm{O}_{4}$ planes, as defined in Figure 2.10). At the extremes of these torsion angles exist the taller TSAP geometry (where the arms rest near perpendicular to the $\mathrm{N}_{4}$ and $\mathrm{O}_{4}$ planes) and the shorter SAP geometry (where the pendant arms rest in more of a diagonal orientation relative to the $\mathrm{N}_{4}$ and $\mathrm{O}_{4}$ planes). The absolute values of the chelate torsion angles of SAP geometries are larger than those of TSAP geometries. It can be seen in Figure 2.24 that the chelate torsion angle moves toward a conformation closer to SAP geometry for the early $\mathrm{Ln}^{3+}$ chelates, then back toward TSAP geometry in the late $\mathrm{Ln}^{3+}$ chelates. 


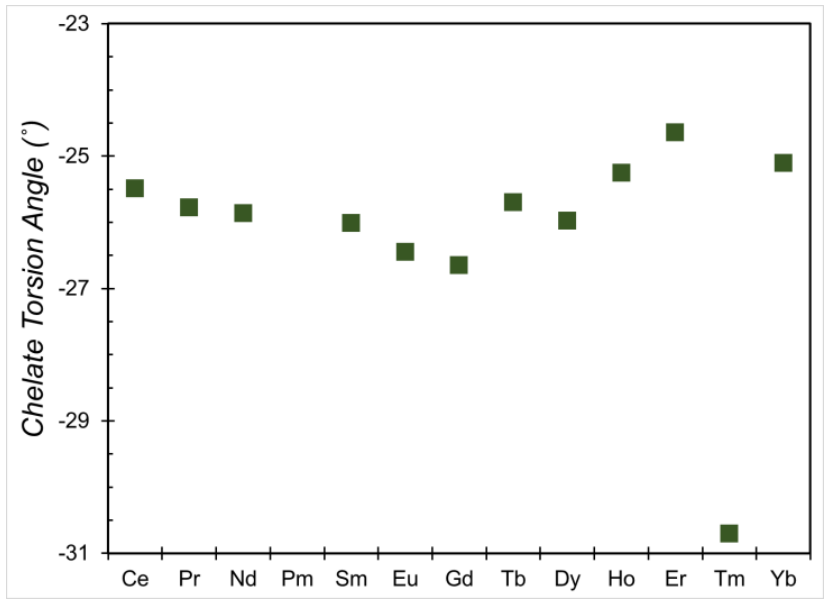

Figure 2.24: The relationship of the average chelate torsion angles with $\mathrm{Ln}^{3+}$ ion contraction in the LnDOTMA crystals.

That the turnaround point for chelate torsion angles occurs near the most hydrated chelate of the series suggests that these angles may have a relationship with the $r_{\mathrm{LnO}}$ distance of the chelate. A close relationship between the chelate torsion angle and the $r_{\mathrm{LnO}}$ distances can be seen in Figure 2.25. This relationship is interesting as it indicates that the hydrated LnDOTMA chelates prefer a larger absolute chelate torsion angle - the extreme of which is SAP geometry - while the dehydrated chelate prefers the smaller absolute chelate torsion angles of TSAP geometry. 


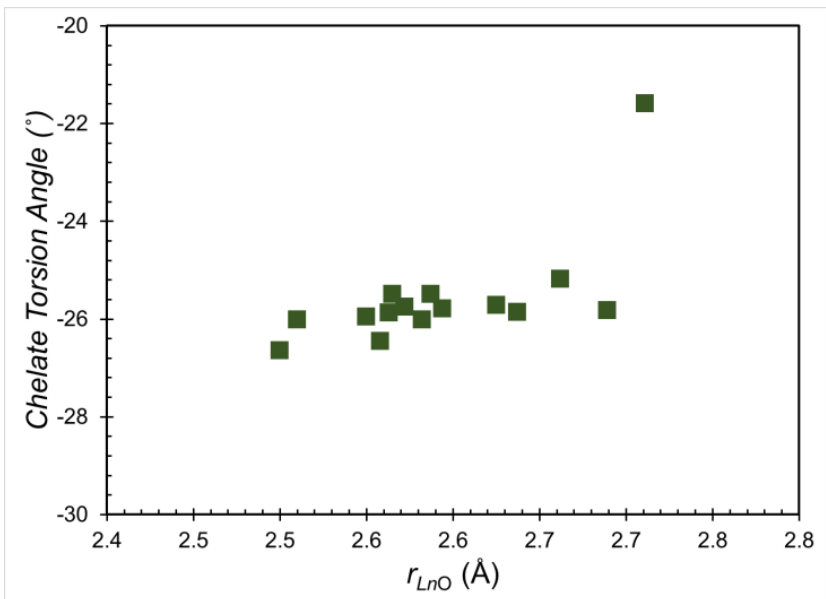

Figure 2.25: The relationship of chelate torsion angles from all $q=1$ chelates in the LnDOTMA crystals with lanthanide-water distances.

The relationship between hydration and chelate torsion angle is not unique to the LnDOTMA chelates. Indeed, the tendency for the absolute value of the chelate torsion angle to decrease (move toward TSAP geometry) with increasing $r_{\text {LnO }}$ distances can be seen in the other LnDOTA-like literature crystal structures with carboxylate and amide donors (Figure 2.26). Both the SAP and TSAP LnDOTA-type chelates in Figure 2.26 move towards a taller coordination cage - indicative of ideal TSAP geometry - as the apical water moves further away from the lanthanide ion. This trend is stronger in chelates with SAP geometry. 


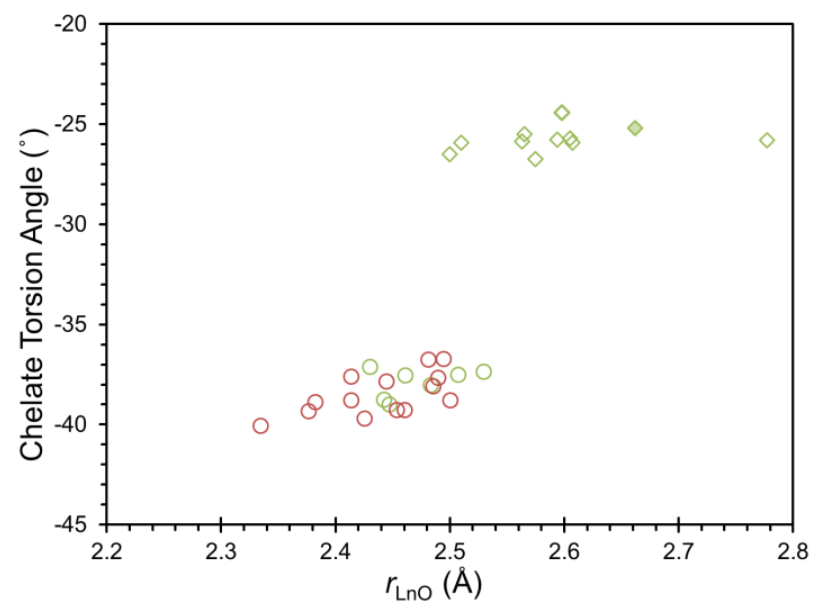

Figure 2.26: The relationship between chelate torsions angles and lanthanide-water distances of the carboxylate (green) and amide (red) donors involved in the previous crystal survey. SAP complexes (circles) show a stronger relationship than the TSAP (diamonds) to apical water distances.

Differences in chelate structure for the hydrated and dehydrated species of a given geometry have been predicted previously; the dehydrated species in solution was determined to have a shorter cage height than the hydrated species in solution for both SAP and TSAP geometries. ${ }^{62}$ This prediction agrees with observations of LnDOTA-type crystal structures: a shorter cage height results from the increase of the chelate torsion.

\subsection{Crystal structure \& solution state of HoDOTMA}

In the LnDOTA system, holmium is positioned where the chelates begin to show preference for dehydration by increasing the population of the TSAP isomer. The crystal structure of HoDOTMA has two chelates in the unit cell: one of which has an apically located water and the other chelate is clearly dehydrated. Having chelates of different hydration in the unit cell is uncommon in the LnDOTA-type derivatives. The structures 
seen in the HoDOTMA crystal support the idea that water exchange in solution is associated with structural motion of the ligand. The chelate with an apical water molecule seems poised midway between a dehydrated structure and the hydrated structure observed for $\mathrm{Gd}^{3+}$ (Figure 2.27). This structure - with its long $\mathrm{Ln}-\mathrm{OH}_{2}$ distance - is inherently different from the Lukeš and Hermann structure with two chelates (hydrated and dehydrated, Figure 2.2); it resembles what a chelate might look like partway through the exchange process.
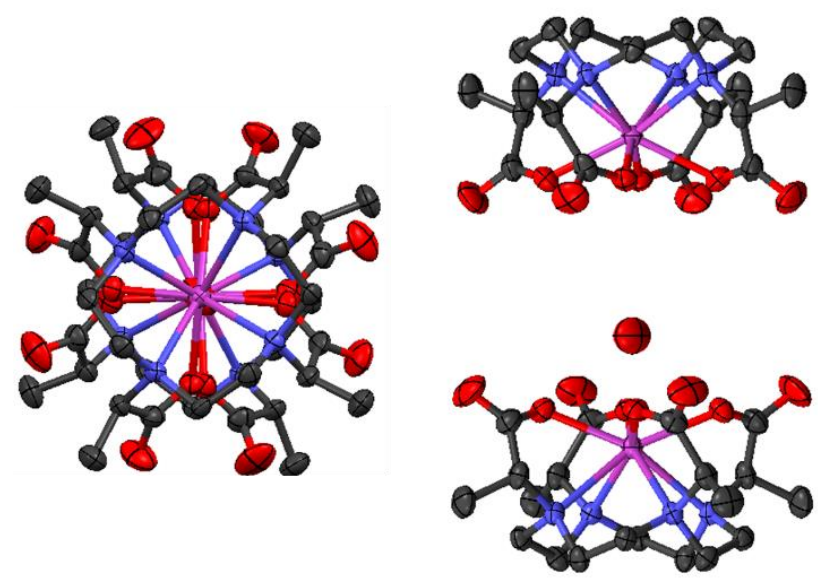

Figure 2.27: HoDOTMA crystal structure. Each hydrated and dehydrated pair is aligned along the axial ligand axis. For clarity the disordered water molecules, sodium, chloride, and hydrogen atoms are not shown.

The dehydrated chelate in the HoDOTMA unit cell is indisputably $q=0$, as no water in the crystal lattice lies within $3 \AA$ of the apical coordination site. The $d / c$ ratio for this chelate is small: 0.59 , consistent with a dehydrated chelate. The second chelate has a larger $\mathrm{d} / \mathrm{c}$ ratio: 0.64 , which is close to the boundary condition for hydration established in Figure 2.5. This second chelate has a water molecule apically located at $2.662 \AA$ from 
the metal ion. This distance represents an extraordinarily long Ho-O bond, raising the question of whether the water is truly bound to the metal, yet there is still clearly an interaction with the $\mathrm{Ho}^{3+}$ ion.

The crystal conformational changes associated with water exchange can be seen both in the suggested motion of the metal ion in Lukeš and Hermann's crystal and by our group's work with rigid chelates in solution. ${ }^{82,95}$ The TSAP chelates in the Lukeš and Hermann structure are distinct both in their hydration and in conformational parameters associated with hydration (Figure 2.2 and Table 2.1): the position of the metal ion in the coordination cage, the size of the $\mathrm{O}_{4}$ coordination square, etc. However, these two TSAP chelates are not indicative of exchange. The HoDOTMA structure has conformational parameters which lie between those hydrated and dehydrated TSAP chelates. Therefore these chelates may be indicative of the exchange process. The status of hydration in the HoDOTMA crystal supports the assertion that a TSAP' species that is not in exchange does not exist. It is the conformational differences in the chelates of the HoDOTMA unit cell that make it an interesting system for investigating solution state conformational changes associated with water exchange.

In the variable temperature ${ }^{1} \mathrm{H}$ NMR spectra of the conformationally rigid EuNB-DOTMA, the shift of the ligand proton resonances reflect the "breathing" motion of the chelate in the time-average dipolar shifts - the rate of shift change in the arm protons is faster than the ring protons. ${ }^{95}$ It is not possible to discern this fine motion in non-rigid systems, such as LnDOTMA, as the difference in shift of the SAP and TSAP isomers in equilibrium is large (Figure 2.28). However, the "breathing" motion of the 
chelate could influence the relative energies of the TSAP and SAP species and be reflected in their distribution in solution. Relative populations of the TSAP and SAP isomers are dependent on thermodynamics and the relative energy of the chelate conformations. According to thermodynamics the lower energy conformation is predominant at lower temperatures. Assuming the relative energies of TSAP and SAP isomers remain the same, increases in temperature should result in increased populations of the higher energy conformation. The most shifted axial protons of these chelates can be used to determine the distribution of coordination isomers (Figure 2.28).
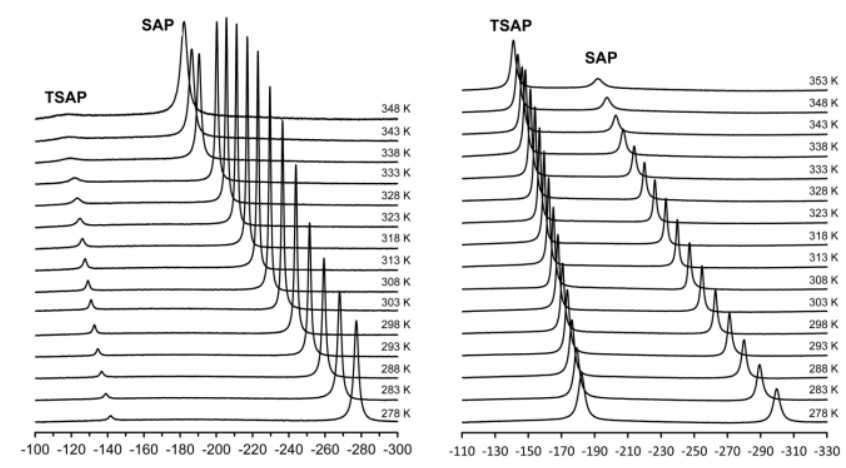

Figure 2.28: Temperature dependence of ${ }^{1} \mathrm{H}$ NMR shifts. The most shifted axial protons of HoDOTA (left) and HoDOTMA (right) are shown.

Previously unpublished variable temperature ${ }^{1} \mathrm{H}$ NMR experiments, conducted by our collaborators Profs. Silvio Aime and Mauro Botta, revealed some unusual behavior for the analogous chelate HoDOTA. For HoDOTA the SAP geometry is the predominate species in solution (64\% TSAP geometry at $298 \mathrm{~K}$ ), indicating that it has lower energy than the TSAP geometry. When the solution is heated, adding energy to the system, the distribution of isomers changes as expected, increasing in the population of the TSAP isomer. However, it can be seen in Figure 2.29 that this increase is only observed to about 
$330 \mathrm{~K}$; above this temperature the proportion of TSAP isomer begins to decrease again. Given that there was believed to be an association between the amount of TSAP isomer and its hydration state for later $\mathrm{Ln}^{3+}$ ion we thought that a similar study on HoDOTMA might afford insight into the solution state behavior of these chelates.

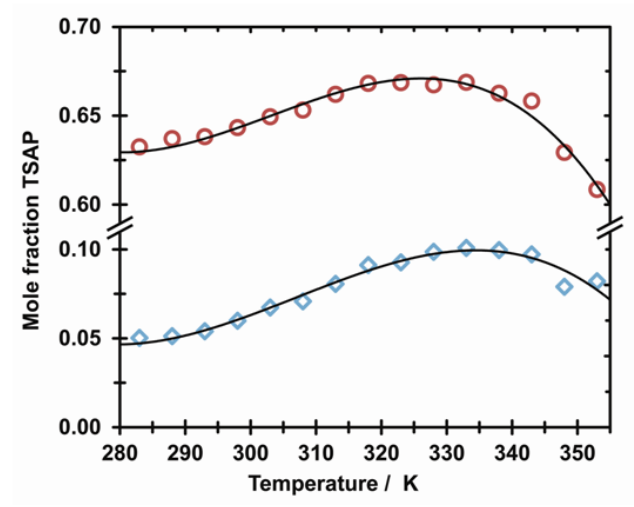

Figure 2.29: The relationship of the TSAP isomer population with temperature for solutions of HoDOTMA (circles) and HoDOTA (diamonds), as determined from ${ }^{1} \mathrm{H}$ NMR data.

For HoDOTMA the TSAP geometry is the predominant species in solution, indicating that it has lower energy than the SAP geometry. Therefore, it is expected that with increasing temperature more SAP conformation will be observed by ${ }^{1} \mathrm{H}$ NMR. However, as the temperature is increased the distribution of isomers does not move to increase the population of the SAP geometry, but instead we unexpectedly find an increase in the TSAP isomer (Figure 2.29). It is only at high temperatures (above $330 \mathrm{~K}$ ) that the system shows a reversal in this trend, increasing population of the higher energy SAP species.

This can be explained by a change to the time-averaged conformation of the ligand structure with increased water exchange kinetics and, therefore, the hydration state of the 
chelate. With increased water exchange rates the hydration state of the chelate decreases..$^{93,96,97}$ As a result of these conformational changes, the TSAP species for the LnDOTMA must be lowering its energy, allowing for the increase in its population in solution with increasing temperature. However, at some point the water molecule moves far enough away that changes in the ligand structure become negligible. These conformational changes associated with increasing rates of water exchange are also occurring in the SAP isomer and in the LnDOTA system. In the LnDOTMA chelate solution, when the decreased energy of the TSAP isomer with increased water exchange reaches its maximum, the expected increase in population of the SAP isomer is seen. In the LnDOTA chelate solution, the SAP and TSAP isomers both have an energy decrease associated with increased water exchange; when the decrease to the energy of the TSAP species reaches its maximum, the SAP isomer begins to re-populate.

To develop a more complete picture of the behavior of these chelates a complete relaxometric analysis $\left({ }^{17} \mathrm{O}\right.$ NMR and ${ }^{1} \mathrm{H}$ NMRD) was performed by Prof. Botta at the University of Eastern Piedmont in Alessandria, Italy. The results are shown in Figure 2.30. The dominant species in solution for HoDOTA is SAP and for HoDOTMA is TSAP. There are a few differences between these two species, but the most important is the fact that SAP has water exchange kinetics that are $50-100$ times slower than the TSAP. HoTETA, as a $q=0$ chelate, was included in this study as a control for outer sphere influence on relaxivity. To better understand the water exchange kinetics of these chelates the ${ }^{17} \mathrm{O}$ NMR transverse relaxation data was collected. ${ }^{17} \mathrm{O} \mathrm{R}_{2 \mathrm{p}}$ directly measures the water exchange rates of the chelate, allowing the influence of hydration to be probed 
more directly. From these data it is clear that the $\mathrm{Ho}^{3+}$ chelates probed have water exchange rates that differ dramatically (Figure 2.30 ). However, ${ }^{17} \mathrm{O}$ water does not distinguish between very fast exchange - which looks like the end of a bell curve - and outer sphere contribution. To differentiate these two scenarios, one must consider the shape of the NMRD profile.
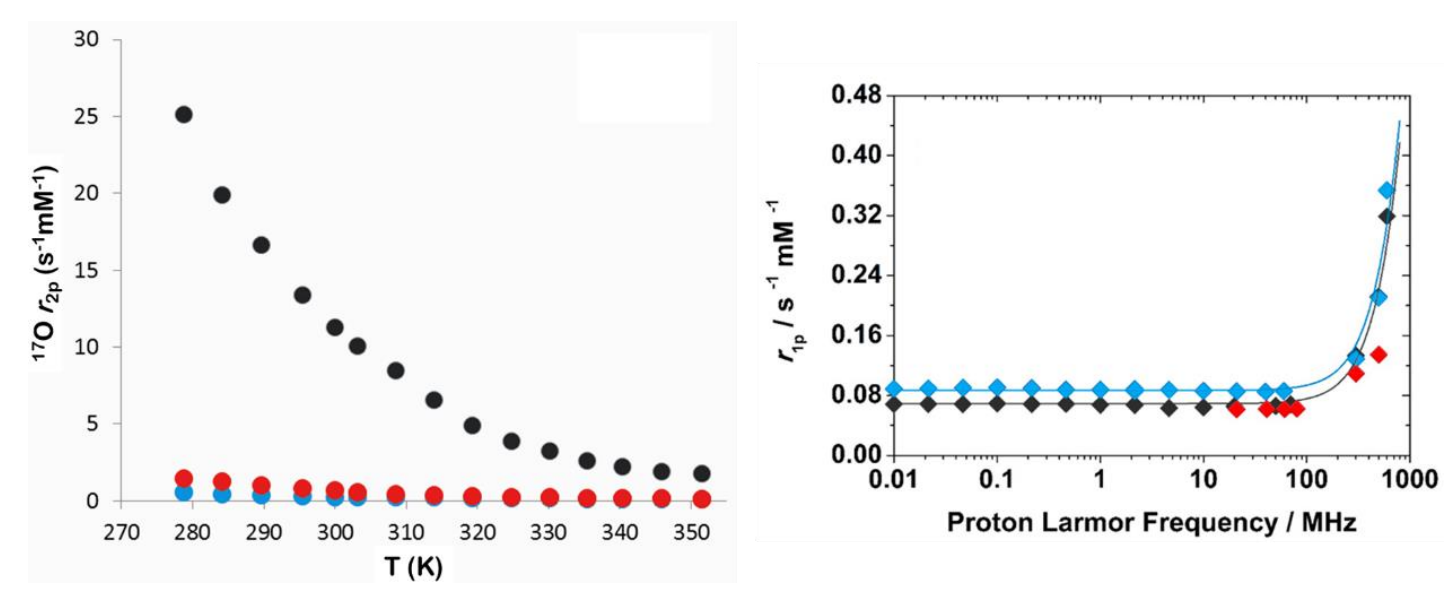

Figure 2.30: Variable temperature transverse relaxation ${ }^{17} \mathrm{O}$ at $11.7 \mathrm{~T}$ (left) and ${ }^{1} \mathrm{H}$ NMRD at $298 \mathrm{~K}$ (right) for HoDOTMA (blue), HoDOTA (black), and HoTETA (red) at $\mathrm{pH}=7.6$.

There are many parameters that influence the shape of NMRD profiles: tumbling in solution, electronic relaxation, and water exchange rates. HoDOTMA, HoTETA, and HoDOTA are similar in size and have similar $\tau_{R}$ values. Because these chelates are quickly tumbling, both HoDOTMA and HoDOTA are considered to be in the fast exchange regime - these faster water exchange rates are confirmed by the ${ }^{17} \mathrm{O}$ NMR data (Figure 2.30). At high fields where contributions from inner sphere dominate, HoDOTMA and HoDOTA have similar relaxivities, whereas HoTETA has lower 
relaxivity as expected for a chelate without inner sphere water (Figure 2.30). At low fields, where outer sphere effects dominate, the HoTETA and HoDOTA have similar relaxivities, and HoDOTMA has a slightly increased relaxivity. This difference between HoDOTA and HoDOTMA at low fields is attributed to the slightly different electronic relaxation time constants of these two chelates, not relative hydration.

The NMRD profiles of $\mathrm{Ho}^{3+}$ chelates have very different characteristics compared to the more common profiles of $\mathrm{Gd}^{3+}$ chelates, and fitting these profiles to SBM theory is extremely complex. There are few published examples of ${ }^{1} \mathrm{H}$ NMRD profiles for non-Gd ${ }^{3+}$ chelates; those that have been fitted use ambiguous parameters set by Merbach and co-workers. ${ }^{98-100}$ Because there is much to be gained from qualitatively assessing the data, and relatively little that would be further gained from quantitatively fitting the data, we have chosen not to pursue fitting the NMRD profiles of the $\mathrm{Ho}^{3+}$ chelates.

\subsection{Conclusions}

The LnDOTMA crystal structure data supports the idea that the $\mathrm{Ln}^{3+}$ ion moves within the coordination cage with water exchange. ${ }^{82}$ These crystal data provide a picture of water exchange: early in the LnDOTMA series the lanthanide-water distance slightly decreases, then increases late in the $\mathrm{Ln}^{3+}$ series until the water is no longer associated with the metal as the effective coordination number decreases. Along with lanthanidewater distances, we analyzed many other parameters of the chelate structures, such as the presence of apical water, twist angles of the chelate, position of the lanthanide in its coordination cage, and size of the $\mathrm{O}_{4}$ coordination square. These interrelated variables all indicate that there is no clear delineation between hydrated and dehydrated, as currently 
considered, but instead a spectrum of ligand conformations that gradually occur as the chelates become more dehydrated. LnDOTMA is the first full set of lanthanide chelates to exclusively crystallize in TSAP geometry to allow for these observations.

The conformational changes associated with decreased coordination number in the LnDOTMA crystals provide structural information about the chelate undergoing water exchange. This has potential influence on the way that hydration is thought of in LnDOTA-type chelates. The conformational differences seen in the solid state reflects the solution state. This is supported by the solution studies of HoDOTMA, where we observe the increase in population of the major isomer with increased temperature in variable temperature ${ }^{1} \mathrm{H}$ NMR studies of HoDOTMA. This decrease in energy of the lowest energy isomer is possible with conformational changes to the chelate, which are associated with lengthening metal-water distances.

In order to achieve the goal of a more effective contrast agent, a common focus of ligand design is to produce optimal water exchange kinetics and tumbling rates for lanthanide chelates. However, previous research efforts have commonly over-looked the influence of water position and its relationship with water exchange kinetics. The indication that conformational motion occurs with water exchange in the solution state demands that the relationship of hydration $\left(q / r^{6}\right)$ and water exchange kinetics be considered. Without this consideration, experimental data fitted to the SBM equations cannot be interpreted accurately, hindering the process of designing optimized contrast agents. Future studies to further elucidate the relationship between apical water position and water exchange kinetics are necessary to be able to predict the optimal water exchange rates. However, 
from these data it seems that optimal water exchange kinetics will be slower than previously predicted. 


\section{CHAPTER 3: INVESTIGATIONS INTO THE RELAXOMETRIC PROPERTIES OF VARIOUS ISOMERS OF GdNB-DOTMA}

\subsection{Background}

The rigidity of lanthanide NB-DOTMA chelates results in low metal dissociation constants and makes this a ligand of interest for research. Four isomeric chelates are possible as a result of the macrocycle and pendant arm substitutions to the DOTA framework: the corner and side regioisomers of the TSAP $(S-\Delta(\delta \delta \delta \delta))$ and the SAP $(S-\Lambda(\delta \delta \delta \delta))$ chelates (Figure 3.1). ${ }^{67-70}$ A full relaxometric analysis of the four possible

isomers of GdNB-DOTMA has yet to be completed. ${ }^{64,67,68}$ The completion of such a study should provide insights into the parameters controlling the effectiveness of these chelates as contrast agents. The previously determined orientation of the benzylic group ${ }^{67}$ which differs between the corner and the side isomer, may influence the parameters controlling relaxivity. This could be caused either by affecting the hydrodynamic shape and size of the chelate and altering the rate at which the isomers tumble in solution or by causing different distortions to the coordination cage and impacting the water exchange rates or electronic relaxation. The rigidity of the LnNBDOTMA chelates enables unobscured probing of the physicochemical properties specifically, the fine details of structure/activity relationships between the coordination chemistry of the $\mathrm{Gd}^{3+}$ chelates and water exchange kinetics. 

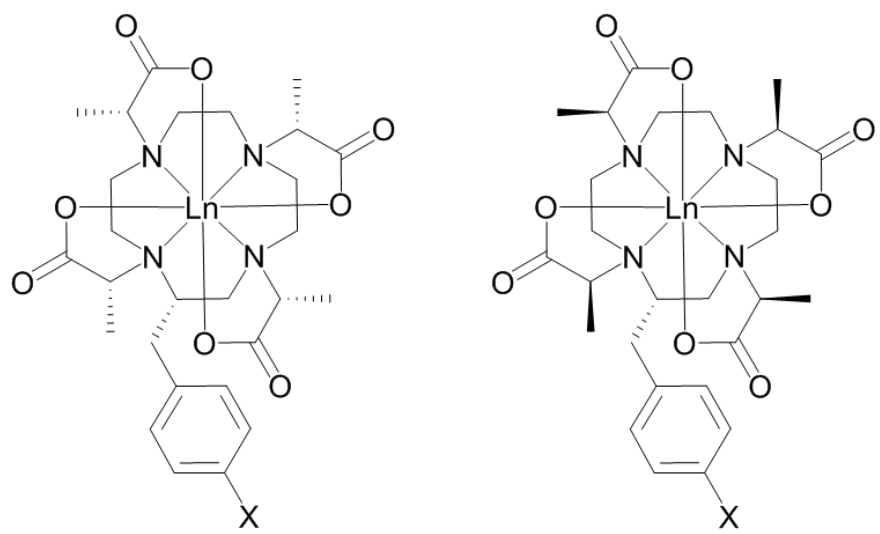

$$
\begin{array}{ll}
\text { LnNB-DOTMA } & \mathrm{X}=-\mathrm{NO}_{2} \\
\text { LnIB-DOTMA } & \mathrm{X}=-\mathrm{NCS}
\end{array}
$$

Figure 3.1: Connectivity of the S-SSSS- (left) and S-RRRR- (right) chelates that generate TSAP and SAP geometry, respectively.

It is well established that the kinetics of water exchange are $50-100$ times faster in the TSAP isomer than in the SAP isomer. ${ }^{70,74,101-103}$ Current contrast agents have suboptimal water exchange rates. The established dogma in the field indicates that a slowly tumbling chelate with faster water exchange will have higher relaxivity than one with slower water exchange. However, it is possible for a chelate to have water exchange kinetics that are too fast. Recently, we have shown that water exchange is intrinsically linked to the hydration state $\left(q / r^{6}\right)$ of the chelate. For very rapidly exchanging chelates this can have a negative impact on relaxivity: as hydration decreases, so will the effectiveness of the chelate. Dogma predicts that the TSAP isomer, which has a more rapid water exchange relative to the SAP isomer, will have higher relaxivity in a slowly tumbling system. However, in practice we find that the opposite is true. This observation is attributed to the reduced hydration state associated with very rapid exchange, resulting 
in a lower relaxivity. ${ }^{64}$ To better understand the relaxometric properties of rapidly exchanging $\mathrm{Gd}^{3+}$, studies of chelates with single well-defined coordination chemistry, such as the isomers of GdNB-DOTMA, are invaluable. ${ }^{17} \mathrm{O}$ NMR can be used to accurately determine the water exchange dynamics of these $\mathrm{Gd}^{3+}$ chelates.

\subsection{Determination of water exchange kinetics in the isomers of GdNB-DOTMA}

The temperature dependence of the ${ }^{17} \mathrm{O}$ transverse relaxation rate constant for solutions of $\mathrm{Gd}^{3+}$ chelates can be used to determine the water exchange kinetics of the chelate. However, the concentration demands of ${ }^{17} \mathrm{O} \quad R_{2 \mathrm{p}}$ relaxometric experiments are comparatively high - higher than those of ${ }^{1} \mathrm{H} R_{1 \mathrm{p}}$ studies. In unpublished work, Ben Webber had previously prepared and isolated each of the four possible isomers of GdNB-DOTMA. These samples were sufficient to obtain ${ }^{1} \mathrm{H}$ NMRD profiles, as well as preliminary ${ }^{17} \mathrm{O} R_{2 \mathrm{p}}$ profiles, for each isomer. However, confidence in the ${ }^{17} \mathrm{O} R_{2 \mathrm{p}}$ profiles recorded was not high due to the sample limitations.

To complete a robust ${ }^{17} \mathrm{O}$ transverse relaxation analysis, greater quantities of each isomer were prepared: the $S$-SSSS- and $S$-RRRR-GdNB-DOTMA stereoisomers were remade, and the corner and side isomers were separated by preparative RP-HPLC using the same methods employed previously by Ben Webber. Samples were analyzed in the laboratory of Prof. Botta at the University of Eastern Piedmont in Alessandria, Italy. 


\section{CHAPTER 3}
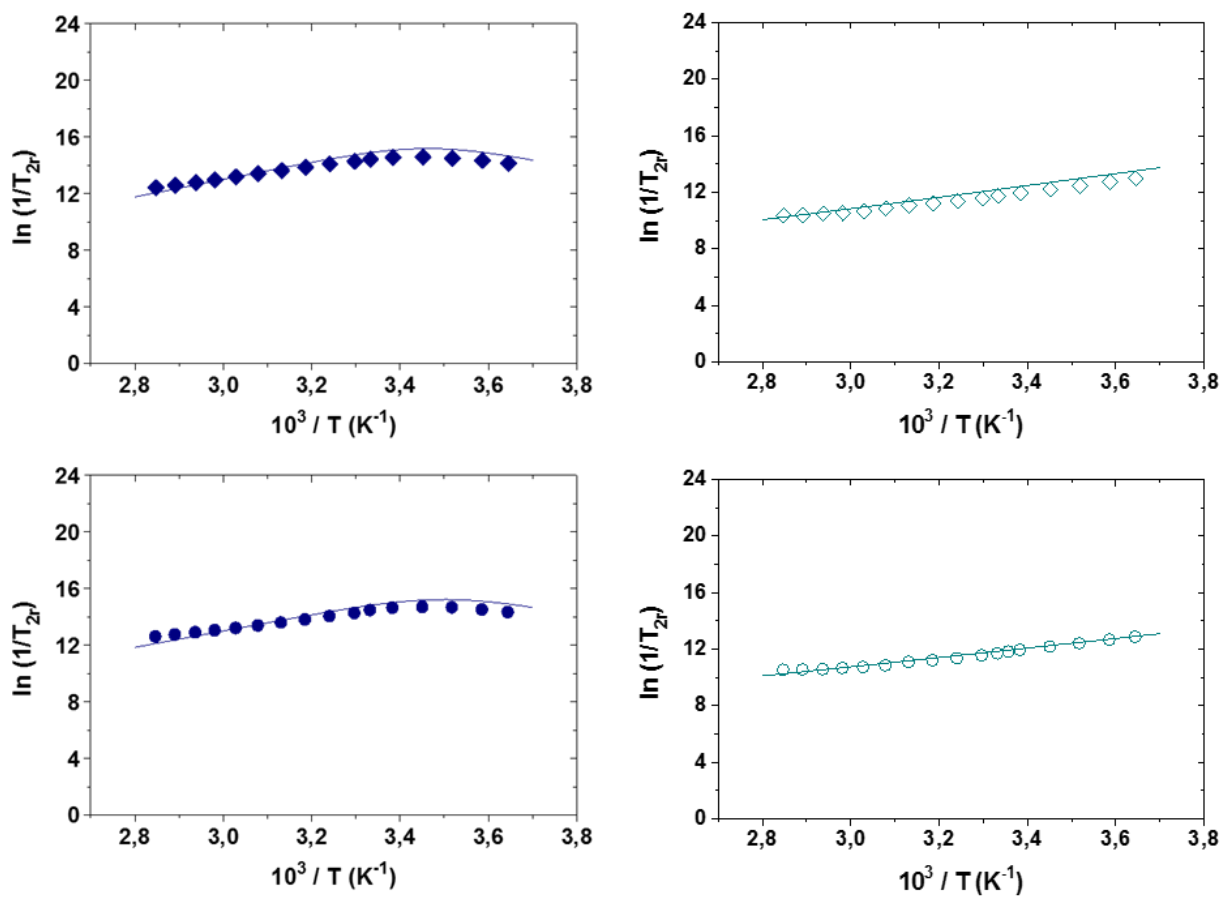

Figure 3.2: Variable temperature ${ }^{17} \mathrm{O}$ transverse relaxation data for the corner (top) and side (bottom) isomers of S-RRRR-GdNB-DOTMA (left) and S-SSSS-GdNB-DOTMA (right) chelates (11.74 T).

The transverse relaxation rate constants of the solvent water $\left({ }^{17} \mathrm{O} R_{2 \mathrm{p}}\right)$ were determined as a function of temperature at $11.74 \mathrm{~T}$. These data are presented in Figure 3.2 as the natural $\log$ of ${ }^{17} \mathrm{O} R_{2 \mathrm{p}}$ against $1000 /$ temperature in Kelvin. Both SAP isomers (S-RRRR- corner and side) exhibit ${ }^{17} \mathrm{O}$ relaxometric temperature profiles that reach maximum values in the temperature range studied (Figure 3.2, left). No maxima are observed in the ${ }^{17} \mathrm{O}$ relaxometric temperature profiles of the TSAP isomers ( $S$-SSSS- corner and side) (Figure 3.2, right). The lower the temperature to which the profile maximum corresponds the faster the water exchange. These profiles indicate that, as expected, the water exchange rates of both the SAP isomers are slower than those of the TSAP isomers. 
Values for $\tau_{M}$ are afforded by fitting these profiles to theory (Table 3.1). ${ }^{104-106}$ These data show that the side isomer has somewhat faster water exchange kinetics than the corner isomer in both SAP and TSAP coordination isomers. Water exchange kinetics are influenced by electrostatic forces at the binding site. The presence of the benzylic substituent causes distortions to the ligand cage; the relative positions of this substituent are not the same for the corner and side regioisomers. The somewhat faster exchange kinetics of the side isomers indicate that these distortions must have some effect on the positions of the donor atoms in the coordination cage - slightly altering the electrostatic forces at the water exchange site - to cause the water exchange kinetics to be different between the regioisomers.

Table 3.1: Fitting parameters for the ${ }^{17} \mathrm{O}$ transverse relaxation data of GdNB-DOTMA. For all complexes $a=4.0 \AA, D^{298}=2.24 \times 10^{-5} \mathrm{~cm}^{2} \mathrm{~s}^{-1}, D^{310}=3.10 \times 10^{-5} \mathrm{~cm}^{2} \mathrm{~s}^{-1}$, $E V=1.0 \mathrm{~kJ} \mathrm{~mol}^{-1}$, and $E_{R}=16.0 \mathrm{~kJ} \mathrm{~mol}^{-1}$.

\begin{tabular}{ccccc}
\hline & \multicolumn{2}{c}{$S$-RRRR- } & \multicolumn{2}{c}{$S$-SSSS- } \\
& Corner & Side & Corner & Side \\
\hline$A / \hbar\left(10^{6} \mathrm{rad} \mathrm{s}^{-1}\right)$ & -3.6 & -3.7 & -3.0 & -2.9 \\
$\tau_{\mathrm{M}(\mathrm{ns})}$ & 60 & 49 & 4 & 3 \\
$\Delta H\left(k \mathrm{~J} \mathrm{~mol}^{-1}\right)$ & 50.2 & 46.8 & 34.9 & 27.4 \\
\hline
\end{tabular}

Hydration and water exchange are parameters that directly influence the efficacy of contrast agents. The water exchange kinetics determined by ${ }^{17} \mathrm{O}$ NMR data were used to 
improve the analysis of the GdNB-DOTMA relaxometric properties and reliably calculate parameters influencing effectiveness of these chelates.

\section{3 ${ }^{1} \mathrm{H}$ Relaxometric studies of the isomers of the GdNB-DOTMA}

The ${ }^{1} \mathrm{H}$ NMRD profiles of the GdNB-DOTMA chelates show profiles typical of low molecular weight chelates; at low fields there is steady and high relaxivity until it disperses above $1 \mathrm{MHz}$ to decrease with increasing field. Both the corner and side isomers of $S$-RRRR-GdNB-DOTMA (SAP) have higher relaxivity than either the corner or side isomer of S-SSSS-GdNB-DOTMA (TSAP) (Figure 3.3). For the SAP isomers the side regioisomer has higher relaxivity than the corner, and for the TSAP isomers the corner regioisomer has higher relaxivity than the side. These profiles were collected at 25 and $37{ }^{\circ} \mathrm{C}$; the lower temperature results in a higher relaxivity across all fields for all of the LnNB-DOTMA chelates. 

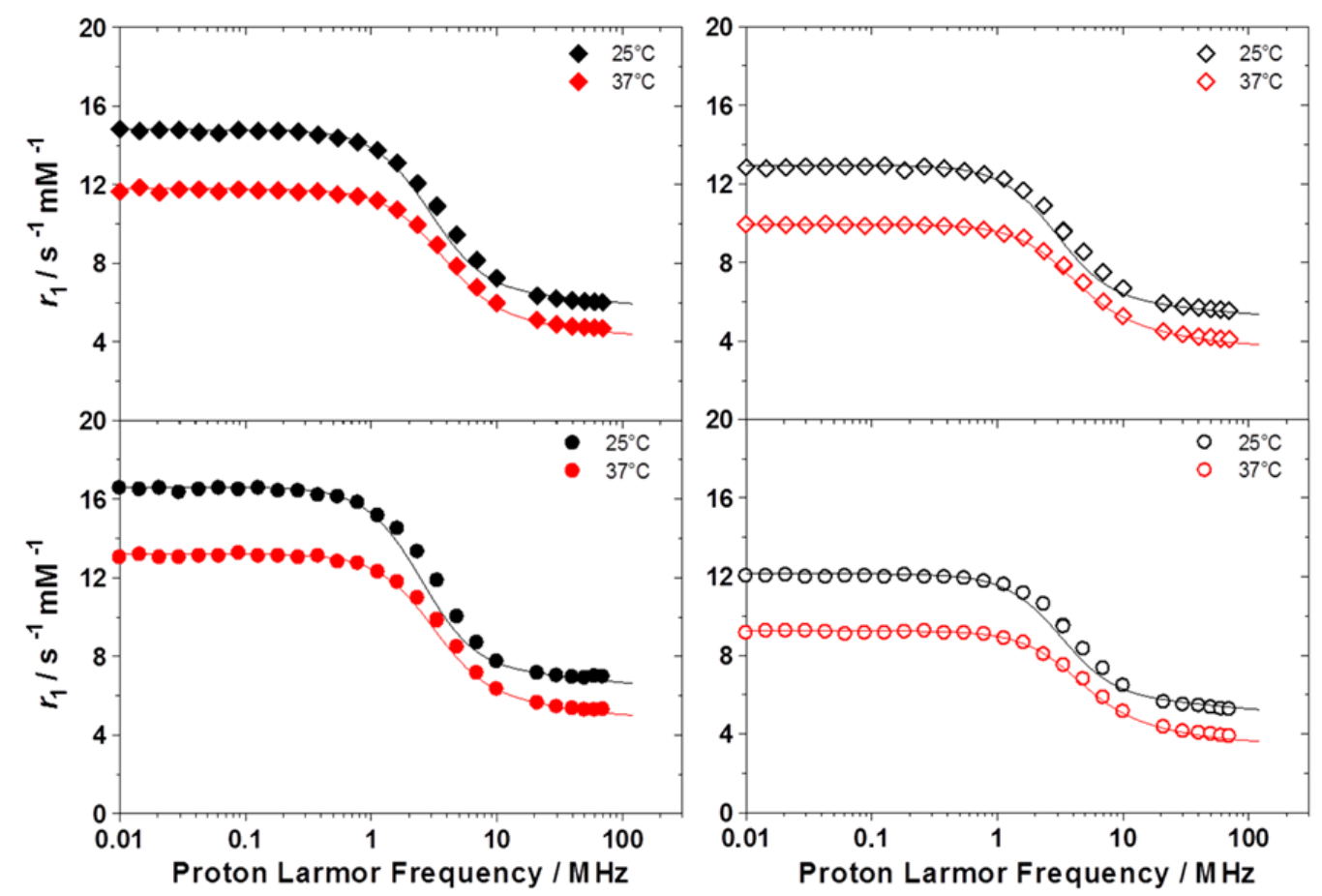

Figure 3.3: ${ }^{1} \mathrm{H}$ NMRD profiles of SAP (left) and TSAP (right) for corner (top) and side (bottom) isomers of GdNB-DOTMA. Fittings shown include ${ }^{17} \mathrm{O}$ NMR data.

The ${ }^{1} \mathrm{H}$ NMRD profiles can be fit to the SBM equations to determine the influence of various parameters from SBM theory on relaxivity of the chelate. Care must be taken when performing calculations to fit the SBM equations to an NMRD profile. Certain parameters have co-dependent relationships with others in the SBM equations, i.e. changing certain parameters (some of which are fixed during fitting) can cause another to change. This means that more than one set of parameters can be used to fit the data well. Judgment must be exercised in determining what fitting parameters are reasonable. Using empirically determined values for the fitting of the profiles improves the accuracy of this process. 
Table 3.2: Relaxometric parameters obtained from the fit of the ${ }^{1} \mathrm{H}$ NMRD profiles of $S$-SSSS- and S-RRRR-GdNB-DOTMA. For all complexes $q=1, a=4.0 \AA$,

\begin{tabular}{|c|c|c|c|c|c|}
\hline \multirow{2}{*}{ Complexes } & $r_{1}^{[\mathrm{a}]}$ & $r^{[\mathrm{d}]}$ & $\Delta^{2}$ & $\tau_{\mathrm{V}}$ & $\tau_{\mathrm{R}}$ \\
\hline & $\left(\mathrm{mM}^{-1} \mathrm{~s}^{-1}\right)$ & $(\AA)$ & $\left(10^{19} \mathrm{~s}^{-2}\right)$ & (ps) & (ps) \\
\hline SAP corner & 6.3 & 3.0 & 1.5 & 17 & 112 \\
\hline SAP side & 7.1 & 3.0 & 1.2 & 18 & 134 \\
\hline TSAP corner & 5.9 & 3.1 & 1.6 & 19 & 118 \\
\hline TSAP side ${ }^{[b]}$ & 5.6 & 3.1 & 1.8 & 20 & 115 \\
\hline TSAP side ${ }^{[c]}$ & 5.6 & 3.17 & 1.78 & 21 & 134 \\
\hline
\end{tabular}

[a] recorded at $20 \mathrm{MHz}$ and $298 \mathrm{~K}$; [b] first fit; [c] second fit; [d] fixed during fitting, along with $\tau_{\mathrm{M}}$ from Table 3.1 .

The ${ }^{1} \mathrm{H}$ NMRD profiles of the four GdNB-DOTMA isomers were initially fit using the $\tau_{\mathrm{M}}$ values determined by ${ }^{17} \mathrm{O}$ NMR relaxometry and fixing the value of $r_{\mathrm{GdH}}$ to arbitrary values previously employed in the fitting of NMRD profiles of chelates with fast and very fast exchange: $r_{\mathrm{GdH}}=3.0 \mathrm{~A}$ for the SAP isomers and $r_{\mathrm{GdH}}=3.1 \mathrm{~A}$ for the TSAP isomers. $^{28,48}$ These values are not intended to reflect actual changes in bond length, but instead to adjust the hydration parameter. This approach of using different values of $r_{\mathrm{GdH}}$ affords a method by which hydration $\left(q / r^{6}\right)$ can be varied without changing the format of the SBM equations usually employed in this fitting. This means that we employ a hydration number of $q=1$ and all differences in hydration are accounted for by variation in the $r_{\mathrm{GdH}}$ distance 
$r_{\mathrm{LnH}}$ distances are near impossible to measure directly. To approximate the average $r_{\mathrm{LnH}}$ distance in solution there are many techniques used: drawing estimates based on X-ray diffraction, ${ }^{17,107}$ fitting of $\mathrm{Gd}^{3+}$ enhanced proton relaxation rate, ${ }^{51,108}$ electron spin-echo envelope modulation spectroscopy, ${ }^{109}$ and continuous wave ${ }^{110}$ or systematic pulsed ${ }^{111}$ electron-nuclear double resonance. The value selected for $r_{\mathrm{GdH}}$ typically is in the range of $2.90-3.13 \AA$. The use of different $r_{\mathrm{GdH}}$ values for the SAP and TSAP isomers were justified both by the measured differences in hydration of the $\mathrm{Eu}^{3+}$ chelates in solution ${ }^{69}$, 70 and the differences in $\mathrm{Gd}^{3+}-\mathrm{OH}_{2}$ distances in crystal structures of $\operatorname{GdDOTMA}^{63}$ (a TSAP isomer) and GdTCE-DOTA ${ }^{74}$ (a SAP isomer).

Fitting the NMRD profiles on the basis of these $r_{\mathrm{GdH}}$ values afforded $\tau_{\mathrm{R}}$ values that were more or less consistent between three of the four GdNB-DOTMA isomers (Table 3.2). This result was largely to be expected for isomeric chelates which would intuitively be expected to be of similar hydrodynamic volume and therefore tumble at the same rate. It has previously been shown that the corner SAP and corner TSAP isomers of TbNB-DOTMA tumble at comparable rates. ${ }^{31}$ However, the side SAP isomer of GdNB-DOTMA was found to have both a markedly longer $\tau_{R}$ value and higher relaxivity. This raises the interesting prospect that the different regiochemistry of the benzylic substituent gives rise to different rates of tumbling in solution.

There is no intrinsic chemical relationship between the values of $\tau_{\mathrm{R}}$ and $r_{\mathrm{GdH}}$ for chelates in solution. However, in the SBM model these two parameters have a mutually compensating relationship with one another. Thus the value of $\tau_{\mathrm{R}}$ obtained from fitting is dependent on the value of $r_{\mathrm{GdH}}$ used in the fitting. ${ }^{51}$ With this in mind we more closely 
inspected the empirically determined water exchange rates that directly impact the chelate hydration in solution. $\tau_{\mathrm{M}}$ is commonly considered equal to the inverse of the water exchange rate, but this is an approximation that only works well when water exchange is merely fast. When water exchange is very fast the time the water spends both associated with the chelate $\left(\tau_{\mathrm{M}}\right)$ and off the chelate ( $\left.\tau_{\text {off }}\right)$ become relevant (Eq. 3.1).

$$
\frac{1}{k_{e x}}=\tau_{M}+\tau_{o f f}
$$

\section{Equation 3.1: Defining the rate of water exchange $\left(k_{e x}\right)$}

The value of $\tau_{\text {off }}$ on average must be a short time that is of negligible duration when the water residence lifetime is long. However, we hypothesize that with very fast water exchange the water residence lifetime ( $\tau_{\mathrm{M}}$ ) begins to approach $\tau_{\text {off }}$, making $\tau_{\text {off }}$ no longer negligible to the discussion of water kinetics and hydration (Figure 3.4). There is no way to directly measure $\tau_{\text {off }}$ or $r_{\mathrm{GdH}}$, but for illustrative purposes we have examined the effect of $\tau_{\text {off }}=50 \mathrm{ps}$ (a negligible period of time compared to a $\tau_{\mathrm{M}}$ value of nano- to microseconds) with exchange occurring over a distance of $r_{\mathrm{GdO}}$ between 2.4 and $4.0 \AA$. With these chosen parameters there is a notable increase in the amount of time the chelate is without water during a given period of time across a range of relative $\tau_{\mathrm{M}}$ values (Figure 3.4, red line). The impact of this on the overall hydration of the chelate is significant at the faster $\tau_{\mathrm{M}}$ values (Figure 3.4, blue line). The differentiation between "fast" and "very fast" - the division when fast water exchange starts negatively impacting hydration - seems to lie between $\tau_{\mathrm{M}}=10$ and $20 \mathrm{~ns}$ in Figure 3.4, but will undoubtedly vary depending on the actual value of $\tau_{\text {off. }}$ 


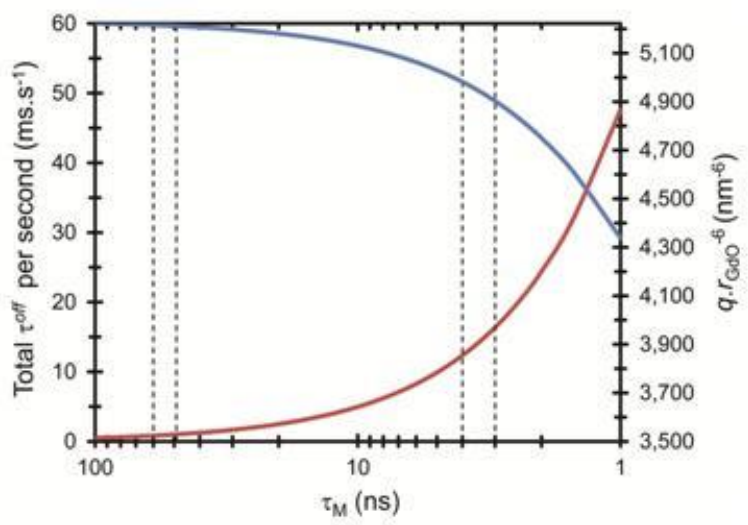

Figure 3.4: The decrease in hydration, resulting from $\tau_{\text {off }}$ becoming significant, as water exchange becomes very fast. The red curve indicates the amount of time the water spends off the chelate in a single time repetition of 1 second. The blue curve indicates the decrease in hydration as a result of $\tau_{\text {off }}$ becoming significant. The dotted lines are located at the $\tau_{\mathrm{M}}$ values of the four LnNB-DOTMA chelates.

When water exchange is very fast the chelate will spend more time in the dehydrated state. Additionally, as water exchange becomes increasingly fast the time that the chelate spends dehydrated becomes increasingly significant. The two SAP isomers of GdNB-DOTMA are in the fast water exchange regime; however, the order of magnitude faster water exchange rates of the two TSAP isomers should be considered "very fast." This very fast water exchange results in diminished hydration of the TSAP isomer, as a result of the contribution of $\tau_{\text {off }}$ being more significant. This analysis shows that the value of $\tau_{\mathrm{M}}$ can affect the appropriate value of $q / r^{6}$.

The very fast water exchange of the TSAP isomers demands that a longer $r_{\mathrm{GdH}}$ distance be used in fitting than is employed for the SAP isomers - this is accommodated in the initial fitting by using $r_{\mathrm{GdH}}=3.0$ and $3.1 \AA$ for SAP and TSAP chelates, respectively. In 
the initial fits of the ${ }^{1} \mathrm{H}$ NMRD profiles this resulted in $\tau_{R}=115$ and $118 \mathrm{ps}$ for the side and corner TSAP isomers, respectively and $\tau_{\mathrm{R}}=134$ and $112 \mathrm{ps}$ for the side and corner SAP isomers, respectively. The slower tumbling seen in the side SAP isomer was unexpected based on the previous TbNB-DOTMA study; however, because the relative orientations of the benzylic substituent are different for the corner and side isomers, there is the possibility that the two regioisomers could have different tumbling in solution.

From Figure 3.4 it can be seen that changing $\tau_{\mathrm{M}}$ from 4 to 3 ns (a $25 \%$ change in the TSAP regioisomers) could have a profound effect on $q / r^{6}$. Although the difference in $\tau_{M}$ appears small (just $1 \mathrm{~ns}$ ), this difference should lead to a significant difference in the hydration states of the two chelates. In contrast the difference between the $\tau_{M}$ values for the two regioisomers of the SAP geometry has no appreciable effect on $q / r^{6}$.

Given that the value of $r_{\mathrm{GdH}}$ will influence the value of $\tau_{\mathrm{R}}$ obtained from the fitting, we reevaluated the fitting for the ${ }^{1} \mathrm{H}$ NMRD profile of the TSAP side isomer (Table 3.2). In this fitting we started with a fixed value of $\tau_{R}$ (the same as obtained for the SAP side isomer $134 \mathrm{ps)}$ and allowing $r_{\mathrm{GdH}}$ to vary. This afforded a value of $r_{\mathrm{GdH}}=3.17 \mathrm{~A}$. This $r_{\mathrm{GdH}}$ value seems reasonable based on Figure 3.2 and suggests that the corner and side regioisomers have different rates of tumbling, but that tumbling is broadly similar across all corner isomers and across all side isomer.

\subsection{Conclusions}

By remaking the rigid chelates we were able to obtain robust experimental ${ }^{17} \mathrm{O}$ data. The analysis of these data indicates that the water exchange in the GdNB-DOTMA chelates is 
somewhat faster for the side isomers than the corner isomers. These differences in water exchange kinetics between regioisomers have a particular impact on the TSAP isomer. The very fast water exchange rates result in a decrease in chelate hydration that is reflected in the reduced relaxivity of the TSAP chelates.

The experimentally determined $\tau_{\mathrm{M}}$ values allow for improved fits of the previously collected ${ }^{1} \mathrm{H}$ NMRD profiles. These new fits of the GdNB-DOTMA isomers indicate a potential difference in tumbling of the regioisomers; the side isomers have slower rotational correlation times than the corner isomers. This would seem to be the result of the different orientations of the benzylic substituent relative to the macrocycle giving rise to slightly different hydrodynamic volumes.

The analysis of GdNB-DOTMA chelates, both from this chapter and published work elsewhere, challenges current ideas on hydration in the field: faster is not always better. The ${ }^{1} \mathrm{H}$ NMRD profiles of the TSAP and SAP regioisomers shows that the relaxivities of the TSAP regioisomers are consistently lower than those of the SAP isomers at all fields, with a larger difference at low fields. The relaxivity of these chelates is limited by their tumbling in solution, but their relative relaxivities are influenced by water exchange kinetics impacting chelate hydration.

The different tumbling of the regioisomers of GdNB-DOTMA has potential influence on the efficacy of their derivatives as BFCs. Tumbling rates are in direct relation to the hydrodynamic radius of these molecules. The slower tumbling of the side isomer of GdNB-DOTMA has direct influence on the relaxivity of this agent, but also may indicate a relative position of the benzylic substituent that could allow for stronger interactions of 
the moiety with macromolecules of interest. Further studies are required to confirm the relative tumbling rates of the regioisomers.

As the nitro moiety on the benzylic substituent is readily modified to bind macromolecules and slow tumbling, GdNB-DOTMA has potential for application as a BFC. However, neither the SAP nor the TSAP isomers of GdNB-DOTMA have optimal water exchange kinetics. That the relaxivities of the slower exchanging SAP isomers were closer to optimal than the very fast exchanging TSAP isomers gives a starting point for experimentally determining the optimal $\tau_{M}$ value. A future study of chelates with a range of water exchange kinetics intermediate to those of the SAP and TSAP isomers of GdNB-DOTMA may empirically determine the optimal value for water residence lifetime. 


\section{CHAPTER 4: EFFECTS OF REGIOISOMERISM IN THE LnNB-DOTA SYSTEM}

\subsection{Background}

Bifunctional chelators (BFC) are popular for development of targeting agents: they provide both binding of the active metal and a targeting moiety for interacting with biological molecules of interest. Although NB-DOTA is not a BFC, it is a stable precursor to the bifunctional chelator IB-DOTA (Figure 4.1). The $\mathrm{H}_{4} \mathrm{NB}-\mathrm{DOTA}$ ligand is more amenable to study because it is commercially available and more stable than IB-DOTA. The properties of the ligand or its chelates are not expected to be appreciably altered by the different moiety on the benzyl group. $\mathrm{H}_{4}$ NB-DOTA was first synthesized and published by Meares and co-workers in $1988 .{ }^{112}$ It was developed as a potential BFC for radiotherapy applications, specifically to provide inert chelation of ${ }^{90} \mathrm{Y}^{3+}$. An investigation into the kinetic and thermodynamic stability of this ligand with $\mathrm{Ln}^{3+}$ ions was published in 2004 by Woods et al. ${ }^{72}$ This paper also examined some of the solution state behavior of these chelates and determined that the conformation of the macrocyclic ring was frozen: therefore, the interconversion of the two coordination isomers (SAP and TSAP) occurs through pendant arm rotation. ${ }^{72}$ Although the occurrence of regioisomers in $\mathrm{Ln}^{3+}$ chelates of macrocycle substituted DOTA derivatives had been previously published ${ }^{71}$ regioisomeric chelates were not identified or discussed in the 2004 study of LnNB-DOTA chelates. 


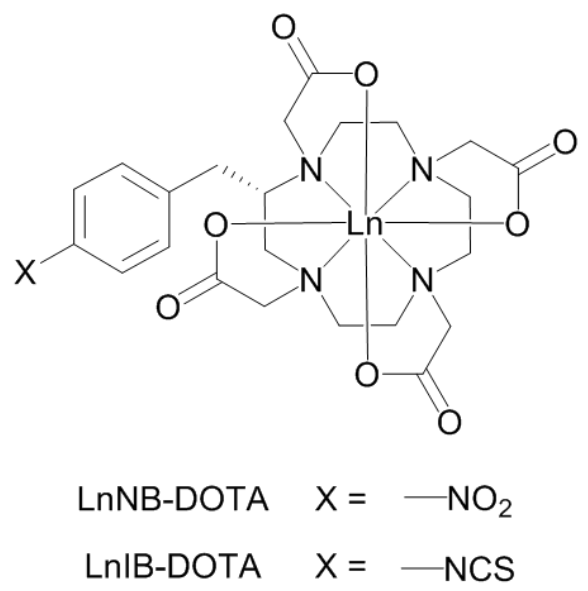

Figure 4.1: The connectivity structure of LnIB-DOTA and its more stable analog LnNB-DOTA

Differentiation of the regioisomers of the related LnNB-DOTMA chelates was found to occur when the $\mathrm{Ln}^{3+}$ ion was introduced into the ligand. ${ }^{68}$ This spurred the idea that similar regioisomers of the LnNB-DOTA chelates must also be possible. Typically BFCs are used by first reacting the BFC ligand with a biomolecule to produce a bioconjugate. The ligand contained within this bioconjugate is then used to chelate the preferred metal ion. This makes it extremely difficult to purify or separate the products of the crude chelation product, and leads to multiple regioisomeric products - with potential for differing chemical properties - within the bioconjugate. Regioisomerism in this system has potential to impact the effectiveness of NB-DOTA derivatives as BFCs.

4.2 Synthesis and purification of chelates: does regioisomerism occur in the LnNB-DOTA system?

The $\mathrm{Eu}^{3+}, \mathrm{Gd}^{3+}$, and $\mathrm{Yb}^{3+}$ chelates of NB-DOTA were prepared by addition of the corresponding lanthanide chloride to a solution of the ligand at $\mathrm{pH} 5.5$ in aqueous 
solutions at $40{ }^{\circ} \mathrm{C} .{ }^{1} \mathrm{H}$ NMR analysis of the crude reaction product of $\mathrm{Eu}^{3+}$ and YbNB-DOTA at $400 \mathrm{MHz}$ shows peaks consistent with four species in solution. The side axial protons are the most shifted resonances in the ${ }^{1} \mathrm{H}$ NMR spectrum of EuNB-DOTA $(10-40 \mathrm{ppm})$. By examining this region of the spectrum two primary species can readily be identified (Figure 4.2): one SAP isomer (resonances between 30 and $40 \mathrm{ppm}$ ) and one TSAP isomer (resonances between 10 and $20 \mathrm{ppm}$ ). These two species were also identified in the 2004 paper. However, closer inspection of Figure 4.2 shows that additional species, both SAP and TSAP, are also present in the crude reaction solution. The smaller peaks visible in the spectrum of the EuNB-DOTA crude reaction product are difficult to discern in the ${ }^{1} \mathrm{H}$ NMR of EuNB-DOTA recorded at $270 \mathrm{MHz}$ (published in 2004) due to overlap of the proton signals and the relative size of the minor species peaks.

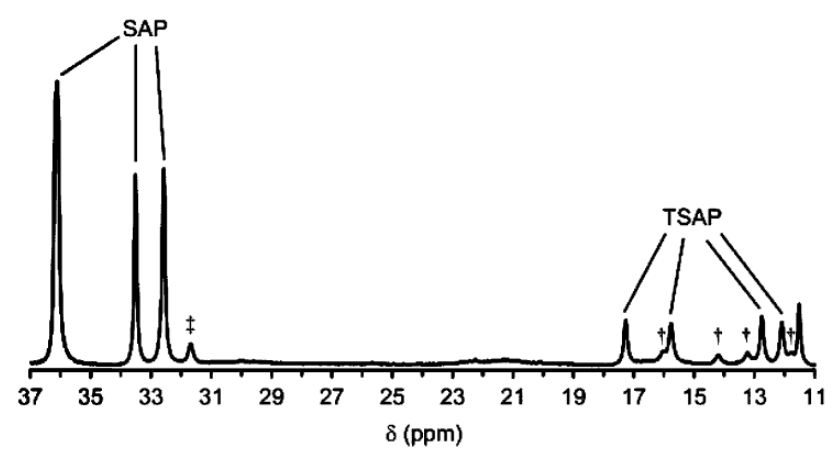

Figure 4.2: The most shifted axial proton $\left(a x^{S}\right)$ region of the ${ }^{1} \mathrm{H}$ NMR spectrum for the crude chelation product of EuNB-DOTA $\left(\mathrm{D}_{2} \mathrm{O}, \mathrm{pD} 5.5,400 \mathrm{MHz}\right.$, and $\left.283 \mathrm{~K}\right)$. Note there are two sets of SAP proton signals and two sets of TSAP proton signals, the minor species designated with $(\ddagger$ and $\dagger)$. 
Substitution of the DOTA ligand framework introduces a stereogenic center, indicating that the two enantiomeric pairs of LnDOTA chelates become four diastereoisomers. With the $S$ - configuration required of NB-DOTA the four possible diastereoisomeric chelates are: $S-\Delta(\lambda \lambda \lambda \lambda), S-\Delta(\delta \delta \delta \delta), S-\Lambda(\lambda \lambda \lambda \lambda)$, and $S-\Lambda(\delta \delta \delta \delta)$. One explanation for the observation of four chemically distinct chelates in solution is that all four diastereoisomers are present in solution. However, for this to be true the benzylic substituent would need to be able to occupy both axial and equatorial positions on the macrocyclic ring. This possibility can be eliminated based on previous EXSY studies that show inversion of the ring does not occur; the benzylic substituent can only occupy an equatorial position. ${ }^{72}$ This renders two of the diastereoisomers $(\Delta(\lambda \lambda \lambda \lambda)$ and $\Lambda(\lambda \lambda \lambda \lambda))$ inaccessible. This suggests that, in addition to the coordination isomerism, regioisomers similar to those observed in LnNB-DOTMA chelates (Chapter 3) are also present in the LnNB-DOTA chelates. To examine whether the additional peaks on the ${ }^{1} \mathrm{H}$ NMR spectra of LnNB-DOTA chelates were the result of regioisomerism of the nitrobenzyl group, preparatory reverse-phase HPLC was used to isolate any regioisomeric species present. ${ }^{113}$ 


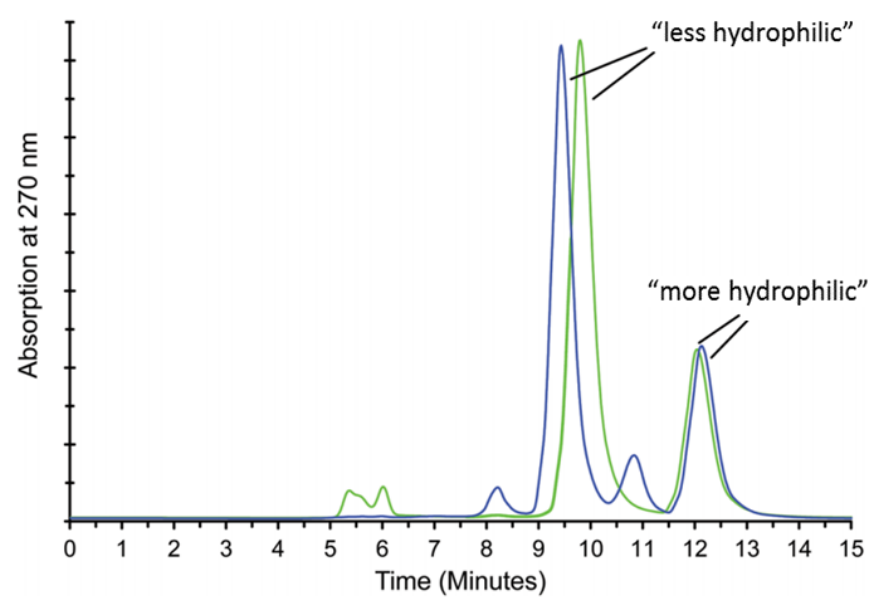

Figure 4.3: HPLC chromatograms of $\mathrm{Yb}^{3+}$ and EuNB-DOTA (green and blue, respectively).

Two isomeric chelates were obtained from the RP-HPLC analysis (Figure 4.3), as verified by mass spectrometric analysis. The ratio of the two isomeric chelates determined by RP-HPLC is close to a 2:1 ratio for each $\mathrm{Ln}^{3+}$ ion investigated (Table 4.1).

Table 4.1: Distribution of the structural isomers in LnNB-DOTA, as determined by RP-HPLC Chromatogram

\begin{tabular}{cc}
\hline Chelate & \% More Hydrophilic Species \\
\hline EuNB-DOTA & 68.4 \\
GdNB-DOTA & 78.23 \\
YbNB-DOTA & 71.7 \\
\hline
\end{tabular}

After RP-HPLC purification, the isolated species were probed by NMR. The ${ }^{1} \mathrm{H}$ NMR spectra reveal that each of the isolated products adopt both SAP and TSAP isomers in 
different relative abundances (Figure 4.4). This is a result of the design of the NB-DOTA ligand allowing the pendant arms to rotate, but preventing the ethylene bridges from inversion in $\mathrm{Ln}^{3+}$ chelates.
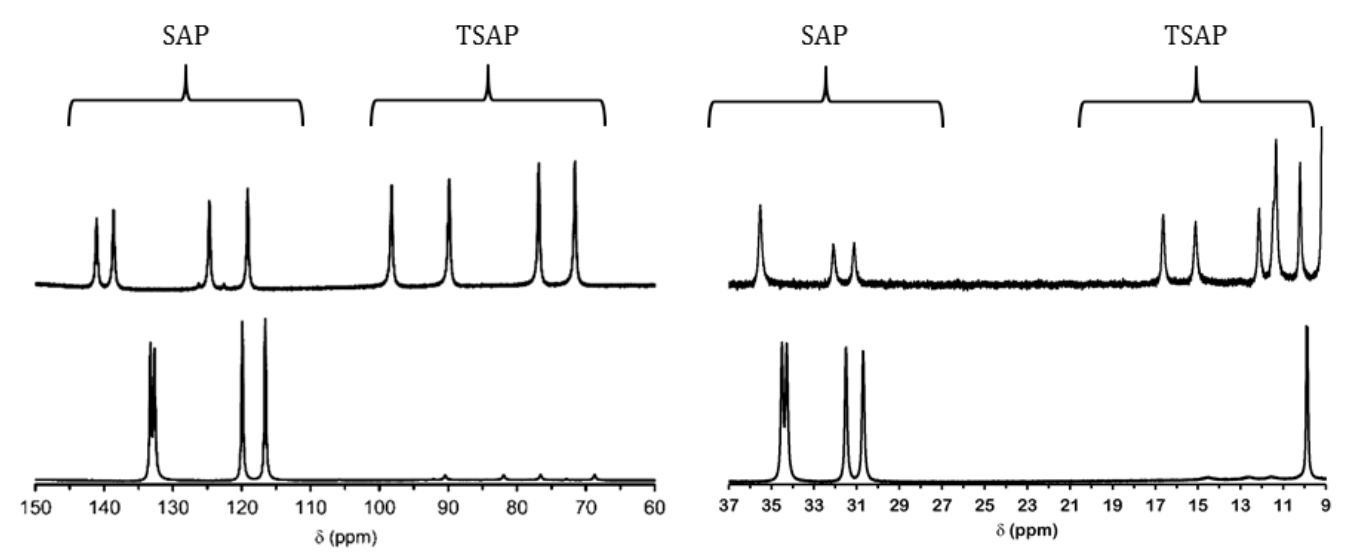

Figure 4.4: The most shifted axial proton $\left(a x^{\varsigma}\right)$ region ${ }^{1} \mathrm{H}$ NMR spectra $\left(\mathrm{D}_{2} \mathrm{O}, 400 \mathrm{MHz}, 283 \mathrm{~K}, \mathrm{pD} \sim 3.5\right)$ of the purified regioisomers of YbNB-DOTA (left) and EuNB-DOTA (right). The more hydrophilic species are shown on the top and the less hydrophilic species on the bottom. In the EuNB-DOTA spectra, some benzylic resonances are visible in the up field region shown.

The ${ }^{1} \mathrm{H}$ NMR spectra of these isomers for the $\mathrm{Yb}^{3+}$ and $\mathrm{Eu}^{3+}$ chelates of NB-DOTA show complicated proton signals for the 23 unique ligand protons. The relative populations of the two geometric isomers in solution can be obtained by line fitting of the most shifted axial protons in Figure 4.4. For both the $\mathrm{Yb}^{3+}$ and $\mathrm{Eu}^{3+}$ chelates of NB-DOTA the ${ }^{1} \mathrm{H}$ spectra show that the more hydrophilic (major) isomer has a higher predominance of SAP geometry while the less hydrophilic (minor) isomer has a predominance of TSAP geometry (Table 4.2). 
Table 4.2: Distribution of the geometric isomers in the purified structural isomer solutions of $\mathrm{Eu}^{3+}$ and YbNB-DOTA

\begin{tabular}{ccc}
\hline Chelate & Retention time (mins) & \% SAP \\
\hline EuNB-DOTA & 9.43 & 84.9 \\
& 12.13 & 40.1 \\
\hline YbNB-DOTA & 9.80 & 97.6 \\
& 12.05 & 41.3 \\
\hline
\end{tabular}

From the ${ }^{1} \mathrm{H}$ NMR spectra of the two isolated structural isomers of $\mathrm{Yb}^{3+}$ and $\mathrm{Eu}^{3+}$ chelates of NB-DOTA, it is apparent that assignment of the major SAP and TSAP chelates in the crude chelation solution as arising from the same chelate was incorrect. Instead the more hydrophilic chelate gives rise to the major SAP species and minor TSAP species seen in the ${ }^{1} \mathrm{H}$ spectrum of the crude chelation product. And the less hydrophilic chelate gives rise to the major TSAP species and minor SAP species seen in the crude chelation product.

\subsection{Structural Isomers of LnNB-DOTA}

\subsubsection{Isomer identification}

Structural and dynamic information about LnNB-DOTA can be elucidated by ${ }^{1} \mathrm{H}-{ }^{1} \mathrm{H}$ COSY and ${ }^{1} \mathrm{H}-{ }^{1} \mathrm{H}$ EXSY 2D NMR analysis. In ${ }^{1} \mathrm{H}-{ }^{1} \mathrm{H}$ COSY spectra the cross-peaks provide information about $J$-couplings - the magnitude of which can reveal relative orientation of protons in cyclic structures. The strongest $J$-couples in these spectra are 
geminal couplings (protons on the same carbon). These are followed by vicinal couples (protons on neighboring carbons) in which the protons are either anti-periplanar or eclipsed $\left(0^{\circ}\right.$ or $\left.180^{\circ}\right)$, as given by the Karplus relationship, i.e. $a x^{\mathrm{S}}-a x^{\mathrm{C}}$ couples (Figure 4.5). The $a x-e q$ vicinal $J$-couples are the smallest in magnitude owing to the $60^{\circ}$ angle between the two protons.

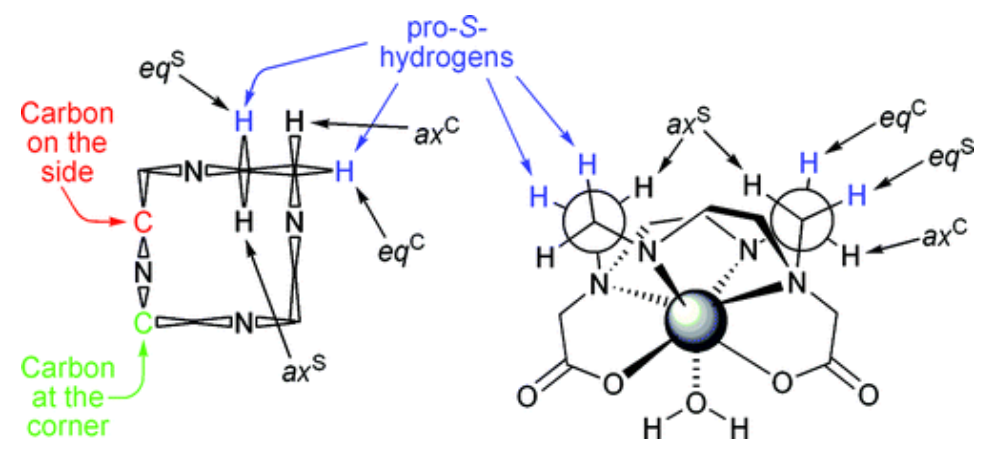

Figure 4.5: Locations of corner and side carbons in the [3333] ring (left) and the relative positioning of the protons of the chelate (right) for proton identification by ${ }^{1} \mathrm{H}-{ }^{1} \mathrm{H}$ COSY spectra data.

In LnDOTA-type chelates assignment of the NMR spectrum is most easily accomplished by starting with the most down-field shifted proton signals: the axial side $\left(a x^{\mathrm{S}}\right)$ protons. Their shift is a result of their relative positioning on the chelate and proximity to the paramagnetic metal center (Figure 4.5). Starting from the $a x^{\mathrm{S}}$ proton resonances and working through the various couplings, all the macrocyclic protons can be identified. However, it is impossible to definitively determine which ethylene bridge or pendant arm is which, since ${ }^{1} \mathrm{H}-{ }^{1} \mathrm{H}$ coupling does not pass through the amines of the macrocycle. The only readily identifiable ethylene bridge is the one with the benzylic substituent. It is identified by the absence of an equatorial proton resonance and the cross-peaks 
associated with it. If the benzylic group is on the side carbon the bridge will have three $a x^{\mathrm{S}}-e q^{\mathrm{S}}$ couples and four $a x^{\mathrm{C}}-e q^{\mathrm{C}}$ couples. If the benzylic group is on the corner carbon it will have four $a x^{\mathrm{S}}-e q^{\mathrm{S}}$ couples and three $a x^{\mathrm{C}}-e q^{\mathrm{C}}$ couples; the missing equatorial proton suggests the position of the benzylic group. The axial proton missing a fourth geminal coupling will instead show a vicinal coupling with benzylic protons; this conclusively indicates the presence of the benzylic group. The identification of benzylic protons in these chelates is assisted by the presence of couples to the aromatic protons. The aromatic protons are readily identified by their integration (twice that of the other proton resonances in the spectrum) and their characteristic shifts. 


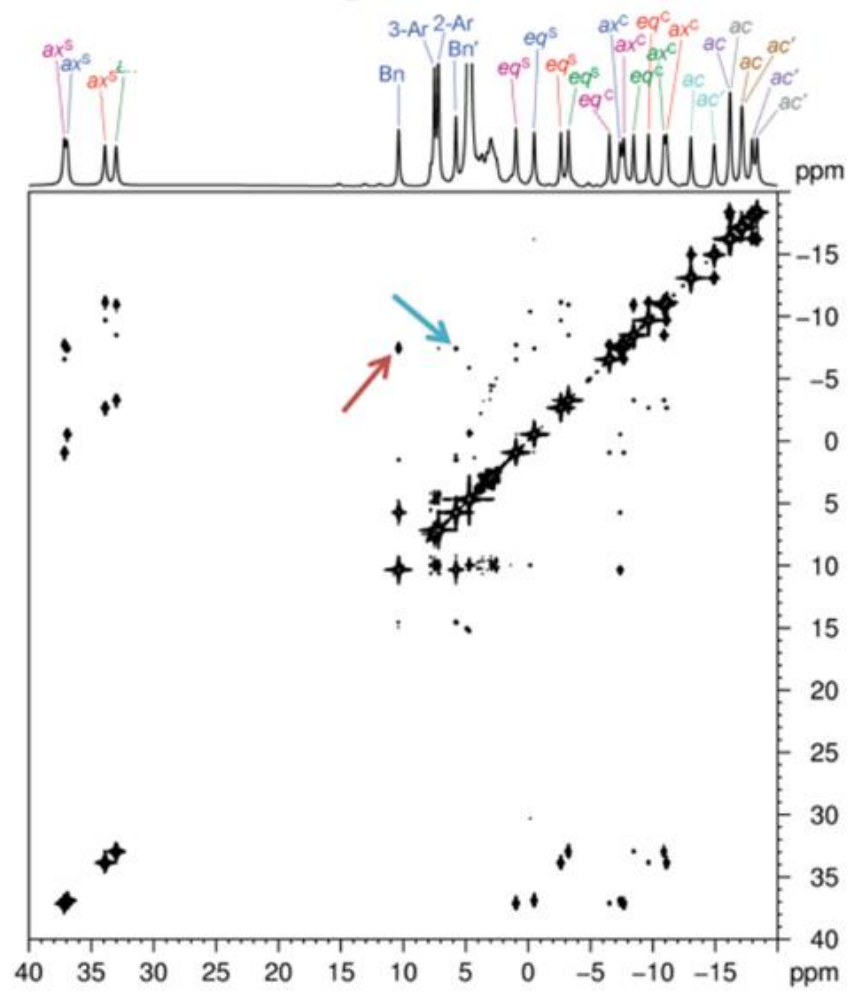

Figure 4.6: ${ }^{1} \mathrm{H}-{ }^{1} \mathrm{H}$ COSY spectrum of the more hydrophilic (corner) EuNB-DOTA isomer at $400 \mathrm{MHz}$ and $283 \mathrm{~K}$. The blue and red arrows show interactions between $B n \leftrightarrow a x^{C}$ and $B n^{\prime} \leftrightarrow a x^{C}$ protons

The assignment of the more hydrophilic isomer of EuNB-DOTA is fairly straight forward due to it being approximately $95 \%$ SAP geometry (Figure 4.6). Starting from the $a x^{S}$ protons, four germinal couplings to equatorial side $\left(e q^{\mathrm{S}}\right)$ protons can be readily identified; this indicates that this regioisomer has the benzylic group located on the corner of the macrocycle. Further support for this conclusion is provided by the presence of only three $a x^{\mathrm{C}}-e q^{\mathrm{C}}$ couples. Instead of a fourth there is a coupling of the $a x^{\mathrm{C}}$ proton with the $B n$ and $B n^{\prime}$ protons. Vicinal axial and equatorial protons on the bridge with the benzylic group have stronger coupling than seen between these protons on other bridges as a result 
of distortions from gauche conformation to accommodate the bulky substituent. From these data, all of the chelate protons in the SAP isomer of the corner regioisomer were assigned (Figure 4.6).

A similar analysis is possible with the less hydrophilic isomer of EuNB-DOTA. However, interpretation of this spectrum is made more difficult by the presence of almost equimolar quantities of SAP and TSAP isomers. Starting from the most defined resonances $\left(a x^{S}\right)$ we looked for geminal and vicinal couples: for each of the TSAP and SAP isomers there are three strong couples of similar strength, four weaker couples of similar strength, and one of a relative intermediate strength. The three strongest couples are geminal $a x^{\mathrm{S}}-e q^{\mathrm{S}}$ couples, the four weaker couples are vicinal $a x^{\mathrm{S}}-a x^{\mathrm{C}}$ couples, and the intermediate strength couple is a vicinal couple for $a x^{S}-B n^{\prime}$. The resonance of the benzylic proton in this vicinal couple (for both the SAP and TSAP isomers) is shifted upfield of the water resonance as a result of its relative position to the $\mathrm{Eu}^{3+}$ ion. The $a x^{\mathrm{S}}-$ $B n^{\prime}$ cross-peaks for both the SAP and TSAP isomers of this spectrum confirm the assignment of side isomer to the less hydrophilic regioisomer. 


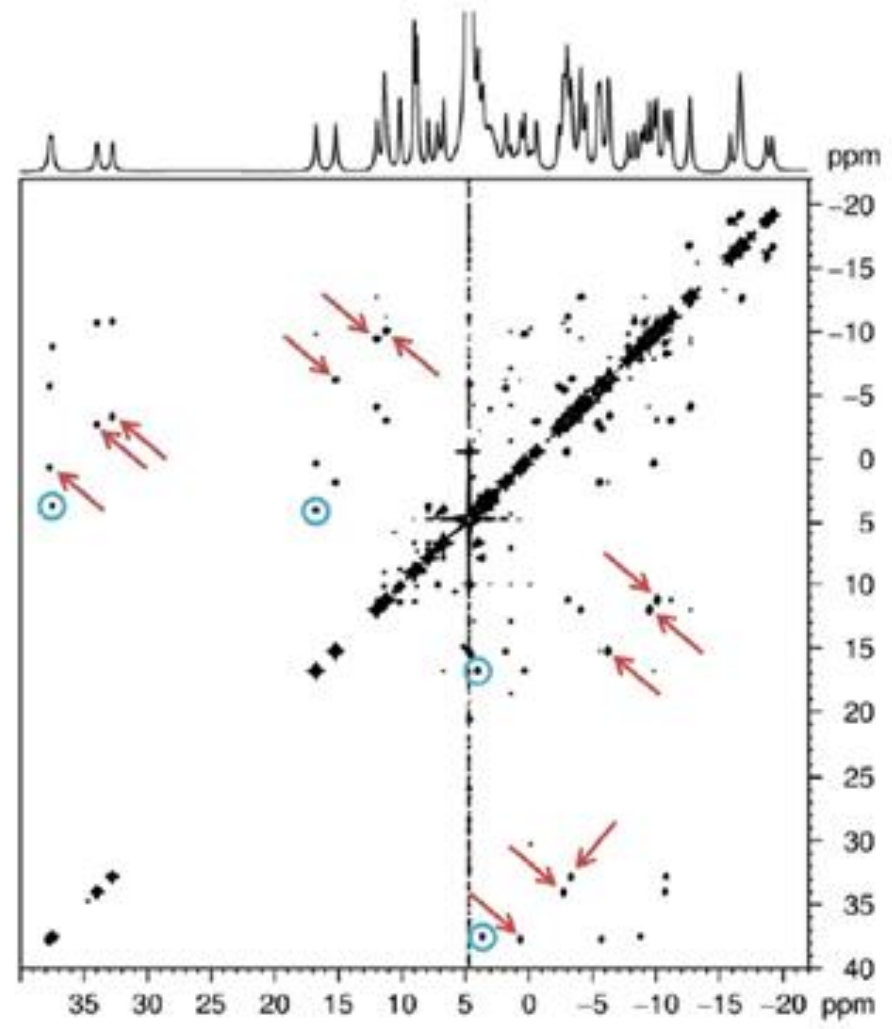

Figure 4.7: ${ }^{1} \mathrm{H}-{ }^{1} \mathrm{H}$ COSY spectrum of the less hydrophilic (side) EuNB-DOTA isomer at $400 \mathrm{MHz}$ and $283 \mathrm{~K}$. The interactions between the $B n^{\prime} \leftrightarrow a x^{\mathrm{s}}$ and $a x^{S} \leftrightarrow e q^{S}$ protons of both geometric isomers are indicated with blue circles and red arrows, respectively.

The identification of the less hydrophilic species of EuNB-DOTA as the side isomer was confirmed by analysis of the macrocyclic ring and benzylic proton resonances in the ${ }^{1} \mathrm{H}-$ ${ }^{1} \mathrm{H}$ COSY data (Figure 4.7). However, the complete assignment of the protons of the side regioisomer is complicated by the geometric isomer distribution that causes considerable overlap of the up-field proton resonances. ${ }^{1} \mathrm{H}-{ }^{1} \mathrm{H}$ EXSY data elucidates these more difficult assignments of EuNB-DOTA chelate protons (Figure 4.8). The complete 
assignment of the chelate protons for both the TSAP and SAP species was accomplished by careful consideration of the COSY and EXSY 2D NMR data in tandem.

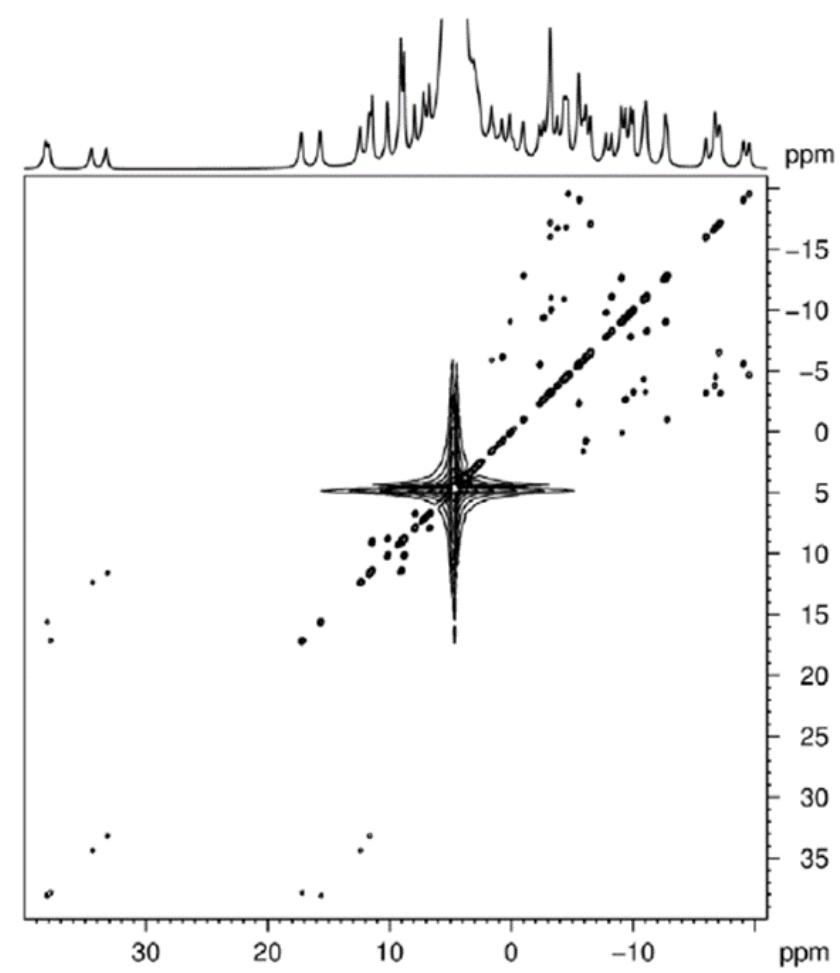

Figure 4.8: ${ }^{1} \mathrm{H}-{ }^{1} \mathrm{H}$ EXSY spectrum of the side EuNB-DOTA isomer at $400 \mathrm{MHz}$ and $283 \mathrm{~K}$.

The cross-peaks in ${ }^{1} \mathrm{H}-{ }^{1} \mathrm{H}$ EXSY spectra show which protons exchange with one another during a mixing period; this allows us to see exchange between two species in solution. For example, the cross-peaks of the EXSY spectra in our chelates indicate that the $a x^{S}$ proton in the SAP isomer exchanges with the $a x^{\mathrm{S}}$ proton in TSAP geometry, indicating that SAP-TSAP exchange can occur, but that it does so without conformational changes to the macrocycle. This conclusion agrees with the findings of the 2004 paper. $^{72}$ By combining the information provided by the EXSY spectrum with that available from the 


\section{CHAPTER 4}

COSY spectrum, a complete proton assignment of the SAP and TSAP species in the side isomer was accomplished (Figure 4.9).

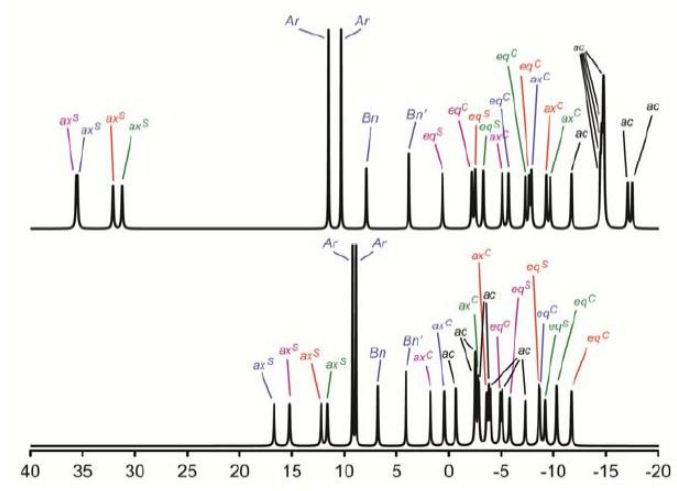

Figure 4.9: Simulated ${ }^{1} \mathrm{H}$ NMR spectra for the SAP (top) and

TSAP (bottom) protons of the side isomer with assignments showing relative ethylene bridges by color.

Completing the proton assignment for the minor (TSAP) isomer of the corner regioisomer is impossible because the proton resonances for this isomer are mostly obscured in the ${ }^{1} \mathrm{H}$ NMR spectrum. For this reason the additional data obtained in the ${ }^{1} \mathrm{H}-$ ${ }^{1} \mathrm{H}$ EXSY spectrum (Figure 4.10) were not beneficial for further elucidation of the minor species, so the proton resonances of the corner isomer TSAP species remain unassigned. 


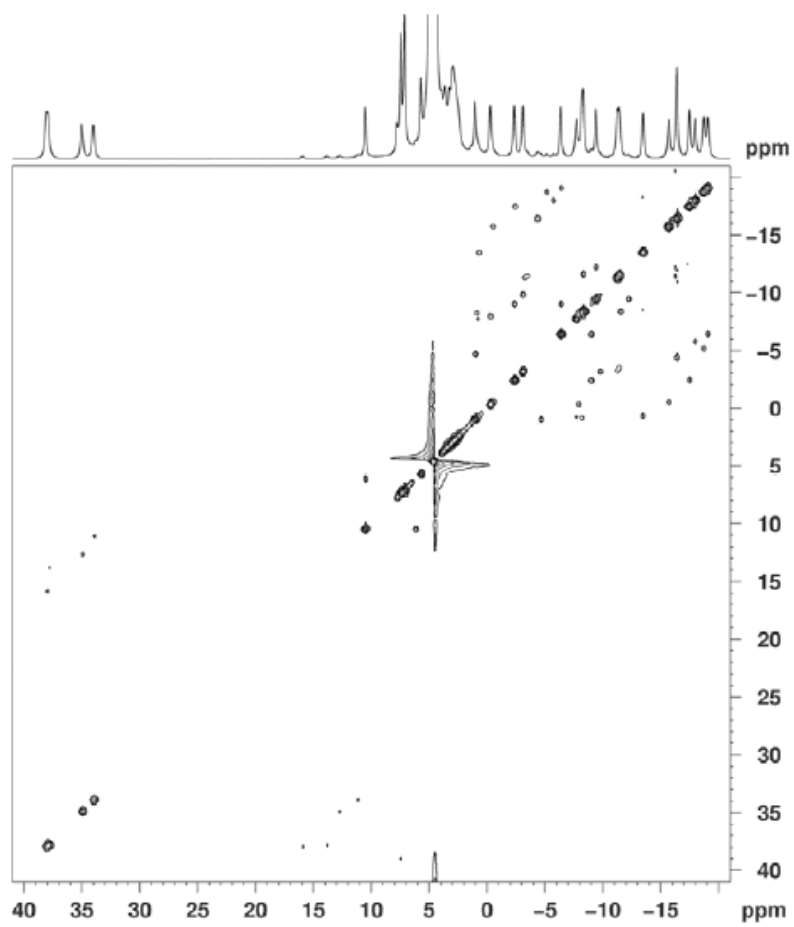

Figure 4.10: ${ }^{1} \mathrm{H}-{ }^{1} \mathrm{H}$ EXSY spectrum of the corner EuNB-DOTA isomer at $400 \mathrm{MHz}$ and $283 \mathrm{~K}$.

\subsubsection{Orientation of the benzylic group}

The orientation of the benzylic groups in the related EuNB-DOTMA chelates were determined to be non-equivalent between the regioisomers by nuclear Overhauser effect (nOe) peaks seen in the ${ }^{1} \mathrm{H}-{ }^{1} \mathrm{H}$ COSY data. This type of peak will occasionally be seen in ${ }^{1} \mathrm{H}-{ }^{1} \mathrm{H}$ COSY spectra; they are a result of dipolar interactions that occur through space, rather than through bonds. However, in the EuNB-DOTA regioisomers the cross-peaks seen in the ${ }^{1} \mathrm{H}-{ }^{1} \mathrm{H}$ COSY spectra are all attributable to ${ }^{3} J$-couplings between the benzylic protons and an axial proton. The aromatic protons of both regioisomers show no crosspeaks in the ${ }^{1} \mathrm{H}-{ }^{1} \mathrm{H}$ COSY spectra with macrocyclic or pendant arm protons. 


\section{CHAPTER 4}

In the corner isomer there are ${ }^{1} \mathrm{H}-{ }^{1} \mathrm{H}$ COSY couplings between both benzylic protons and the $a x^{\mathrm{C}}$ proton, while in the side isomer there is only a single couple seen between the upfield benzylic proton and the $a x^{\mathrm{S}}$ proton. These differences in the number of ${ }^{3} J$-couplings between the benzylic protons and an axial proton in the two regioisomers suggest different orientations for the benzylic substituent relative to the macrocycle. However, the absence of visible couples is insufficient evidence to conclusively state the orientations of the benzylic substituent in these chelates. The suggestion that the orientation of the benzylic groups is different on the two regioisomers of EuNB-DOTA is supported by differences in their benzylic proton resonance patterns. The corner isomer has one resonance that is largely unaffected by its proximity to the paramagnetic metal ion and another that is shifted substantially down-field. The side isomer displays hyperfine shifts for both benzylic protons: one shifted down-field and the other shifted significantly up-field. These relative shifts of the corner and side benzylic protons are similar to those of the related EuNB-DOTMA corner and side isomers. It seems reasonable to conclude, therefore, that the orientations of the benzylic substituent in corner and side isomers of LnNB-DOTA chelates are quite different.

\subsection{Interconversion of regioisomers}

High kinetic robustness and thermodynamic stability are important factors in selecting a BFC, as targeted agents are expected to spend extended time in the body. The kinetic and thermodynamic stabilities of GdNB-DOTA were determined in the 2004 publication: acid catalyzed dissociation was reported at $k_{\mathrm{D}}=6 \times 10^{-7} \mathrm{~s}^{-1}(1 \mathrm{mM}$ agent solutions incubated in $\mathrm{pH}=1 \mathrm{HNO}_{3}$ at $298 \mathrm{~K}$ ) and the thermodynamic stability was reported at 
$K_{\mathrm{GdL}}=24.2\left(1.0 \mathrm{M} \mathrm{Me}{ }_{4} \mathrm{NCl}\right.$ solutions at $\left.298 \mathrm{~K}\right)$. The thermodynamic stability is determined at chelation, so there is no way to separate the contributions of the regioisomers. The acid catalyzed dissociation study was completed on the crude reaction product, not on isolated regioisomers. ${ }^{72}$

It was found in other rigid chelates with benzylic substituted macrocycles that interconversion between corner and side isomers is not possible through conformational changes. ${ }^{67,68}$ However, the rigid single isomer can be converted to a mixture of isomers under acidic conditions that enable the metal ion to dechelate and recombine. ${ }^{68,74}$ The corner and side isomers of LnNB-DOTMA and LnNB-DO3MA-1A chelates were shown to interconvert after incubation at high temperatures under acidic conditions and not undergo acid-catalyzed epimerization allowing for exchange from SAP to TSAP geometries. Thermodynamic stability constants for the $\mathrm{YbS}$-SSS-NB-DO3MA-1A and $\mathrm{YbS-RRRR-NB-DOTMA}$ chelates were determined to be $K_{\mathrm{YbL}}=21.91$ and 24.12 , respectively $(1.0 \mathrm{M} \mathrm{KCl}$ at $298 \mathrm{~K}) .{ }^{68}$ The slow rate (10 weeks to reach equilibrium) of interconversion between regioisomers speaks to the kinetic robustness of these chelates the stability constant of the clinically approved GdDOTA is often used for comparison $\left(K_{\mathrm{GdL}}=24.7\right){ }^{72}$

To consider the interconversion of regioisomers of the YbNB-DOTA corner isomer we incubated the purified corner isomer at $90{ }^{\circ} \mathrm{C}$ and $\mathrm{pD} \sim 1$ (Figure 4.11). After 1 week of incubation there was significant growth of side isomer - only $78.9 \%$ of the chelate remained in the corner isomer. There was no further development of side isomer after 2 weeks of incubation. Since conformational rearrangement of the corner isomer to the 
side isomer is impossible (see ${ }^{1} \mathrm{H}-{ }^{1} \mathrm{H}$ EXSY data, Figures 4.8 and 4.10) this interconversion must occur through the dissociation and rechelation of the metal ion. The kinetics of this interconversion is determined by constants for both the dechelation and recombination.

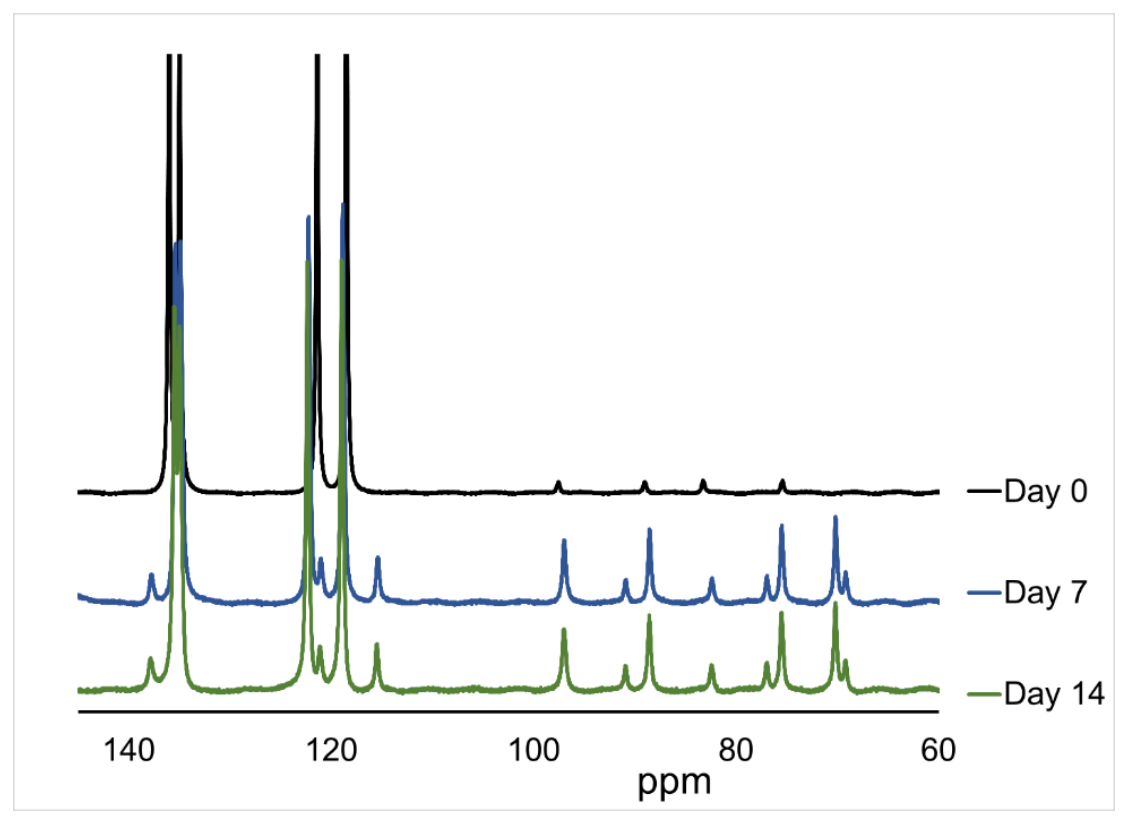

Figure 4.11: The most shifted axial proton region ${ }^{1} \mathrm{H}$ NMR spectra of purified YbNB-DOTA corner isomer before and after incubation $\left(\mathrm{D}_{2} \mathrm{O}, \mathrm{pD} 1,600 \mathrm{MHz}\right.$, and $\left.298 \mathrm{~K}\right)$.

Equilibrium is achieved much more rapidly in the case of YbNB-DOTA - equilibrium was established in less than a week (Figure 4.11). This indicates that the rate constants for interconversion between LnNB-DOTA regioisomers are significantly smaller than those of LnNB-DOTMA and LnNB-DO3MA-1A. This is attributable to the methyl substituents of the pendant arms present in both of the LnNB-DOTMA and LnNB- 
CHAPTER 4

DO3MA-1A chelates - but absent in LnNB-DOTA - that render the arms more rigid and presumably decreases the propensity for dechelation of the metal ion.

\subsection{GdNB-DOTA: How does regioisomerism effect NB-DOTA as a contrast agent?}

To determine the water exchange kinetics of the GdNB-DOTA chelates, the transverse ${ }^{17} \mathrm{O}$ relaxation rate constants $\left(R_{2 \mathrm{p}}\right)$ were measured as a function of temperature (Figure 4.13). The ${ }^{17} \mathrm{O}$ NMR data were collected by the laboratory of Prof. Botta at the University of Eastern Piedmont in Alessandria, Italy. Inspection of the $R_{2 \mathrm{p}} v s$. temperature data provides a qualitative assessment of the relative rates of exchange (Figure 4.12). If it were possible to get all exchange rates from a single chelate, the $R_{2 \mathrm{p}}$ $v s$. temperature profile would display a large bell curve: a peak is formed as the increase in temperature speeds water exchange rates from very slow to very fast, and the maximum reveals the optimal transverse relaxation with moderate-fast exchange.

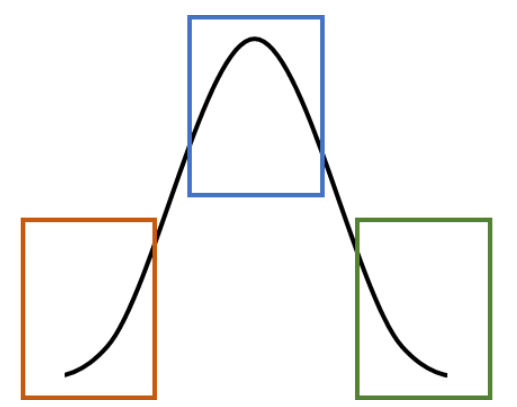

Figure 4.12: The bell curve formed from transverse ${ }^{17} \mathrm{O}$ relaxation rates vs. temperature plots with a full range of water exchange rates: slow (orange), intermediate (blue), and fast (green).

Chelates in TSAP geometry typically have very fast water exchange relative to analogous SAP chelates. Solutions with significant contributions from both SAP and TSAP isomers 


\section{CHAPTER 4}

are likely to produce ${ }^{17} \mathrm{O}$ transverse relaxation profiles that exhibit features of both fast and intermediate exchange regimes. This allows $\tau_{\mathrm{M}}$ values to be determined for both the SAP and TSAP isomers from the same ${ }^{17} \mathrm{O}$ relaxation profile. In these cases the distribution of the TSAP and SAP isomers for the $\mathrm{Eu}^{3+}$ chelates is used to independently fit the contributions of both species to the transverse ${ }^{17} \mathrm{O}$ relaxation. ${ }^{114}$ However, when exchange from one isomer dominates the ${ }^{17} \mathrm{O}$ relaxation profile, only a single value of $\tau_{\mathrm{M}}$ can be obtained from the fitting - representing an inherent weighted average of the $\tau_{M}$ values of the two isomers in solution.

For GdNB-DOTA regioisomers the geometric isomer distribution is assumed to be consistent with those of the EuNB-DOTA regioisomers on the basis of the similarly sized metals. This means that the corner isomer has $95 \%$ SAP geometry and the side isomer has $45 \%$ SAP geometry in solution. The ${ }^{17} \mathrm{O}$ transverse relaxation profiles for the regioisomers of GdNB-DOTA are seen in Figure 4.13. Despite the significant differences in geometric isomer distributions, both plots show maxima indicating moderately fast water exchange kinetics.
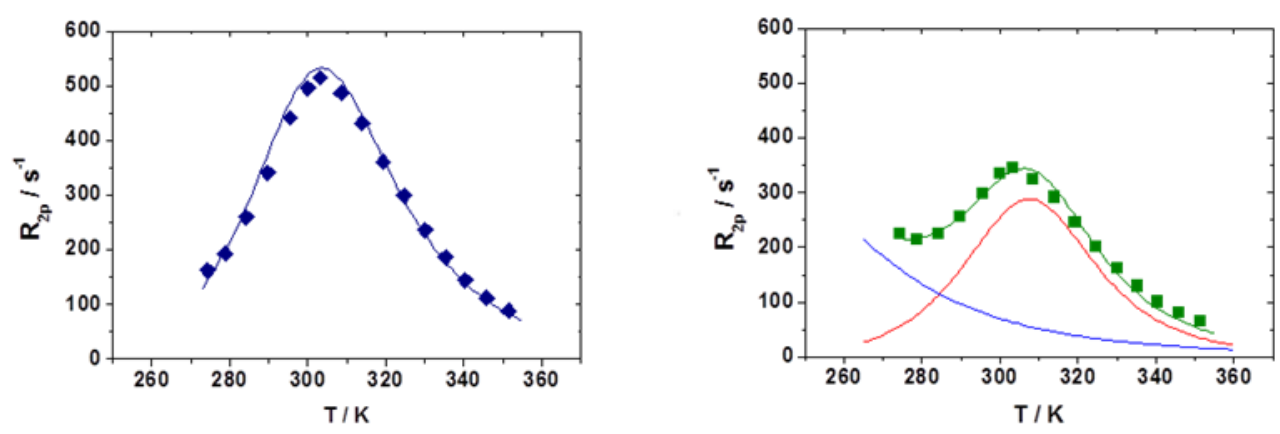

Figure 4.13: Transverse ${ }^{17} \mathrm{O}$ relaxation rates as a function of temperature for corner (left) and side (right) GdNB-DOTA at $\mathrm{pH}=6.5$ and $11.74 \mathrm{~T}$. 
The TSAP isomer of the corner regioisomer does not noticeably contribute to the shape of the ${ }^{17} \mathrm{O}$ transverse relaxation curve. Fitting of these data therefore affords a $\tau_{\mathrm{M}}$ value that represents the weighted average of the SAP and TSAP isomers in solution. For the corner regioisomer, a $\tau_{\mathrm{M}}$ value of $188 \mathrm{~ns}$ was determined.

The ${ }^{17} \mathrm{O} R_{2 p}$ profile of the side isomer does not resemble a simple bell curve. Instead it has the appearance of two bell curves (representing very different exchange regimes) superimposed upon each other. This allows the contribution of the SAP and TSAP isomers to be determined separately by fitting the data to a model incorporating exchange from two chelates. The side TSAP isomer was determined to have a $\tau_{\mathrm{M}}$ value of $7 \mathrm{~ns}$ and the side SAP isomer a $\tau_{M}$ value of $250 \mathrm{~ns}$. From these values the weighted average $\tau_{\mathrm{M}}$ value is calculated to be $116 \mathrm{~ns}$ for the side isomer, reflecting the greater proportion of TSAP isomer present in solution.

Both of the regioisomers of GdNB-DOTA exhibit faster water exchange than the parent chelate GdDOTA ( $\tau_{\mathrm{M}}=244 \mathrm{~ns}$ ), which adopts $80 \%$ SAP geometry in solution; this is common for benzylic substituted macrocyclic chelates. ${ }^{115}$ Although water exchange is faster for the side isomer than the corner isomer, both regioisomers of GdNB-DOTA are in the fast exchange regime for low molecular weight chelates where $\tau_{R}$ limits relaxivity.

To obtain information about the parameters that influence relaxivity, such as $\tau_{\mathrm{R}}$, Nuclear Magnetic Relaxation Dispersion (NMRD) profiles are fit to the SBM equations. ${ }^{1} \mathrm{H}$ NMRD profiles record the longitudinal proton relaxivity of a contrast agent as a function

of the applied magnetic field strength $\left(B_{0}\right)$. The ${ }^{1} \mathrm{H}$ NMRD profiles of both isomers of 


\section{CHAPTER 4}

GdNB-DOTA are typical of low molecular weight chelates (Figure 4.14); relaxivity is high and flat in the low field region, and decreases gradually with increasing $\mathrm{B}_{0}$ field after dispersing above $1 \mathrm{MHz}$.

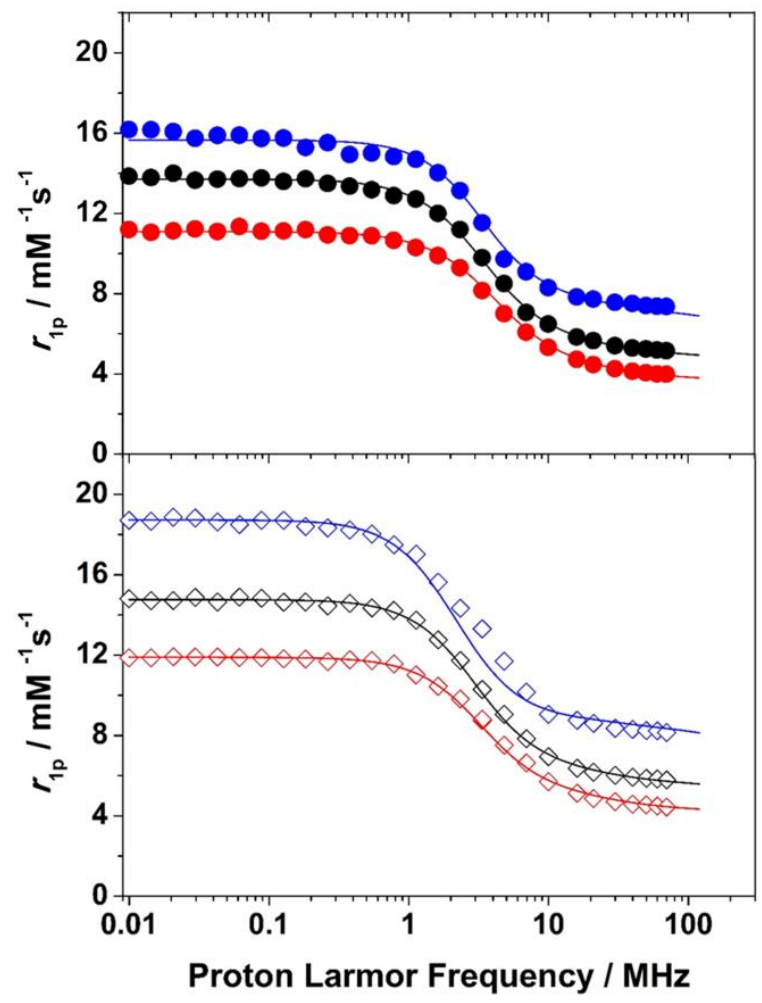

Figure 4.14: ${ }^{1} \mathrm{H}$ NMRD profiles of the corner (top) and side (bottom) regioisomers of GdNB-DOTA at 283, 298, and $310 \mathrm{~K}$ (blue, black, and red, respectively).

It is immediately apparent that the side isomer has higher relaxivity than the corner isomer. This indicates that the reduced hydration of the TSAP isomers arising from very fast water exchange kinetics ${ }^{64}$ - expected to have a greater negative impact on the relaxivity of the side isomer (55\% TSAP geometry) than the corner isomer (5\% TSAP geometry) - is counteracted by another parameter. In the low molecular weight chelates, tumbling in solution is commonly the limiting parameter for relaxivity. In light of the 
results obtained for the analogous GdNB-DOTMA chelates, which indicated that the side isomer tumbles more slowly in solution, it is not altogether surprising that the GdNB-DOTA side isomer has higher relaxivity. If the same situation for tumbling of the regioisomers prevailed for GdNB-DOTA then this would tend to increase relaxivity for the side isomer.

From the ${ }^{1} \mathrm{H}$ NMRD profiles it can be seen that relaxivity increases with decreasing temperature - this is typical of low molecular weight chelates in the fast exchange regime. As the temperature is reduced, the viscosity of the solution increases causing longer $\tau_{R}$ values, which in turn increases relaxivity. It is notable that the effect of changing temperature is more pronounced on the side isomer than the corner isomer (Figure 4.14). This is most apparent at low fields where relaxivity is governed by electron spin relaxation time constants and the rate of molecular tumbling. The more pronounced effect of temperature on the side isomer is consistent with the idea that this chelate is tumbling more slowly in solution.

The GdNB-DOTA ${ }^{1} \mathrm{H}$ NMRD data for both regioisomers were fit using a single species approximation (Table 4.3). The distribution of geometric isomers is accounted for in the fitting of the ${ }^{1} \mathrm{H}$ NMRD profiles both by using the weighted averages for $\tau_{\mathrm{M}}$ and by using a weighted average of $r_{\mathrm{GdH}}{ }^{\mathrm{SAP}}=3.0 \AA$ and $r_{\mathrm{GdH}}{ }^{\mathrm{TSAP}}=3.1 \AA$. These different $r$ values reflect different hydration states $\left(q / r^{6}\right)$ of the geometric isomers. This is supported by the ${ }^{17} \mathrm{O}$ NMR data indicating very fast water kinetics in the TSAP species of the side regioisomer of GdNB-DOTA. A reduced hydration in the species is expected as $\tau_{\text {off }}$ becomes significant with very fast water exchange kinetics . 
Table 4.3: Fitted parameters for the NMRD profiles of GdNB-DOTA at $298 \mathrm{~K}$.

a) fitting used $a=4.0 \AA,{ }^{298} D=2.24 \times 10^{-5} \mathrm{~cm}^{2} \mathrm{~s}^{-1}$ and $q=1$. b) fixed parameter.

\begin{tabular}{ccc}
\hline Parameter & Corner & Side \\
\hline$\tau_{\mathrm{M}}(\mathrm{ns})^{\mathrm{b}}$ & 188 & 116 \\
$\tau_{\mathrm{R}}(\mathrm{ps})$ & 90 & 120 \\
$\Delta H_{\mathrm{R}}\left(\mathrm{kJ} \mathrm{mol}{ }^{-1}\right)$ & 17 & 16 \\
$\tau_{\mathrm{V}}(\mathrm{ps})$ & 19.7 & 18 \\
$\Delta H_{\mathrm{V}}\left(\mathrm{kJ} \mathrm{mol}{ }^{-1}\right)$ & 1.0 & 1.0 \\
$\Delta^{2}\left(\times 10^{19} \mathrm{~s}^{-2}\right)$ & 0.86 & 1.2 \\
$r_{\mathrm{GdH}}(\AA)^{\mathrm{b}}$ & 3.005 & 3.055 \\
\hline
\end{tabular}

The fitted SBM parameters (Table 4.3) support the assertion that the side isomer tumbles much more slowly than the corner isomer in solution.

\subsection{Slowing rotation of GdBP-DOTA}

\subsubsection{GdBP-DOTA: Can regioisomerism influence chelate binding?}

The variable temperature ${ }^{1} \mathrm{H}$ NMRD data, and the fitting of the $298 \mathrm{~K}$ data, indicate that the regioisomers of GdNB-DOTA may have different self-association affinities. This has implications for the functionality of the NB-DOTA derivatives as BFCs. To determine the impact of this, IB-DOTA was functionalized to its biphenyl derivative (BP-DOTA); this preparation was based on previously published procedures for similar ligands and assisted by Lauren Rust (Scheme 4.1). ${ }^{28}$ The nitrobenzyl group was reduced by catalytic 
hydrogenation to the corresponding amine; the amine was then converted to the isothiocyanate by reaction with thiophosgene; the isothiocyanate readily undergoes coupling to 4-phenylbenzylamine, producing the functionalized biphenyl ligand, and the functionalized ligand was subsequently chelated with $\mathrm{Gd}^{3+}$ to generate a molecular probe. After chelation of the biphenyl ligand with $\mathrm{Gd}^{3+}$ the corner and side complexes were isolated by preparative HPLC. For identification purposes these $\mathrm{Gd}^{3+}$ complexes were synthesized and purified in parallel with the comparable $\mathrm{Eu}^{3+}$ complexes.

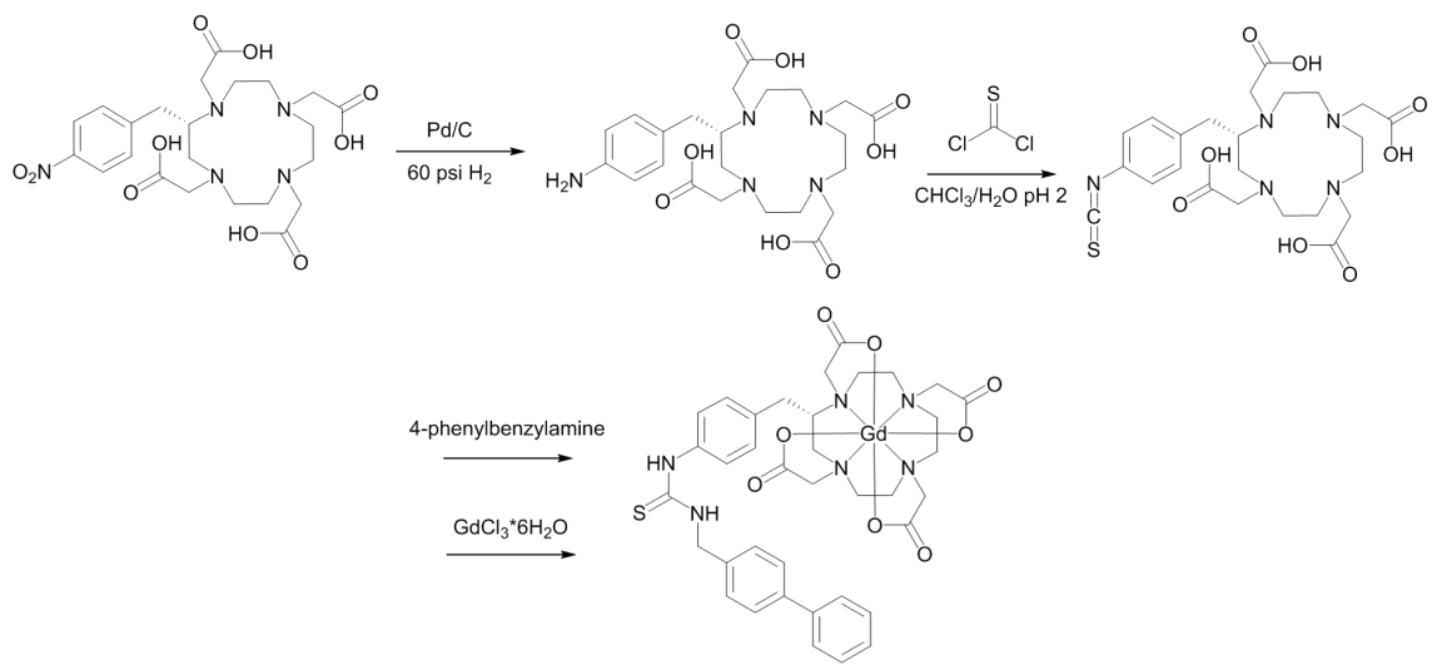

\section{Scheme 4.1: Synthesis of NB-DOTA biphenyl derivatives.}

The biphenyl moiety allows for the tumbling of these complexes to be slowed in solution through non-covalent interactions of the hydrophobic biphenyls, either by the formation of micelles or non-specific binding of the biphenyl moiety to a large macromolecule, such as poly- $\beta$-cyclodextrin (poly- $\beta-\mathrm{CD}){ }^{28}$ Slowing chelate tumbling lifts the limiting effect of $\tau_{R}$ and allows the impact of differing $\tau_{M}$ values in the corner and side isomers to be effectively probed. The concept of tumbling is more complicated for chelates slowed 
by the binding of a functional moiety than it is for free chelates. The tumbling of a bound chelate is coupled to the global motion of the bound object. However, this coupling may not necessarily be complete. If the chelate is able to freely rotate around the point of attachment, its molecular tumbling is then divided into global and local rotational components as proposed by Lipari and Szabo. ${ }^{116,}{ }^{117}$ Global rotation reflects the reorientation rate of the entire bound chelate and is typically very slow, but local rotation reflects the re-orientation of the chelate independent of the macromolecule and can be much more rapid. A combination of the global and local rotation is observed in solutions with bound GdBP-DOTA chelates; separate contributions can be approximated when fitting NMRD profiles.

\subsubsection{Self-association of the biphenyl moiety}

Micelles are expected to form in the GdBP-DOTA system as a result of the sizable hydrophobic moiety on the opposite end of the compound from the hydrophilic water exchange site. ${ }^{28,48}$ Chelates aggregate to different extents until the solution concentration reaches the critical micelle concentration (CMC). ${ }^{118}$ Above this point it is expected that all chelates will be incorporated into a micelle. 

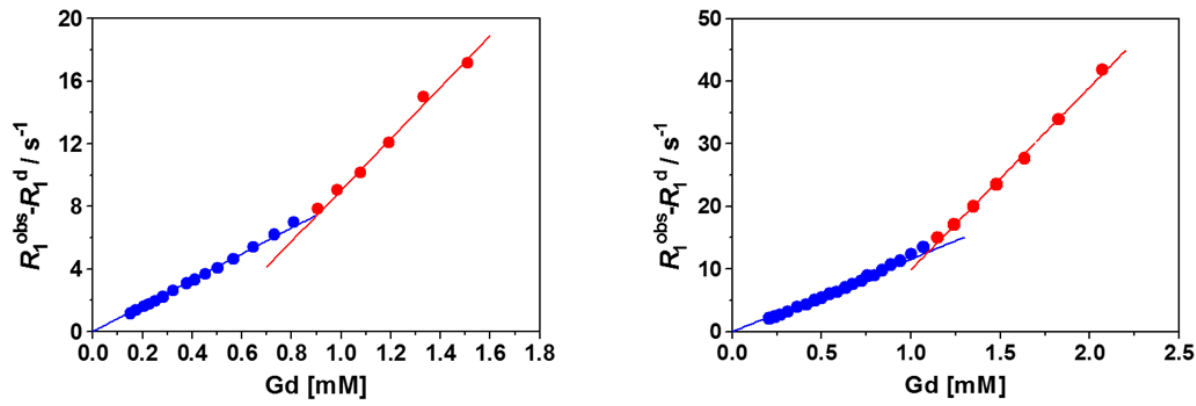

Figure 4.15: Titration of side and corner isomers of GdBP-DOTA to determine the critical micelle concentrations $(20 \mathrm{MHz}$ and $298 \mathrm{~K})$.

The longitudinal relaxation rate constant is normally linearly dependent upon the $\mathrm{Gd}^{3+}$ chelate concentration. However, when tumbling is slowed by incorporation of the chelates into micelles, the relaxation rate constant will increase leading to an inflection point at the CMC (Figure 4.15). This allows micelle formation to be monitored by relaxometric analysis with increasing concentrations of the complex. Once micelles have formed, their size can be determined by dynamic light scattering (Table 4.4).

Table 4.4: CMC and micelle size of corner and side GdBP-DOTA at $298 \mathrm{~K}$, as determined by relaxometric titration and DLS, respectively.

\begin{tabular}{ccc}
\hline Isomer & $\mathrm{CMC}(\mathrm{mM})$ & Size $(\mathrm{nm})$ \\
\hline Corner & 0.90 & 10 \\
Side & 1.10 & 18 \\
\hline
\end{tabular}

The side isomer requires slightly higher concentrations to form micelles, and the micelles formed are nearly twice the size of those formed by the corner isomer. This difference in 
self-association is consistent with other chelates containing benzylic substituted macrocycles, and is presumably the result of differing orientations of the benzylic substituent relative to the macrocycle.

To determine how the increase in $\tau_{\mathrm{R}}$ affects relaxivity ${ }^{1} \mathrm{H}$ NMRD profiles were collected for the free chelate and the micelles of GdBP-DOTA (Figure 4.16). The relaxometric data for the free biphenyl conjugate complex below the CMC resembles those of low molecular weight chelates. However, the ${ }^{1} \mathrm{H}$ NMRD profile of the GdBP-DOTA side isomer below the CMC shows a very small "hump" at high fields. This is indicative of a greater degree of aggregation in the side isomer than in the corner which exhibits no hump. The relaxometric data for GdBP-DOTA above the CMC reflects their slowed tumbling with an increased relaxivity across all fields and a characteristic hump at high fields. These effects are more pronounced for the side isomer, as compared with the corner isomer, consistent with the side regioisomer forming larger micelles, as determined by DLS. This in turn leads to a longer $\tau_{\mathrm{R}}$ value for the side regioisomer than for the corner regioisomer. 


\section{CHAPTER 4}
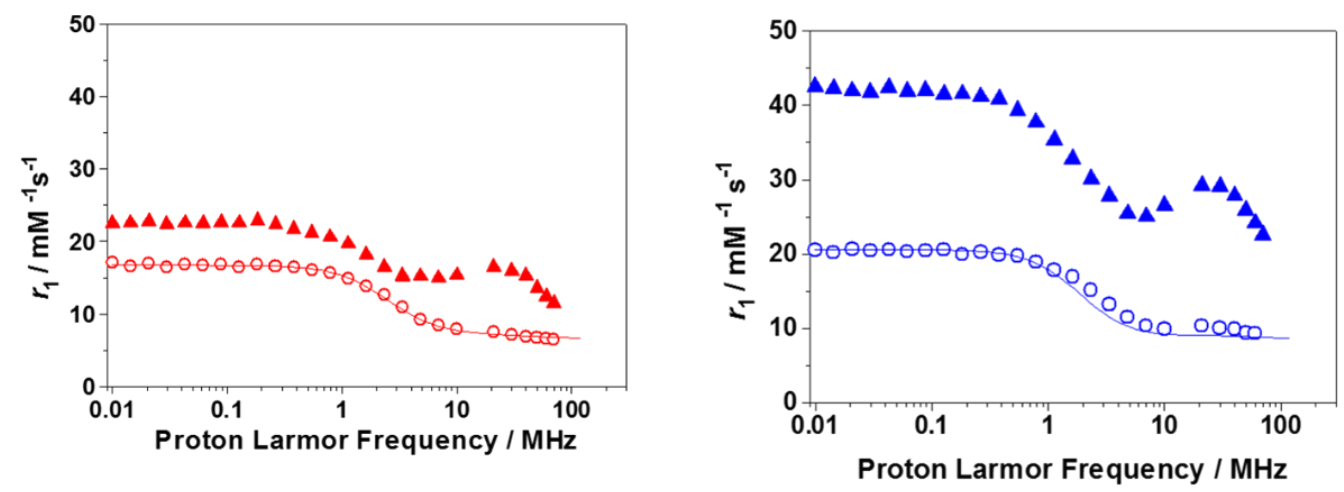

Figure 4.16: The NMRD profiles of free complex (open circles) and complex above the CMC limit (closed triangles) for the corner (left) and the side (right) isomers of GdBP-DOTA at $298 \mathrm{~K}(\mathrm{pH}=6.7$ at $0.15 \mathrm{mM}$ and $\mathrm{pH}=6.5$ at $0.20 \mathrm{mM}$, respectively).

As with the GdNB-DOTA chelates, the very fast water exchange kinetics of TSAP geometry could be expected to negatively impact the relaxivity of the side isomer of GdBP-DOTA: $55 \%$ of the side isomer and 5\% of the corner isomer. However, relaxivity of a chelate is a product of many parameters. The micelles of the side isomer are nearly twice the size than those of the corner isomer, and have much longer $\tau_{R}$ values. The slower tumbling for the micelles of the side isomer result in a higher relaxivity, despite the reduction of hydration that occurs from very fast water kinetics in the TSAP isomer.

\subsubsection{Biphenyl moiety interaction with poly- $\beta$-cyclodextrin}

The biphenyl groups can also be used to slow $\tau_{\mathrm{R}}$ through binding interactions with poly- $\beta$-CD. As a polymer of $10-11$ cyclodextrin units, poly- $\beta-C D$ is a large macromolecule with many more or less identical hydrophobic binding pockets to which the biphenyl moiety of GdBP-DOTA is able to bind noncovalently. The strength of the 


\section{CHAPTER 4}

interaction of each regioisomeric chelate with poly- $\beta-\mathrm{CD}$ was determined by relaxometric titration at concentrations below the CMC (Figure 4.17).

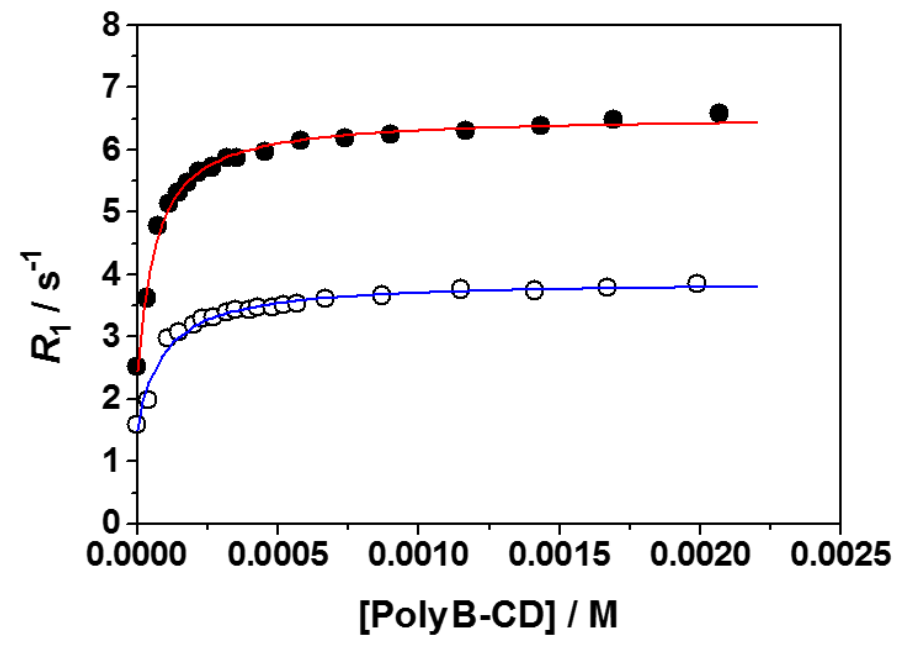

Figure 4.17: poly- $\beta-C D$ titration of corner (open, blue) and side (closed, red) isomers of GdBP-DOTA (0.15 mM and $0.20 \mathrm{mM}$, respectively) at $20 \mathrm{MHz}$ and $298 \mathrm{~K}$.

It is apparent from Figure 4.17 that the side isomer of GdBP-DOTA has both a higher relaxivity when bound and a higher binding affinity for poly- $\beta$-CD than the corner isomer. Equilibrium binding constants for the regioisomers of GdNB-DOTA can be calculated from the titration of poly- $\beta-\mathrm{CD}$. The result of this titration confirms that the side isomer more strongly binds poly- $\beta-\mathrm{CD}$ : the binding constant of the side isomer of GdBP-DOTA is $50 \%$ larger than that of the corner isomer (Table 4.5). The different orientation of the benzylic substituent on the regioisomers has been credited for influencing the binding constants with poly- $\beta-\mathrm{CD}$ in other benzylic substituted macrocyclic chelates. ${ }^{28}$ This is likely the cause of the different binding constants for the regioisomers of GdNB-DOTA as well. 
Table 4.5: Binding affinity constant of the GdBP-DOTA regioisomers to poly- $\beta-C D$ and relaxivity of the bound complex at $298 \mathrm{~K}$ and $20 \mathrm{MHz}$.

\begin{tabular}{ccc}
\hline Isomer & $K_{\mathrm{a}}\left(\mathrm{M}^{-1}\right)$ & $r_{1}^{\text {bound }}\left(\mathrm{mM}^{-1} \mathrm{~s}^{-1}\right)$ \\
\hline Corner & $(1.075 \pm 0.105) \times 10^{3}$ & $23.4 \pm 0.3$ \\
Side & $(1.580 \pm 0.092) \times 10^{3}$ & $30.8 \pm 0.2$ \\
\hline
\end{tabular}

The different binding constants of the GdBP-DOTA regioisomers have implications to the effectiveness of IB-DOTA as a BFC. The corner isomer predominates the chelation regioisomer distribution $2: 1$, but has a much lower binding affinity for poly- $\beta$-CD. If this result were to be reproduced in real bioconjugate systems then this could have a profound impact on the binding of the bioconjugate in vivo. ${ }^{1} \mathrm{H}$ NMRD profiles of the corner and side GdBP-DOTA isomers were collected (Figure 4.18) to probe how this difference in binding affinities affects relaxivity.
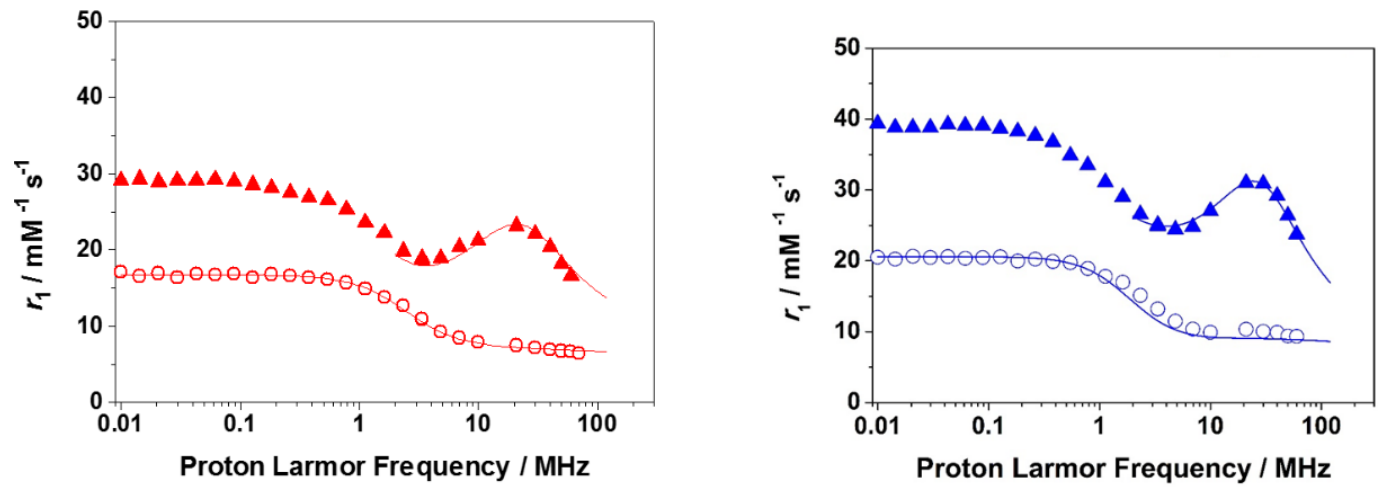

Figure 4.18: The NMRD profiles of free complex (open circles) and complex bound to poly- $\beta-C D$ (closed triangles) for the corner (red) and the side (blue) isomers of GdBP-DOTA at $298 \mathrm{~K}$ (at $0.15 \mathrm{mM}$ and at $0.20 \mathrm{mM}$, respectively). 
The ${ }^{1} \mathrm{H}$ NMRD profiles of the GdBP-DOTA chelates bound to poly- $\beta$-CD reflect those of slowly tumbling chelates with higher relaxivity at all fields and a characteristic hump at high fields. As in the micelle results, the side isomer displays higher relaxivity and a larger magnitude in the characteristic hump than the corner isomer. This reflects a stronger binding affinity, causing slower tumbling, in the side isomer of GdBP-DOTA. To further assess the differences in rotation of the regioisomers, the ${ }^{1} \mathrm{H}$ NMRD profiles of the free and bound regioisomers of GdBP-DOTA were fit to the SMB equations (Table 4.6). These fittings were completed using the $\tau_{\mathrm{M}}$ values from the GdNB-DOTA regioisomers.

Table 4.6: Parameters from fitting the ${ }^{1} \mathrm{H}$ NMRD profiles for the GdBP-DOTA corner and side isomers for the free chelates and bound to poly $-\beta-C D$.

a) fit using $a=4.0 \AA{ }^{298} D=2.24 \times 10^{-5} \mathrm{~cm}^{2} \mathrm{~s}^{-1}$ and $q=1$.

\begin{tabular}{ccccc}
\hline \multirow{2}{*}{ Parameter } & \multicolumn{2}{c}{ Free Complex } & \multicolumn{2}{c}{ Bound to poly- $\beta$-CD } \\
\cline { 2 - 5 } & Corner & Side & Corner & Side \\
\hline$\tau_{\mathrm{R}}(\mathrm{ps})$ global & 0.13 & 0.19 & 4.1 & 4.1 \\
$\tau_{\mathrm{R}}(\mathrm{ps})$ local & $/$ & $/$ & 0.38 & 0.55 \\
$\tau_{\mathrm{V}}(\mathrm{ps})$ & 18.6 & 17.4 & 26.7 & 13.1 \\
$\Delta^{2}\left(\times 10^{19} \mathrm{~s}^{-2}\right)$ & 1.10 & 1.08 & 0.36 & 0.37 \\
$S^{2}$ & $/$ & $/$ & 0.12 & 0.21 \\
\hline
\end{tabular}


The results of fitting these ${ }^{1} \mathrm{H}$ NMRD profiles indicates that as a free chelate the GdBP-DOTA corner and side regioisomers have similar $\tau_{\mathrm{R}}$ values, with the side isomer tumbling slightly slower - this agrees with what can be qualitatively seen in the ${ }^{1} \mathrm{H}$ NMRD profiles at high fields. The $\tau_{R}$ value for chelates bound to poly- $\beta-C D$ can be separated into global and local rotations. The global tumbling is defined by the motion of the large macromolecule in solution, and is the same for both regioisomers solutions. The differences in $\tau_{R}$ visible in the ${ }^{1} \mathrm{H}$ NMRD profiles of the bound regioisomers are a result of local rotation; the side isomer of GdBP-DOTA has much slower local rotation than the corner isomer. This difference in local rotation is a reflection of the binding constants for the regioisomers to poly- $\beta-\mathrm{CD}$ and perhaps is a result of the different orientations of the benzylic group relative to the macrocycle.

\subsubsection{Rotation of the benzylic substituent relative to the macrocycle}

The separation of global and local rotation has been proposed as an explanation for why bound BFC systems have not achieved the gains in relaxivity predicted by theory; faster local rotation means that the motion of the chelate is not effectively coupled to that of the macromolecule. ${ }^{119}$ In benzylic substituted macrocyclic chelates local rotation is most likely to occur through rotation around one of the bonds of the benzylic carbon. During analysis of the ${ }^{1} \mathrm{H}$ NMR data of the two regioisomeric chelates of EuNB-DOTA, we noticed that cross-peaks corresponding to exchange between the two benzylic protons of the same geometric isomer of a complex were not observed (Figure 4.19). Instead, ${ }^{1} \mathrm{H}-{ }^{1} \mathrm{H}$ EXSY peaks were observed between the benzylic protons of the SAP and TSAP isomers. 
A

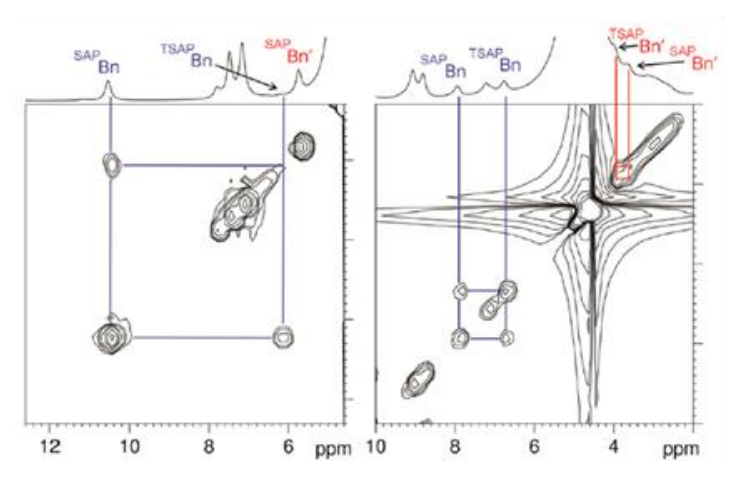

B

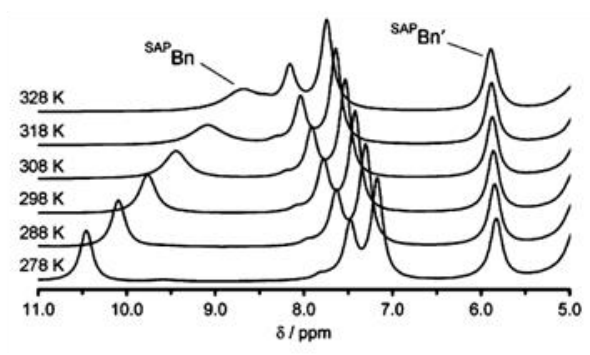

Figure 4.19: (A) The benzylic regions of the ${ }^{1} \mathrm{H}-{ }^{1} \mathrm{H}$ EXSY spectra of corner (left) and side (right) isomers of EuNB-DOTA $\left(\mathrm{D}_{2} \mathrm{O}, 400 \mathrm{MHz}, 283 \mathrm{~K}\right)$. (B) The benzylic and aromatic region of the variable temperature ${ }^{1} \mathrm{H}$ NMR spectra of the EuNB-DOTA corner isomer $\left(\mathrm{D}_{2} \mathrm{O}, 400 \mathrm{MHz}, 278-328 \mathrm{~K}\right.$ in $10 \mathrm{~K}$ increments).

The absence of a cross-peak in the EXSY spectrum is not evidence that exchange does not occur. To fully probe the exchange of the benzylic protons variable temperature ${ }^{1} \mathrm{H}$ NMR spectra were collected to observe exchange kinetics (Figure 4.19). In these experiments the $B n$ resonance of the SAP species shifts and becomes broad - suggesting it is in exchange with another proton. However, the $B n^{\prime}$ resonance of the same SAP species remains relatively sharp and un-shifted - suggesting that it is not in exchange with the SAP Bn resonance that is displaying coalescence behavior. Were the two benzylic protons of the SAP isomer readily in exchange, the coalescence behavior of the $B n$ resonance would also be reflected in $B n^{\prime}$ resonance. Because there is no evidence of exchange between the $B n$ and $B n^{\prime}$ positions, we may conclude that exchange of these benzylic protons is slow on the NMR time scale (slower than $10^{-3} \mathrm{~s}^{-1}$ ), this is more than five orders of magnitude slower than necessary to have a modulating effect on 
relaxivity. ${ }^{17,} 120$ This indicates that the rotation around the bond connecting the benzylic group to the macrocycle is slower than initially anticipated; however, it does not provide any information about rotation along the bond between the benzylic carbon and the most proximal aromatic carbon. This could suggest significantly less local rotation than attributed to these chelates bound to macromolecules or that the local rotation could primarily stem from the bond between the benzylic and aromatic carbons. These data may have implications for other chelates with benzylic substituted macrocycles. The possibility of slow local rotation in these complexes would indicate that there may be other factors impacting the achievement of maximum relaxivity that have been overlooked. One such factor is the influence of fast water exchange on the hydration of the chelates.

\subsection{Conclusions}

In efforts to understand how regioisomerism impacts LnNB-DOTA and its derivatives, commonly investigated as BFCs, we synthesized the $\mathrm{Eu}^{3+}, \mathrm{Gd}^{3+}$, and $\mathrm{Yb}^{3+}$ chelates of NB-DOTA and the biphenyl conjugates of the $\mathrm{Eu}^{3+}$ and $\mathrm{Gd}^{3+}$ complexes. As with the related LnNB-DOTMA chelates, regioisomerism of LnNB-DOTA was found to occur upon introduction of the $\mathrm{Ln}^{3+}$ ion into the chelate. From the crude chelation solutions we were able to purify corner and side regioisomers and assign the ${ }^{1} \mathrm{H}$ NMR spectra for the each isomer. These data indicate different distributions of the SAP and TSAP isomers for the regioisomers, resulting in faster water exchange kinetics for the side isomer. The experimentally determined $\tau_{M}$ values allowed the fitting of the ${ }^{1} \mathrm{H}$ NMRD profiles to reveal that the $\tau_{\mathrm{R}}$ value for the side isomer is longer than that of the corner isomer. 
Further studies into the tumbling of these chelates would confirm the relative tumbling rates of the regioisomers.

Previous investigations into conjugates of LnNB-DOTA for various bifunctional chelator applications do not consider the impact of regioisomerism that results from locking the helicity of the macrocycle. The differences in $\tau_{R}$ value for the LnNB-DOTA regioisomers, as well as the differences in the relative self-association and binding affinities of its biphenyl conjugates, impact the effectiveness of these chelates as targeted contrast agents. The two regioisomers appear to have different time-averaged orientations of the benzylic substituent relative to the macrocycle. This may be the origin of the slower tumbling of the side LnNB-DOTA isomer and may also be the source of the different properties seen in the biphenyl derivatives: the side GdBP-DOTA isomer also has a higher degree of self-association, forms micelles that are much larger than those of the corner isomer, and has higher binding affinities for poly- $\beta$-CD. These data indicate that the side isomer is a stronger candidate for BFC applications, but it is the minor species produced in the synthesis of the chelate.

In the process of assigning the proton resonances of the ${ }^{1} \mathrm{H}$ NMR spectra for EuNB-DOTA, we identified a dramatically slower rotation around the bond between the macrocycle and benzylic carbon than previously assumed. This has gone unnoticed in the published studies of the LnNB-DOTMA chelates. If this lack of free rotation around the macrocycle-benzylic bond corresponds to the local rotation occurring in this type of chelate, it negates the argument that local rotation limits the relaxivity of slowly tumbling chelates and opens space for considering how varying water exchange kinetics might 
influence hydration. Further investigations into the actual rates of local rotation are needed to indicate the influence this has on the efficacy of these agents. 


\section{CHAPTER 5: EXPERIMENTAL}

\section{General Remarks}

All solvents and reagents were purchased from commercial sources and used as received. Preparative RP-HPLC was completed on a Waters 2545 quaternary gradient module equipped with a Phenomenex Luna C18(2) 250x50 mm column at a flow rate of $50 \mathrm{~mL}$ $\min ^{-1}$ and continuous He sparge. Detection was by absorption, monitored at 270 (and 205) $\mathrm{nm}$ on a Waters $2489 \mathrm{UV} /$ visible detector. For all solvent systems $\mathrm{H}_{2} \mathrm{O}$ contained $0.037 \% \mathrm{HCl}$. Elution methods are varied by ligand/chelate as follows.

One and two dimensional NMR spectra were recorded on a Bruker Avance IIa or Avance III spectrometer (operating at $400.13 \mathrm{MHz}$ or $600.14 \mathrm{MHz}$, respectively) using a $5 \mathrm{~mm}$ broadband probe. The temperature was controlled on both instruments using the installed variable temperature controller model 2416 with BCU-05 chiller. Standard pulse sequences for ${ }^{1} \mathrm{H}, \mathrm{COSY}$, and EXSY analysis were utilized.

The fast field-cycling Stelar SMARtracer relaxometer (Stelar s.r.l., Mede, PV, Italy) equipped with a silver magnet was used for $1 / \mathrm{T}_{1}$ nuclear magnetic relaxation dispersion profiles of water protons measured over a continuum of magnetic field strength from 0.00024 to $0.25 \mathrm{~T}$ (corresponding to 0.01 to $10 \mathrm{~Hz}$ proton Larmor frequency). The relaxometer operates under complete computer control with an absolute uncertainty in the $1 / \mathrm{T}_{1}$ values of $\pm 1 \%$. The typical field sequences used were the NP (non-polarized) sequence between 10 and $4 \mathrm{MHz}$ and PP (pre-polarized) sequence between 4 and $0.01 \mathrm{MHz}$. Additional data at higher fields $(20-70 \mathrm{MHz})$ were measured on a Stelar 
Spinmaster relaxometer equipped with a Bruker WP80 magnet operating in the range 20 to $70 \mathrm{MHz}$.

Mass spectra were recorded on an electrospray (30000) LTQ-Orbitrap mass spectrometer (Thermo Scientific LTQ Orbitrap XL).

\subsection{Synthesis of thesis complexes}

$(1 R, 4 R, 7 R, 10 R)$-a, $a^{\prime}, a^{\prime \prime}, a^{\prime \prime \prime}$-tetramethyl-1,4,7,10-tetraazacyclododecane-1,4,7,10-

tetraaccetate lanthanide(III) complexes (LnDOTMA)

These chelates were prepared as previously reported and confirmed by ${ }^{1} \mathrm{H}$ NMR. ${ }^{63,93}$ Crystals were grown from aqueous mother liquors either by slow evaporation or by slow diffusion of EtOH. $\mathrm{Pr}^{3+}$ chelate ${ }^{1} \mathrm{H}$ NMR (400 MHz, $\mathrm{D}_{2} \mathrm{O}, 298 \mathrm{~K}$ ): $\delta(\mathrm{ppm}) 29.3(1 \mathrm{H}, \mathrm{s})$, $14.7(1 \mathrm{H}, \mathrm{s}) 6.7(3 \mathrm{H}, \mathrm{s}), 1.0(1 \mathrm{H}, \mathrm{s}),-1.7(1 \mathrm{H}, \mathrm{s}), 33.5(1 \mathrm{H}, \mathrm{s}) ; \mathrm{Eu}^{3+}$ chelate ${ }^{1} \mathrm{H}$ NMR (400 MHz, $\left.\mathrm{D}_{2} \mathrm{O}, 298 \mathrm{~K}\right): \delta(\mathrm{ppm}) 24.0(1 \mathrm{H}, \mathrm{s}), 2.3(3 \mathrm{H}, \mathrm{s}), 0.6(1 \mathrm{H}, \mathrm{s}), 0.05(1 \mathrm{H}, \mathrm{s}),-4.2$ $(1 \mathrm{H}, \mathrm{s}),-4.7(1 \mathrm{H}, \mathrm{s})$; Dy ${ }^{3+}$ chelate ${ }^{1} \mathrm{H}$ NMR $\left(400 \mathrm{MHz}, \mathrm{D}_{2} \mathrm{O}, 298 \mathrm{~K}\right)$ : shows proton signals indicative of chelation from 354 to $-556 \mathrm{ppm} ; \mathrm{Ho}^{3+}$ chelate ${ }^{1} \mathrm{H}$ NMR $(400 \mathrm{MHz}$, $\left.\mathrm{D}_{2} \mathrm{O}, 298 \mathrm{~K}\right): \delta(\mathrm{ppm}) 87.5(1 \mathrm{H}, \mathrm{s}), 40.3(3 \mathrm{H}, \mathrm{s})-35.5(1 \mathrm{H}, \mathrm{s}),-39.7(1 \mathrm{H}, \mathrm{s}),-58.5(1 \mathrm{H}, \mathrm{d}$, $\left.{ }^{3} J_{\mathrm{H}-\mathrm{H}}=-786 \mathrm{~Hz}\right),-200.6(1 \mathrm{H}, \mathrm{s}) ; \mathrm{Er}^{3+}$ chelate ${ }^{1} \mathrm{H}$ NMR $\left(400 \mathrm{MHz}, \mathrm{D}_{2} \mathrm{O}, 298 \mathrm{~K}\right): \delta(\mathrm{ppm})$ $37.5(1 \mathrm{H}, \mathrm{s}), 17.3(1 \mathrm{H}, \mathrm{s}),-32.2(1 \mathrm{H}, \mathrm{s}),-48.3(3 \mathrm{H}, \mathrm{s}),-112.3(1 \mathrm{H}, \mathrm{s}) ; \mathrm{Tm}^{3+}$ Chelate ${ }^{1} \mathrm{H}$ NMR (400 MHz, D $20,298 \mathrm{~K}): \delta(\mathrm{ppm}) 98.1(2 \mathrm{H}, \mathrm{s}), 73.8(2 \mathrm{H}, \mathrm{s})-101.6(3 \mathrm{H}, \mathrm{s}),-253.4$ $(1 \mathrm{H}, \mathrm{s})$ 
CHAPTER 5

$(1 R, 4 R, 7 R, 10 R) \quad$ and $\quad(1 S, 4 S, 7 S, 10 S)-a, a^{\prime}, a^{\prime \prime}, a^{\prime \prime \prime}$-tetramethyl [(S)-2-(4-nitrobenzyl)]1,4,7,10-tetraazacyclododecane-1,4,7,10-tetraaccetate lanthanide(III) complexes (LnNBDOTMA)

The ligands were synthesized and $\mathrm{Ln}^{3+}$ chelates made as previously described $\left(\mathrm{Gd}^{3+}: \mathrm{m} / z\right.$ (ESI-) 149.17715, $25 \%[\mathrm{M}]^{-} ; \mathrm{Eu}^{3+}: \mathrm{m} / z$ (ESI-) 744.17, $18 \%\left[\mathrm{M}^{-}\right)^{70,121,122}$ Isolation of the corner and side isomers was performed by reverse phase preparatory-HPLC as described by Table 5.1. $S$-RRRR chelates: corner $R_{\mathrm{T}}=17.1$ mins and side $R_{\mathrm{T}}=19.7$ mins; $S$-SSSS chelates: corner $R_{\mathrm{T}}=18.5$ mins and side $R_{\mathrm{T}}=21.6$ mins.

Table 5.1: Purification of LnNB-DOTMA chelates (from thesis of Ben Webber)

\begin{tabular}{ccccc}
\hline Time (min) & $0-3$ & $18-20$ & 20.1 & $21-26$ \\
\hline$\% \mathrm{H}_{2} \mathrm{O}(0.037 \% \mathrm{HCl})$ & 78 & 65 & 20 & 78 \\
$\% \mathrm{MeCN}$ & 22 & 35 & 80 & 22 \\
\hline
\end{tabular}

\section{(S)-2-(4-nitrobenzyl)-1,4,7,10-tetraazacyclododecane-1,4,7,10-tetraaccetate}

lanthanide(III) complexes (LnNB-DOTA)

The ligands were purchased as acid chlorides from Macrocyclics, and chelated with $\mathrm{EuCl}_{3}, \mathrm{YbCl}_{3}$, and $\mathrm{GdCl}_{3}$, as previously described in literature $\left(\mathrm{Gd}^{3+}: \mathrm{m} / \mathrm{z}\right.$ (ESI-) $693.11554,100 \%\left[\mathrm{M}^{-}\right) .^{72,113}$ Isolation of the corner and side isomers was performed by reverse phase preparatory-HPLC as described by Table 5.2. $\mathrm{Eu}^{3+}$ chelates: corner $\mathrm{R}_{\mathrm{T}}=$ 
9.4 mins and side $\mathrm{R}_{\mathrm{T}}=12.2$ mins; $\mathrm{Yb}^{3+}$ chelates: corner $\mathrm{R}_{\mathrm{T}}=9.8$ mins and side $\mathrm{R}_{\mathrm{T}}=$ 12.1 mins; $\mathrm{Gd}^{3+}$ chelates: corner $\mathrm{R}_{\mathrm{T}}=9.8$ mins and side $\mathrm{R}_{\mathrm{T}}=12.3$ mins. $\mathrm{Eu}^{3+}$ corner and side regioisomers $\left(400 \mathrm{MHz}, \mathrm{D}_{2} \mathrm{O}, 298 \mathrm{~K}\right)$ : show proton signals indicative of chelation from 40 to $-10 \mathrm{ppm}$ - complete assignment listed in Chapter $4 . \mathrm{Yb}^{3+}$ corner and side regioisomers (400 MHz, $\mathrm{D}_{2} \mathrm{O}, 298 \mathrm{~K}$ ): show proton signals indicative of chelation from 150 to $-100 \mathrm{ppm}$.

Table 5.2: Purification of LnNB-DOTA chelates

\begin{tabular}{ccccc}
\hline Time (min) & $0-3$ & $15-16$ & 16.1 & $17-21$ \\
\hline$\% \mathrm{H}_{2} \mathrm{O}(0.037 \% \mathrm{HCl})$ & 78 & 75 & 20 & 78 \\
$\% \mathrm{MeCN}$ & 22 & 25 & 80 & 22 \\
\hline
\end{tabular}

((S)-2-(4-(3-([1,1'-biphenyl]-4-ylmethyl)thioureido)benzyl)-1,4,7,10-

tetraazacyclododecane-1,4,7,10-tetraaccetate lanthanide(III) complexes (LnBP-DOTA)

The synthetic pathway to modify the nitro moiety to the biphenyl moiety is described in the literature; ${ }^{28}$ however, we purchased the thiocyanate ligand to allow for a single-step process. The thiocyanate ligand $(50 \mathrm{mg}$, FW 687.80) was dissolved in water $(25 \mathrm{~mL})$ then reacted with 4-phenylbenzylamine (19 $\mathrm{mg}, \mathrm{FW} 183.25)$ in dioxane $(15 \mathrm{~mL})$ at $\mathrm{pH} 6$ for $18 \mathrm{hrs}$ at room temperature. This synthesis is described in Scheme 4.1 and is based on the previously described synthesis of BP-DOTMA from NB-DOTMA. ${ }^{28,} 48$ Once the biphenyl moiety was established, these ligands were chelated with lanthanide chlorides, 
as in other LnDOTA-type systems. The corner and side isomers of these biphenyl chelates were purified by reverse phase preparatory-HPLC as described by Table 5.3. $\mathrm{Eu}^{3+}$ chelates $(\mathrm{m} / \mathrm{z}(\mathrm{ESI}-)) 883.2,24 \%\left[\mathrm{M}^{-}\right)$: corner $R_{\mathrm{T}}=14.4$ mins and side $R_{\mathrm{T}}=16.2$ mins; $\mathrm{Gd}^{3+}$ chelates $\left(\mathrm{m} / \mathrm{z}(\mathrm{ESI}-)\right.$ 888.2, $\left.100 \%[\mathrm{M}]^{-}\right)$: corner $R_{\mathrm{T}}=17.8$ mins and side $R_{\mathrm{T}}=$ 21.7 mins. $\mathrm{Eu}^{3+}$ corner and side regioisomers: (400 MHz, $\mathrm{D}_{2} \mathrm{O}, 298 \mathrm{~K}$ ): peaks present in the range of 35 to $-20 \mathrm{ppm}$ indicating chelation - regioisomer identification determined by the signal pattern of the most shifted side axial protons.

Table 5.3: Purification of LnBP-DOTA chelates

\begin{tabular}{cccccc}
\hline Time (min) & $0-3$ & $23-24$ & $24.1-25$ & $25.5-26.5$ & $27-32$ \\
\hline$\% \mathrm{H}_{2} \mathrm{O}(0.037 \% \mathrm{HCl})$ & 63 & 60 & 52.5 & 20 & 63 \\
$\% \mathrm{MeCN}$ & 37 & 40 & 47.5 & 80 & 37 \\
\hline
\end{tabular}




\section{REFERENCES}

\section{Complete list of references}

1. W. S. Hinshaw, P. A. Bottomley and G. N. Holland, Nature, 1977, 270, 722-723.

2. I. I. Rabi, Physical Review, 1937, 51, 652-654.

3. I. I. Rabi, J. R. Zacharias, S. Millman and P. Kusch, Physical Review, 1938, 53, 318318.

4. L. W. Alvarez and F. Bloch, Physical Review, 1940, 57, 111-122.

5. F. Bloch, W. W. Hansen and M. Packard, Physical Review, 1946, 70, 474-485.

6. F. Bloch, Physical Review, 1946, 70, 460-474.

7. E. M. Purcell, R. V. Pound and N. Bloembergen, Physical Review, 1946, 70, 986987.

8. E. M. Purcell, H. C. Torrey and R. V. Pound, Physical Review, 1946, 69, 37-38.

9. R. Damadian, Science, 1971, 171, 1151-1153.

10. D. Ware and P. Mansfield, Review of Scientific Instruments, 1966, 37, 1167-1171.

11. P. K. Grannell and P. Mansfield, Physics in Medicine and Biology, 1975, 20, 477.

12. P. C. Lauterbur, Nature, 1973, 242, 190-191.

13. E. R. Andrew, P. A. Bottomley, W. S. Hinshaw, G. N. Holland, W. S. Moore and C. Simaroj, Physics in Medicine and Biology, 1977, 22, 971.

14. J. Eisinger, R. G. Shulman and W. E. Blumberg, Nature, 1961, 192, 963-964.

15. M. Cohn and J. S. Leigh, Nature, 1962, 193, 1037-1040.

16. N. Bloembergen, E. M. Purcell and R. V. Pound, Physical Review, 1948, 73, 679712.

17. P. Caravan, J. J. Ellison, T. J. McMurry and R. B. Lauffer, Chemical Reviews, 1999, 99, 2293-2352.

18. é. Tóth, L. Helm and A. Merbach, in The Chemistry of Contrast Agents in Medical Magnetic Resonance Imaging, John Wiley \& Sons, Ltd, 2013, pp. 25-81.

19. J. Vipond, M. Woods, P. Zhao, G. Tircsó, J. Ren, S. G. Bott, D. Ogrin, G. E. Kiefer, Z. Kovacs and A. D. Sherry, Inorganic Chemistry, 2007, 46, 2584-2595.

20. M. Botta, European Journal of Inorganic Chemistry, 2000, 2000, 399-407.

21. Z. Kotková, G. A. Pereira, K. Djanashvili, J. Kotek, J. Rudovský, P. Hermann, L. Vander Elst, R. N. Muller, C. F. G. C. Geraldes, I. Lukeš and J. A. Peters, European Journal of Inorganic Chemistry, 2009, 2009, 119-136.

22. I. Solomon, Physical Review, 1955, 99, 559-565.

23. I. Solomon and N. Bloembergen, Journal of Chemical Physics, 1956, 25, 261-266.

24. N. Bloembergen, Journal of Chemical Physics, 1957, 27, 572-573.

25. L. O. Morgan and A. W. Nolle, The Journal of Chemical Physics, 1959, 31, 365368.

26. N. Bloembergen and L. O. Morgan, Journal of Chemical Physics, 1961, 34, 842850. 
27. S. Aime, A. S. Batsanov, M. Botta, R. S. Dickins, S. Faulkner, C. E. Foster, A. Harrison, J. A. K. Howard, J. M. Moloney, T. J. Norman, D. Parker, L. Royle and J. A. Gareth Williams, Journal of the Chemical Society, Dalton Transactions, 1997, 3623-3636.

28. S. Avedano, M. Botta, J. S. Haigh, D. L. Longo and M. Woods, Inorganic Chemistry, 2013, 52, 8436-8450.

29. D. H. Powell, A. E. Merbach, G. González, E. Brücher, K. Micskei, M. F. Ottaviani, K. Köhler, A. Von Zelewsky, O. Y. Grinberg and Y. S. Lebedev, Helvetica Chimica Acta, 1993, 76, 2129-2146.

30. C. F. Geraldes, R. D. Brown, 3rd, W. P. Cacheris, S. H. Koenig, A. D. Sherry and M. Spiller, Magn Reson Med, 1989, 9, 94-104.

31. A. Borel, J. F. Bean, R. B. Clarkson, L. Helm, L. Moriggi, A. D. Sherry and M. Woods, Chemistry - A European Journal, 2008, 14, 2658-2667.

32. A. D. Sherry, R. D. Brown, C. F. G. C. Geraldes, S. H. Koenig, K.-T. Kuan and M. Spiller, Inorganic Chemistry, 1989, 28, 620-622.

33. D. Parker, R. S. Dickins, H. Puschmann, C. Crossland and J. A. K. Howard, Chemical Reviews, 2002, 102, 1977-2010.

34. K. Micskei, D. H. Powell, L. Helm, E. Brücher and A. E. Merbach, Magnetic Resonance in Chemistry, 1993, 31, 1011-1020.

35. S. Aime, M. Botta, E. Terreno, P. L. Anelli and F. Uggeri, Magnetic Resonance in Medicine, 1993, 30, 583-591.

36. C. F. G. C. Geraldes, A. D. Sherry, I. Lázár, A. Miseta, P. Bogner, E. Berenyi, B. Sumegi, G. E. Kiefer, K. McMillan, F. Maton and R. N. Muller, Magnetic Resonance in Medicine, 1993, 30, 696-703.

37. F. Avecilla, Joop A. Peters and Carlos F. G. C. Geraldes, European Journal of Inorganic Chemistry, 2003, 2003, 4179-4186.

38. S. Aime, M. Botta, S. G. Crich, G. B. Giovenzana, R. Pagliarin, M. Piccinini, M. Sisti and E. Terreno, JBIC, J. Biol. Inorg. Chem., 1997, 2, 470-479.

39. S. Aime, A. Barge, A. S. Batsanov, M. Botta, D. D. Castelli, F. Fedeli, A. Mortillaro, D. Parker and H. Puschmann, Chemical Communications, 2002, 1120-1121.

40. D. Parker, H. Puschmann, A. S. Batsanov and K. Senanayake, Inorganic Chemistry, 2003, 42, 8646-8651.

41. S. Laus, R. Ruloff, É. Tóth and A. E. Merbach, Chemistry-A European Journal, 2003, 9, 3555-3566.

42. R. Ruloff, E. Toth, R. Scopelliti, R. Tripier, H. Handel and A. E. Merbach, Chemical Communications, 2002, 2630-2631.

43. A. Nonat, M. Giraud, C. Gateau, P. H. Fries, L. Helm and M. Mazzanti, Dalton Transactions, 2009, 8033-8046.

44. Z. Jaszberenyi, A. Sour, E. Toth, M. Benmelouka and A. E. Merbach, Dalton Transactions, 2005, 2713-2719.

45. T. Vitha, V. Kubicek, J. Kotek, P. Hermann, L. Vander Elst, R. N. Muller, I. Lukes and J. A. Peters, Dalton Transactions, 2009, 0, 3204-3214. 
46. S. Aime, A. S. Batsanov, M. Botta, J. A. K. Howard, D. Parker, K. Senanayake and G. Williams, Inorganic Chemistry, 1994, 33, 4696-4706.

47. X. Zhu, S. Chen, Q. Luo, C. Ye, M. Liu and X. Zhou, Chemical Communications, 2015, 51, 9085-9088.

48. B. C. Webber, C. Cassino, M. Botta and M. Woods, Inorganic Chemistry, 2015, 54, 2085-2087.

49. A. D. Sherry and Y. Wu, Current Opinion in Chemical Biology, 2013.

50. S. Aime, M. Botta and E. Terreno, in Advances in Inorganic Chemistry, Academic Press, 2005, vol. Volume 57, pp. 173-237.

51. R. N. Muller, B. Radüchel, S. Laurent, J. Platzek, C. Piérart, P. Mareski and L. Vander Elst, European Journal of Inorganic Chemistry, 1999, 1999, 1949-1955.

52. J. W. Chen, R. L. Belford and R. B. Clarkson, The Journal of Physical Chemistry A, 1998, 102, 2117-2130.

53. W. Huang, L. A. Tudorica, X. Li, S. B. Thakur, Y. Chen, E. A. Morris, I. J. Tagge, M. E. Korenblit, W. D. Rooney, J. A. Koutcher and J. Charles S. Springer, Radiology, 2011, 261, 394-403.

54. E. M. Stephens and C. M. Grisham, Biochemistry, 1979, 18, 4876-4885.

55. W. P. Cacheris, S. K. Nickle and A. D. Sherry, Inorganic Chemistry, 1987, 26, 958960.

56. J. F. Desreux, Inorganic Chemistry, 1980, 19, 1319-1324.

57. H. Stetter and W. Frank, Angewandte Chemie International Edition in English, 1976, 15, 686-686.

58. M. R. Spirlet, J. Rebizant, J. F. Desreux and M. F. Loncin, Inorganic Chemistry, 1984, 23, 359-363.

59. H. G. Brittain and J. F. Desreux, Inorganic Chemistry, 1984, 23, 4459-4466.

60. M. Meyer, V. Dahaoui-Gindrey, C. Lecomte and R. Guilard, Coordination Chemistry Reviews, 1998, 178-180, Part 2, 1313-1405.

61. S. Aime, M. Botta and G. Ermondi, Inorganic Chemistry, 1992, 31, 4291-4299.

62. S. Aime, M. Botta, M. Fasano, M. P. M. Marques, C. F. G. C. Geraldes, D. Pubanz and A. E. Merbach, Inorganic Chemistry, 1997, 36, 2059-2068.

63. S. Aime, M. Botta, Z. n. Garda, B. E. Kucera, G. Tircso, V. G. Young and M. Woods, Inorganic Chemistry, 2011, 50, 7955-7965.

64. B. C. Webber and M. Woods, Dalton Transactions, 2014, 43, 251-258.

65. A. Beeby, I. M. Clarkson, R. S. Dickins, S. Faulkner, D. Parker, L. Royle, A. S. de Sousa, J. A. Gareth Williams and M. Woods, Journal of the Chemical Society, Perkin Transactions 2, 1999, 493-504.

66. S. Hoeft and K. Roth, Chemische Berichte, 1993, 126, 869-873.

67. B. C. Webber and M. Woods, Inorganic Chemistry, 2012, 51, 8576-8582.

68. G. Tircso, B. C. Webber, B. E. Kucera, V. G. Young and M. Woods, Inorganic Chemistry, 2011, 50, 7966-7979.

69. M. Woods, M. Botta, S. Avedano, J. Wang and A. D. Sherry, Dalton Transactions, 2005, 0, 3829-3837. 
70. M. Woods, Z. Kovacs, S. Zhang and A. D. Sherry, Angewandte Chemie International Edition, 2003, 42, 5889-5892.

71. R. S. Ranganathan, N. Raju, H. Fan, X. Zhang, M. F. Tweedle, J. F. Desreux and V. Jacques, Inorganic Chemistry, 2002, 41, 6856-6866.

72. M. Woods, Z. Kovacs, R. Kiraly, E. Brücher, S. Zhang and A. D. Sherry, Inorganic Chemistry, 2004, 43, 2845-2851.

73. J. A. K. Howard, A. M. Kenwright, J. M. Moloney, D. Parker, M. Woods, M. Port, M. Navet and O. Rousseau, Chem. Commun. (Cambridge), 1998, 1381-1382.

74. M. Woods, S. Aime, M. Botta, J. A. K. Howard, J. M. Moloney, M. Navet, D. Parker, M. Port and O. Rousseaux, Journal of the American Chemical Society, 2000, 122, 9781-9792.

75. L. Di Bari, G. Pintacuda and P. Salvadori, European Journal of Inorganic Chemistry, 2000, 2000, 75-82.

76. R. S. Ranganathan, R. K. Pillai, N. Raju, H. Fan, H. Nguyen, M. F. Tweedle, J. F. Desreux and V. Jacques, Inorganic Chemistry, 2002, 41, 6846-6855.

77. A. Bianchi, L. Calabi, F. Corana, S. Fontana, P. Losi, A. Maiocchi, L. Paleari and B. Valtancoli, Coordination Chemistry Reviews, 2000, 204, 309-393.

78. D. Parker, Coordination Chemistry Reviews, 2000, 205, 109-130.

79. S. K. Hekmatyar, P. Hopewell, S. K. Pakin, A. Babsky and N. Bansal, Magnetic Resonance in Medicine, 2005, 53, 294-303.

80. L. Helm and A. E. Merbach, Coordination Chemistry Reviews, 1999, 187, 151-181.

81. S. H. Koenig and M. Epstein, Journal of Chemical Physics, 1975, 63.

82. J. Kotek, J. Rudovský, P. Hermann and I. Lukeš, Inorganic Chemistry, 2006, 45, 3097-3102.

83. A. Barge, M. Botta, D. Parker and H. Puschmann, Chemical Communications, 2003, 1386-1387.

84. S. Aime, A. Barge, M. Botta, J. A. K. Howard, R. Kataky, M. P. Lowe, J. M. Moloney, D. Parker and A. S. de Sousa, Chemical Communications, 1999, 10471048.

85. J. Rohovec, M. Kývala, P. Vojtíšek, P. Hermann and I. Lukeš, European Journal of Inorganic Chemistry, 2000, 2000, 195-203.

86. J. Rohovec, P. Vojtisek, P. Hermann, J. Ludvik and I. Lukes, Journal of the Chemical Society, Dalton Transactions, 2000, 141-148.

87. R. L. Luck, C. L. Maupin, D. Parker, J. P. Riehl and J. A. Gareth Williams, Inorganica Chimica Acta, 2001, 317, 331-337.

88. J. Rohovec, P. Vojtisek, P. Hermann, J. Mosinger, Z. Zak and I. Lukes, Journal of the Chemical Society, Dalton Transactions, 1999, 3585-3592.

89. J. Klimentová and P. Vojtı'šek, Journal of Molecular Structure, 2007, 826, 82-88.

90. F. Benetollo, G. Bombieri, L. Calabi, S. Aime and M. Botta, Inorganic Chemistry, 2003, 42, 148-157.

91. L. Di Bari, G. Pintacuda and P. Salvadori, Journal of the American Chemical Society, 2000, 122, 5557-5562. 
92. D.-F. Chen and T.-Z. Jin, Gaodeng Xuexiao Huaxue Xuebao, 1996, 17, 1179-1181.

93. K. M. Payne, E. J. Valente, S. Aime, M. Botta and M. Woods, Chemical Communications, 2013, 49, 2320-2322.

94. D. J. Richens, Wiley, 1997, Chichester.

95. B. C. Webber and M. Woods, Nature Chem, 2012, Submitted.

96. S. Aime, A. Barge, M. Botta, A. S. De Sousa and D. Parker, Angewandte Chemie International Edition, 1998, 37, 2673-2675.

97. S. Aime, M. Botta, D. Parker and J. A. G. Williams, Journal of the Chemical Society, Dalton Transactions, 1996, 0, 17-23.

98. C. Cossy, L. Helm and A. E. Merbach, Inorganica Chimica Acta, 1987, 139, 147149.

99. C. Cossy, L. Helm and A. E. Merbach, Inorganic Chemistry, 1988, 27, 1973-1979.

100. D. Pubanz, G. Gonzalez, D. H. Powell and A. E. Merbach, Inorganic Chemistry, 1995, 34, 4447-4453.

101. F. A. Dunand, S. Aime and A. E. Merbach, Journal of the American Chemical Society, 2000, 122, 1506-1512.

102. S. Aime, A. Barge, M. Botta, D. Parker and A. S. De Sousa, Journal of the American Chemical Society, 1997, 119, 4767-4768.

103. S. Aime, A. Barge, J. I. Bruce, M. Botta, J. A. K. Howard, J. M. Moloney, D. Parker, A. S. de Sousa and M. Woods, Journal of the American Chemical Society, 1999, 121, 5762-5771.

104. T. J. Swift and R. E. Connick, The Journal of Chemical Physics, 1962, 37, 307-320.

105. T. J. Swift and R. E. Connick, The Journal of Chemical Physics, 1964, 41, 25532554.

106. S. Aime, M. Botta, M. Fasano, S. Paoletti and E. Terreno, Chemistry - A European Journal, 1997, 3, 1499-1504.

107. M. R. Churchill, Inorganic Chemistry, 1973, 12, 1213-1214.

108. S. M. Cohen, J. Xu, E. Radkov, K. N. Raymond, M. Botta, A. Barge and S. Aime, Inorganic Chemistry, 2000, 39, 5747-5756.

109. R. B. Clarkson, J. H. Hwang and R. L. Belford, Magnetic Resonance in Medicine, 1993, 29, 521-527.

110. M. B. Yim and M. W. Makinen, Journal of Magnetic Resonance (1969), 1986, 70, 89-105.

111. P. Caravan, A. V. Astashkin and A. M. Raitsimring, Inorganic Chemistry, 2003, 42, 3972-3974.

112. M. K. Moi, C. F. Meares and S. J. DeNardo, Journal of the American Chemical Society, 1988, 110, 6266-6267.

113. K. M. Payne and M. Woods, Bioconjugate Chemistry, 2015, 26, 338-344.

114. D. Delli Castelli, M. C. Caligara, M. Botta, E. Terreno and S. Aime, Inorganic Chemistry, 2013, 52, 7130-7138. 
115. D. H. Powell, O. M. N. Dhubhghaill, D. Pubanz, L. Helm, Y. S. Lebedev, W. Schlaepfer and A. E. Merbach, Journal of the American Chemical Society, 1996, 118, 9333-9346.

116. G. Lipari and A. Szabo, Journal of the American Chemical Society, 1982, 104, 4546-4559.

117. G. Lipari and A. Szabo, Journal of the American Chemical Society, 1982, 104, 4559-4570.

118. E. Ruckenstein and R. Nagarajan, The Journal of Physical Chemistry, 1975, 79, 2622-2626.

119. P. Caravan, M. T. Greenfield, X. Li and A. D. Sherry, Inorganic Chemistry, 2001, 40, 6580-6587.

120. S. Aime, M. Botta, M. Fasano and E. Terreno, Chem. Soc. Rev., 1998, 27, 19-29.

121. O. Renn and C. F. Meares, Bioconjugate Chemistry, 1992, 3, 563-569.

122. T. J. McMurry, M. Brechbiel, K. Kumar and O. A. Gansow, Bioconjugate Chemistry, 1992, 3, 108-117.

123. S. Aime, L. Calabi, C. Cavallotti, E. Gianolio, G. B. Giovenzana, P. Losi, A. Maiocchi, G. Palmisano and M. Sisti, Inorganic Chemistry, 2004, 43, 7588-7590.

124. S. Aime, G. Bombieri, C. Cavallotti, G. B. Giovenzana, D. Imperio and N. Marchini, Inorganica Chimica Acta, 2008, 361, 1534-1541. 


\section{APPENDIX: OTHER EXPERIMENTAL WORK NOT COMPLETED AS PART OF THIS DISSERTATION}

\section{A.1 Developing AAZTA for potential modification}

\section{General Remarks}

The synthesis of the heptadentate ligand 6-amino-6-methylperhydro-1,4diazepinetetraacetic acid (AAZTA) is outlined in literature ${ }^{123,124}$ and was followed closely in the attempt to synthesize an amide analog: 1,4-bis(benzyl acetaamino)-6-bis(benzyl acetaamino)-6-methylperhydro-1,4-diazepine (AAZTA-tetraamide).

\section{AAZTA-1 (7 membered ring formation)}

In ethanol, 1 equivalent N,N'-dibenzylethylenediamine (FW 240), two equivalents acetic acid (FW 60), and one equivalent of nitroethane (FW 75) were dissolved. To this solution, excess paraformaldehyde was added. The solution was heated to reflux for $3.5 \mathrm{hrs}$. The solvent was removed in vacuo, leaving the seven-membered ring with a nitro group and two phenyl protecting groups (1,4-dibenzyl-6-methyl-6-nitroperhydro-1,4diazepine, FW 339). $400 \mathrm{MHz}, \mathrm{D}_{2} \mathrm{O}, 298 \mathrm{~K}: \delta(\mathrm{ppm}) 7.3(10 \mathrm{H}, \mathrm{m}, \mathrm{Ar}), 3.8(2 \mathrm{H}, \mathrm{d}$, $\left.{ }^{3} J_{\mathrm{H}-\mathrm{H}}=12.7 \mathrm{~Hz}, \mathrm{CH}_{2}\right), 3.6\left(4 \mathrm{H}, \mathrm{dd},{ }^{2} J_{\mathrm{H}-\mathrm{H}}=20.9,{ }^{3} J_{\mathrm{H}-\mathrm{H}}=5.5, \mathrm{Bn}\right.$ and $\mathrm{Bn}$ '), $2.9(2 \mathrm{H}, \mathrm{d}$, $\left.{ }^{3} J_{\mathrm{H}-\mathrm{H}}=13.6 \mathrm{~Hz}, \mathrm{CH}_{2}\right), 2.6\left(4 \mathrm{H}, \mathrm{m}, \mathrm{NC}_{2} \underline{\mathrm{CH}}_{2} \mathrm{~N}\right), 1.3\left(3 \mathrm{H}, \mathrm{s}, \mathrm{CH}_{3}\right)$

AAZTA-2 (reductive amination of nitro group and removal of protecting groups)

AAZTA-1 was dissolved in ethanol with catalytic amounts of $10 \% \mathrm{Pd} / \mathrm{C}$ and hydrogenated at 60 psi for 10 days. The $\mathrm{Pd} / \mathrm{C}$ was filtered from solution, and solvent was 
removed in vacuo. This resulted in the seven-membered ring with three amines available for reaction with pendent arms (6-amino-6-mehtylperhydro-1,4-diazepine). $\mathrm{m} / \mathrm{z}$ ESI+ $130.13,100 \%[\mathrm{M}]^{+}$

AAZTA- 3 (alkylation of ring)

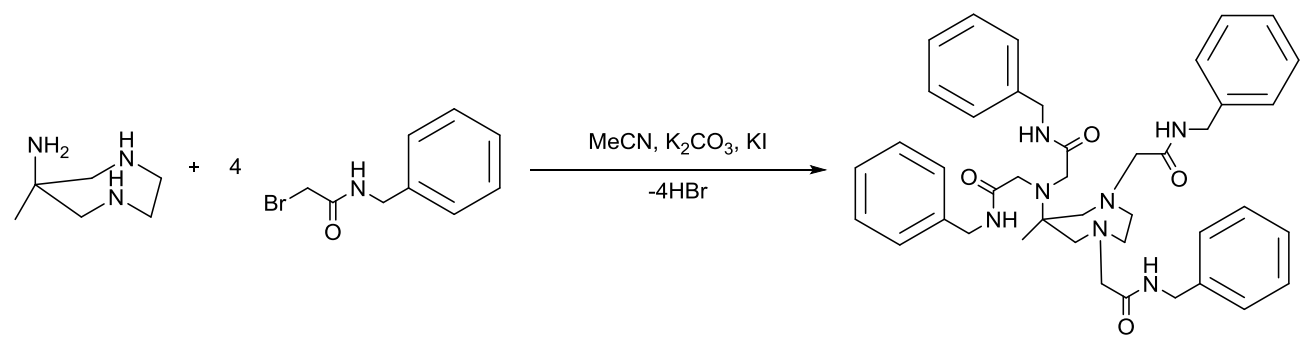

AAZTA-2 was dissolved in acetonitrile, with approximately seven equivalents potassium carbonate and catalytic amounts of potassium iodide, was cooled in an ice bath. Benzyl bromoacetaamide was dissolved in acetonitrile and slowly added piecewise. The solution was allowed to warm to room temperature, and then heated to reflux over two days. The solvent was removed in vacuo, and the salts removed by separation with basic $\mathrm{H}_{2} \mathrm{O}$ and dichloromethane. These steps produced a small amount of tetra-alkylated ring, so the process was repeated (twice), adding similar amounts of $\mathrm{K}_{2} \mathrm{CO}_{3}$ and $\mathrm{KI}$, with a small amount more benzyl bromoacetaamide for refluxing an additional two days. This resulted in the impure AAZTA-3, with protecting benzyl groups on the amides. $\mathrm{m} / \mathrm{z}$ ESI+ 571.33 and $718.41,35$ and $21 \%[\mathrm{M}]^{+}$, for tri and tetra products, respectively. Purification of half of the AAZTA-3 product was attempted by silica gel chromatography: $8 \% \mathrm{MeOH}$ in DCM. Results show that no product was collected from this column - it is suspected that the product was lost on the solvent front. Purification of the second half of the product was again attempted by silica gel chromatography: $2 \% \mathrm{MeOH}$ in $\mathrm{DCM} ; 80 \% \mathrm{MeOH}$ in 
DCM; $5 \% \mathrm{NH}_{4} \mathrm{OH}$ in $\mathrm{H}_{2} \mathrm{O}$. None of the collected fractions (including the solvent front) showed tetra-alkylated product by MS analysis - the conclusion is that this product did not come off the silica gel. Other attempts at purification by crystallization included combinations of ethanol and $\mathrm{H}_{2} \mathrm{O}$ as well as methanol and $\mathrm{H}_{2} \mathrm{O}$ : all attempts resulted in fine amorphous solids.

\section{A.2 Developing NB-DO3MA ligands for synthesizing NB-DOTMA-type analogs with differing donor atoms, specifically at the axial donor position}

\section{General Remarks}

The synthesis of the (4R,7R,10R)- and (4S,7S,10S)-a, a',a",-trimethyl [(S)-2-(4nitrobenzyl)]-1,4,7,10-tetraazacyclododecane-4,7,10-triaccetic acid ligand follows, closely, the synthesis of NB-DOTMA, but results from a 3:1 ratio of the pendent arm to nitrobenzyl cyclen during the alkylation process. Note: Some of the syntheses for NB-DO3MA and NB-DOTMA ligands also resulted in NB-DO2MA ligands, as detected by the mass spectrometer.

LnNB-DO3MA

NB-DO3MA was prepared according to the literature for NB-DOTMA, with an altered ratio of pendent arm to nitrobenzyl cyclen $\left(\mathrm{m} / \mathrm{z}\right.$ ESI+ 566.31, $\left.100 \%[\mathrm{M}]^{+}\right)$, and purified by silica gel chromatography: $100 \%$ DCM $(0.5 \mathrm{~L}) ; 5 \% \mathrm{MeOH}$ in DCM $(0.6 \mathrm{~L})$; $20 \% \mathrm{MeOH}$ in DCM $(0.6 \mathrm{~L}) ; 5 \% \mathrm{NH}_{4} \mathrm{OH}$ in $\mathrm{MeOH}(0.5 \mathrm{~L})$. Methyl esters were removed with $\mathrm{LiOH}$ in aqueous solution. Chelation with lanthanide chlorides were preformed similarly as with NB-DOTMA. Purification by reverse phase preparative- 
HPLC of $\mathrm{Eu}^{3+}$ chelates of NB-DO3MA, as described by Table A.1. Eu ${ }^{3+} S-R R R$ and $S$ SSS crude chelation products $\left(400 \mathrm{MHz}, \mathrm{D}_{2} \mathrm{O}, 298 \mathrm{~K}\right.$ ): show proton signals indicative of chelation from 25 to $-22 \mathrm{ppm}$.

Table A.1: Purification attempt of LnNB-DO3MA chelates

\begin{tabular}{cccccc}
\hline Time $(\min )$ & $0-3$ & 8 & 18 & $19-21$ & $22-27$ \\
\hline$\% \mathrm{H}_{2} \mathrm{O}(0.037 \% \mathrm{HCl})$ & 80 & 58 & 43 & 20 & 80 \\
$\% \mathrm{MeCN}$ & 20 & 42 & 57 & 80 & 20 \\
\hline
\end{tabular}

NB-DO3MAPY

The free base of purchased bromo-methyl pyridine $(0.50 \mathrm{~g})$ was recovered from aqueous solution $(\mathrm{pH}=13)$ by extraction with DCM $(4 \times 50 \mathrm{~mL})$ and confirmed by ${ }^{1} \mathrm{H}$ NMR. The alkylation of the free base (0.05 g, FW 172) with the $S$-RRR NB-DO3MA-methyl ester ligand (0.043 g, FW 523.6) occurred in $\mathrm{MeCN}(60 \mathrm{~mL})$ with $\mathrm{K}_{2} \mathrm{CO}_{3}(0.478 \mathrm{~g}$, FW 138.2) at reflux for one weeks. Product was extracted from reaction solution with $\mathrm{pH}=11.5$ $\mathrm{H}_{2} \mathrm{O}(20 \mathrm{~mL})$ with DCM $(3 \times 40 \mathrm{~mL})$; solvent removed in vacuo; ${ }^{1} \mathrm{H}$ NMR and MS $(\mathrm{m} / \mathrm{z}$ ESI+ 652.36, $\left.82 \%[\mathrm{M}]^{+}\right)$confirm impure product $(77 \mathrm{mg})$. Attempt at purification by silica gel column was not successful: 100\% DCM (0.6 L), 10\% MeOH in DCM (0.9 L), $20 \% \mathrm{MeOH}$ in DCM (1.0 L), $80 \% \mathrm{MeOH}$ in DCM $(1.0 \mathrm{~L}), 5 \% \mathrm{NH}_{4} \mathrm{OH}$ in $\mathrm{MeOH}$ $(0.5 \mathrm{~L})$. The methyl ester groups were removed from unpurified product using $\mathrm{LiOH}$ $(0.26 \mathrm{~g})$ dissolved in $\mathrm{H}_{2} \mathrm{O}(10 \mathrm{~mL})$; the product was dissolved in $\mathrm{MeCN}(1 \mathrm{~mL})$ and 
mixed with THF ( $10 \mathrm{~mL}$; the mixture was heated and stirred vigorously for $18 \mathrm{hrs}$. This acid ligand product was split into three vials: 1 for later purification, 1 for $\mathrm{Gd}^{3+}$ chelates, and 1 for $\mathrm{Eu}^{3+}$ chelates. For the $\mathrm{Ln}^{3+}$ chelates, the chloride salts $\left(0.064 \mathrm{~g} \mathrm{EuCl}_{3} ; 0.098\right.$ $\mathrm{GdCl}_{3}$ ) were added to their respective vials, the aqueous solutions were maintained at $\mathrm{pH}=5$, at $60{ }^{\circ} \mathrm{C}$ for one week. The impure $\mathrm{Eu}^{3+}$ chelate was confirmed by ${ }^{1} \mathrm{H}$ NMR (400 MHz, $\mathrm{D}_{2} \mathrm{O}, 298 \mathrm{~K}$ : small broad peaks $\delta$ (ppm) 36.3, 35.6, 29.8, 15.7, -5.2, -5.8, -6.1, $-6.9,-8.4,-9.0,-12.4,-13.8,-16.6,-17.5,-21.0,-21.9$ indicating chelate formation). The impure $\mathrm{Eu}^{3+} S-R R R$ chelate was confirmed by MS $\left(\mathrm{m} / z \mathrm{ESI}+765.21,67 \%[\mathrm{M}]^{+}\right)$and ${ }^{1} \mathrm{H}$ NMR peaks between 40 and $-25 \mathrm{ppm}$. Chelates were purified by reverse phase preparative-HPLC, as described in Table A.2, here was little chelate product in the crude material, and it was lost in this process.

Table A.2: Purification attempt of LnNB-DO3MAPy chelates

\begin{tabular}{cccccc}
\hline Time (min) & $0-3$ & 8 & 10 & $13-18$ & $19-24$ \\
\hline$\% \mathrm{H}_{2} \mathrm{O}(0.037 \% \mathrm{HCl})$ & 90 & 80 & 75 & 20 & 90 \\
$\% \mathrm{MeCN}$ & 10 & 20 & 25 & 80 & 10 \\
\hline
\end{tabular}


PyCOOH arm
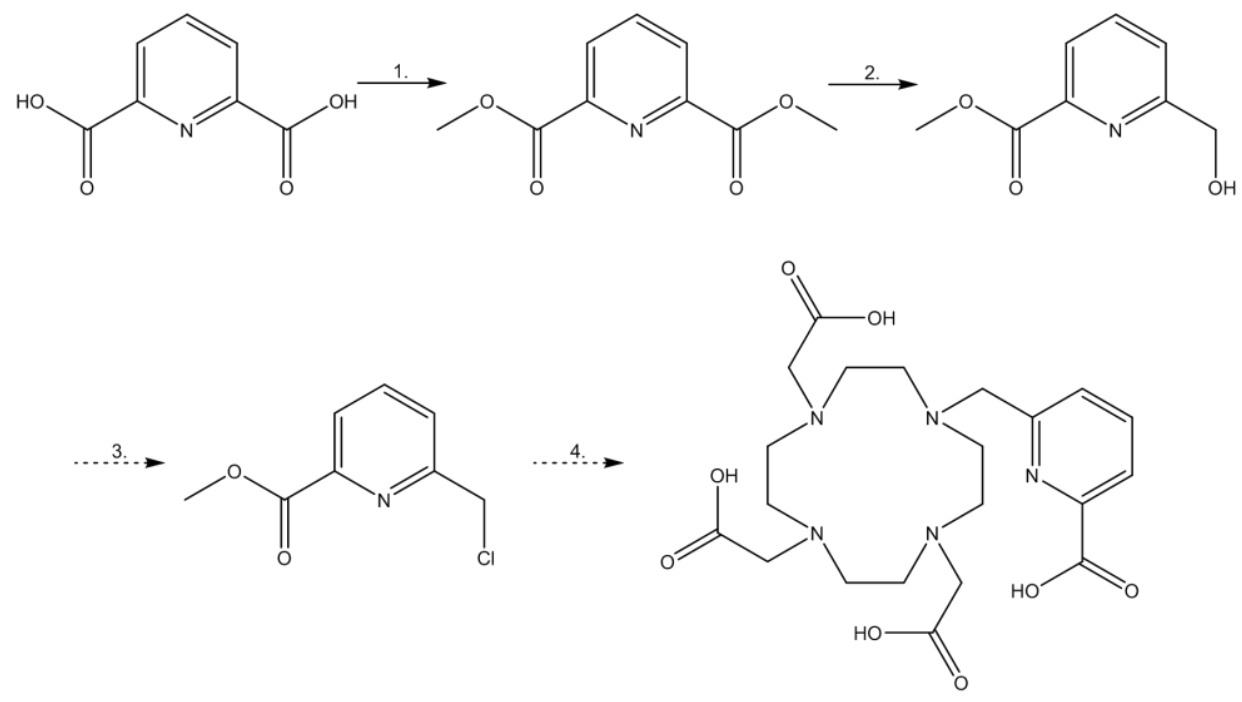

(1.) Dipicolinic acid (3.98 g), suspended in $\mathrm{MeOH}(50 \mathrm{~mL})$ and conc. $\mathrm{H}_{2} \mathrm{SO}_{4}(10 \mathrm{~mL})$, was heated at $80{ }^{\circ} \mathrm{C}$ for $18 \mathrm{hrs}$. The $\mathrm{pH}$ was raised to $\mathrm{pH}=9$ and product was extracted with DCM four times; solvent removed in vacuo. This produced relatively pure (by ${ }^{1} \mathrm{H}$ NMR) dimethyl ester product (approx. 3.0 g). $400 \mathrm{MHz}, \mathrm{CDCl}_{3}, 298 \mathrm{~K}: \delta(\mathrm{ppm}) 8.2(2 \mathrm{H}$, d, $\left.{ }^{3} J_{\mathrm{H}-\mathrm{H}}=7.8, m-\mathrm{Ar}\right), 7.9\left(1 \mathrm{H}, \mathrm{dd},{ }^{3} J_{\mathrm{H}-\mathrm{H}}=7.8, p-\mathrm{Ar}\right), 6.2\left(6 \mathrm{H}, \mathrm{s}, \mathrm{CH}_{3}\right)$. (2. - failed) Half of the dimethyl ester was suspended in $\mathrm{MeOH}$ at $0{ }^{\circ} \mathrm{C}$ and reacted with $\mathrm{NaBH}_{4}$ (approx. $1.0 \mathrm{~g}$ ) for $2.5 \mathrm{hrs}$. This reaction was quenched with $\mathrm{NaHCO}_{3}(19 \mathrm{~g})$ dissolved in $\mathrm{H}_{2} \mathrm{O}(200 \mathrm{~mL})$ and the $\mathrm{MeOH}$ was removed in vacuo. The aqueous solution $(\mathrm{pH}=11)$ was extracted with DCM three times. The organic layer showed no white solids after solvent removed; after $18 \mathrm{hrs}$ crystals formed from the aqueous layer. ${ }^{1} \mathrm{H}$ NMR of the crystals suggested the presence of some product - these were dissolved in $\mathrm{H}_{2} \mathrm{O}(\mathrm{pH}=7)$ and extracted three times with DCM - no product was found in the organic layer by ${ }^{1} \mathrm{H}$ NMR. (2. - failed) With the second half of the dimethyl ester product hydrolysis was 
attempted with $\mathrm{NaOH}$ at $60{ }^{\circ} \mathrm{C}$ in 1:1 v/v THF/ $\mathrm{H}_{2} \mathrm{O}: \mathrm{NaOH}$ solution and THF were added to maintain $\mathrm{pH}=13$ and soluble products - no product was found by ${ }^{1} \mathrm{H}$ NMR after 18 hrs or 3 days.

2-bromomethyl-6-methylphthalamide pyridine (PyAm arm)

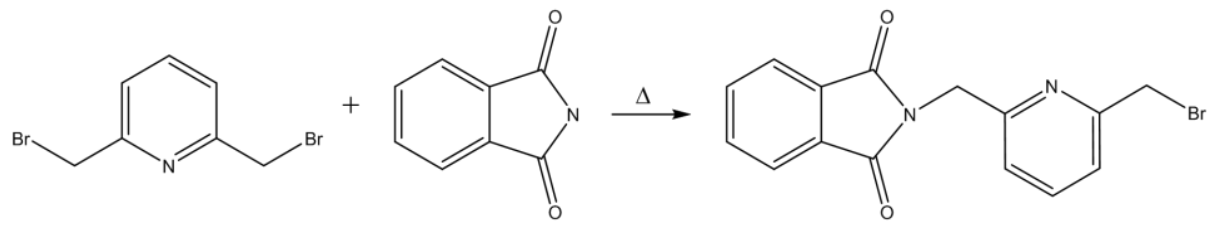

2,6-di(bromomethyl)pyridine and phthalamide were reacted at reflux for 24 hrs. TLC analysis suggested product formation. Purification was attempted by silica gel chromatography: $100 \%$ DCM (eluted the bromo starting material); $20 \% \mathrm{MeOH}$ in DCM (eluted the product and phthalamide). The product was extracted from the starting materials with an acid wash $(\mathrm{pH}=1)$ followed by a base wash $(\mathrm{pH}=12)$. Product was found in the reduced organic phase from the acid wash. Continued acid and base washing did not further purify the product; instead the product was purified by precipitating from a solution of DCM with hexanes. $400 \mathrm{MHz}, \mathrm{CDCl}_{3}, 298 \mathrm{~K}: \delta(\mathrm{ppm}) 7.9(2 \mathrm{H}, \mathrm{m}), 7.7$ $(2 \mathrm{H}, \mathrm{m}), 7.6\left(1 \mathrm{H}, \mathrm{dd},{ }^{3} J_{\mathrm{H}-\mathrm{H}}=7.6\right), 7.3\left(1 \mathrm{H}, \mathrm{d},{ }^{3} J_{\mathrm{H}-\mathrm{H}}=7.6\right), 7.1\left(1 \mathrm{H}, \mathrm{d},{ }^{3} J_{\mathrm{H}-\mathrm{H}}=7.6\right), 5.0$ $(2 \mathrm{H}, \mathrm{s}), 4.4(2 \mathrm{H}, \mathrm{s})$.

DO3APyAm, protected

2-bromomethyl-6-methylphthalamide pyridine $(0.237 \mathrm{~g})$ dissolved in DCM $(15 \mathrm{~mL})$ was used in an alkylation reaction (100 $\mathrm{mL} \mathrm{MeCN}$ with $\left.0.10 \mathrm{~g} \mathrm{~K}_{2} \mathrm{CO}_{3}\right)$ with DO3A-t-butyl ester ligand $(0.2953 \mathrm{~g})$, heated to $70{ }^{\circ} \mathrm{C}$ for $18 \mathrm{hrs}$. The organic product was separated 
from water $(\mathrm{pH}=6.5)$ into DCM, and the solvent was removed under pressure. $\mathrm{m} / \mathrm{z}$ $(\mathrm{ESI}+)$ 765.45, $100 \%[\mathrm{M}]^{+}$

10-(2-methyl-4-aminomethyl-pyridine)-1,4,7,10-tetraazacyclododecane-4,7,10-triaccetic acid (DO3A-PyAm, unprotected)

Removal of phthalamide protecting group attempted on two occasions with 1.2 equivalence of hydrazine monohydrate in ethanol, but failed to remove all the phthalamide. Removal of the $t$-butyl esters using $2 \mathrm{M} \mathrm{HCl}(10 \mathrm{~mL}$, in EtOH $)$ at room temperature for $18 \mathrm{hrs}$ resulted in removal of the remaining phthalamide protecting group as well as the $t$-butyl groups. $m / z(\mathrm{ESI}+) 467.33,68 \%[\mathrm{M}]^{+} ; \mathrm{m} / z$ (ESI-) 465.33, $37 \%[\mathrm{M}]^{-}$

\section{LnDO3APyAm}

The $\mathrm{Eu}^{3+}$ and $\mathrm{Gd}^{3+}$ chelates of DO3APyAm were formed by heating an excess of the relative lanthanide chloride with the deprotected ligand at $\mathrm{pH} \sim 5.5$ for a few days. Chelation of the $\mathrm{Eu}^{3+}$ chelate was confirmed with ${ }^{1} \mathrm{H}$ NMR: $400 \mathrm{MHz}, \mathrm{D}_{2} \mathrm{O}, 298 \mathrm{~K}$ : small broad peaks, including those at $\delta(\mathrm{ppm}) 17.2,15.0,10.1,7.8,7.4,6.8,-0.8,-1.9,-3.1$, $-3.7,-6.4,-6.8,-9.2,-11.0,-12.6,-13.4,-14.8,-15.9$ indicate chelate formation. These chelates were then purified by reverse phase preparative-HPLC, as described in Tables A.3 and A.4, and monitored at $268 \mathrm{~nm}$ for the pyridyl moiety; these purification processes resulted in lost product. 
Table A.3: Purification attempt of LnDO3APyAm chelates

\begin{tabular}{cccccc}
\hline Time (min) & $0-15$ & 20 & $23-28$ & $30-32$ & $34-40$ \\
\hline$\% \mathrm{H}_{2} \mathrm{O}(0.037 \% \mathrm{HCl})$ & 100 & 65 & 60 & 20 & 100 \\
$\% \mathrm{MeCN}$ & 0 & 35 & 40 & 80 & 0 \\
\hline
\end{tabular}

Table A.4: Purification attempt of LnDO3APyAm chelates

\begin{tabular}{ccccccc}
\hline Time $(\min )$ & 0 & 4 & 34 & 40 & 42 & $45-55$ \\
\hline$\% \mathrm{H}_{2} \mathrm{O}(0.037 \% \mathrm{HCl})$ & 100 & 80 & 65 & 60 & 20 & 100 \\
$\% \mathrm{MeCN}$ & 0 & 20 & 35 & 40 & 80 & 0 \\
\hline
\end{tabular}

